\title{
Diffusion in metallic glasses and supercooled melts
}

\author{
Franz Faupel* \\ Lehrstuhl für Materialverbunde, Technische Fakultät, Universität Kiel, D-24143 Kiel, \\ Germany \\ Werner Frank \\ Max-Planck-Institut für Metallforschung, D-70506 Stuttgart, Germany \\ and Institut für Theoretische und Angewandte Physik, Universität Stuttgart, \\ D-70550 Stuttgart, Germany \\ Michael-Peter Macht \\ Hahn-Meitner Institut, D-14109 Berlin, Germany \\ Helmut Mehrer \\ Institut für Materialphysik, ${ }^{\dagger}$ Universität Münster, D-48149 Münster, Germany \\ Volkmar Naundorf \\ Hahn-Meitner Institut, D-14109 Berlin, Germany \\ Klaus Rätzke \\ Lehrstuhl für Materialverbunde, Technische Fakultät, Universität Kiel, D-24143 Kiel, \\ Germany \\ Herbert R. Schober \\ Institut für Festkörperforschung, Forschungszentrum Jülich, D-52425 Jülich, Germany \\ Suman K. Sharma \\ Department of Physics, Malaviya National Institute of Technology, Jaipur 302 017, India \\ Helmar Teichler \\ Institut für Materialphysik, Universität Göttingen, D-37073 Göttingen, Germany \\ (Published 24 February 2003)
}

\begin{abstract}
Amorphous metallic alloys, also called metallic glasses, are of considerable technological importance. The metastability of these systems, which gives rise to various rearrangement processes at elevated temperatures, calls for an understanding of their diffusional behavior. From the fundamental point of view, these metallic glasses are the paradigm of dense random packing. Since the recent discovery of bulk metallic glasses it has become possible to measure atomic diffusion in the supercooled liquid state and to study the dynamics of the liquid-to-glass transition in metallic systems. In the present article the authors review experimental results and computer simulations on diffusion in metallic glasses and supercooled melts. They consider in detail the experimental techniques, the temperature dependence of diffusion, effects of structural relaxation, the atom-size dependence, the pressure dependence, the isotope effect, diffusion under irradiation, and molecular-dynamics simulations. It is shown that diffusion in metallic glasses is significantly different from diffusion in crystalline metals and involves thermally activated, highly collective atomic processes. These processes appear to be closely related to low-frequency excitations. Similar thermally activated collective processes were also found to mediate diffusion in the supercooled liquid state well above the caloric glass transition temperature. This strongly supports the mode-coupling scenario of the glass transition, which predicts an arrest of liquidlike flow already at a critical temperature well above the caloric glass transition temperature.
\end{abstract}

\section{CONTENTS}

I. Introduction

238

II. Experimental Techniques

240

\footnotetext{
*Corresponding author. Electronic address: ff@tf.uni-kiel.de ${ }^{\dagger}$ Formerly: Institut für Metallforschung
}

A. Radiotracer method 240

1. Mechanical sectioning 241

2. Sputter sectioning 241

B. Other profiling and detection methods 241

1. Secondary ion mass spectrometry 241

2. Auger electron spectroscopy 241

3. Rutherford backscattering spectrometry 241

4. Nuclear-reaction analysis 241 
C. Indirect methods

III. Structural Relaxation

A. Structural relaxation and the enthalpy (or volume) -versus-temperature diagram

B. Structural relaxation of diffusion properties

1. Conventional metallic glasses

2. Bulk metallic glasses

IV. Temperature Dependence of Diffusion
A. Introduction
B. Diffusion in the glassy state
C. Diffusion in the supercooled liquid state
D. Correlation between $D_{0}$ and $H$

V. Size Dependence
A. Remarks on atomic size
B. Solutes in crystalline metals
C. Solutes in amorphous alloys

VI. Pressure Dependence
A. Introduction
B. Data overview
C. Discussion
1. Systems with almost vanishing pressure dependence
2. Systems with considerable pressure dependence

VII. Isotope Effect
A. Motivation and definition
B. Diffusion in conventional metallic glasses
C. Diffusion in the deeply supercooled liquid state of bulk metallic glasses

VIII. Diffusion Under Irradiation

A. Theoretical considerations

1. Diffusion enhancement by radiationproduced mobile self-defects [mechanism (i)]

2. Diffusivity alterations via radiationinduced structural changes [mechanism (ii)]

B. Experiments and their interpretations

1. Diffusion under high-transferred-energy irradiation

2. Diffusion under low-transferred-energy irradiation

C. Facts and speculations

1. Defects in metallic glasses and the nature of the $\delta$ phase

2. Diffusion mechanisms in metallic glasses-a proposal stimulated by radiation-enhanceddiffusion results

D. Molecular-dynamics simulations of radiationdamage-induced diffusion

IX. Computer Simulations
A. Introductory remarks
B. Molecular-dynamics results concerning diffusion coefficients in melts and glasses
C. Motion in an amorphous solid
D. Motion in a liquid
E. Isotope effect
F. Activation volume
G. Heterogeneity
H. Reduced dimensionality

X. Summary and Concluding Remarks

Acknowledgments

List of Symbols

References
242

242

242

243

244

247

249

249

250

251

252

253

253

254

255

257

257

258

259

259

259

261

261

262

263

264

264

264

264

265

265

265

266

267

\section{INTRODUCTION}

During the past few years, diffusion in glasses and supercooled liquids has been a subject of considerable interest, particularly in the context of the interpretation of the liquid-to-glass transition as a kinetic phenomenon (Jäckle, 1986; Richter et al., 1989; Angell, 1995; Meyer et al., 1996; Greer, 1999; Tang et al., 1999). In so-called fragile glasses that undergo a pronounced glass transition in a narrow temperature range, such as most glassy polymers and amorphous metallic alloys, recent modecoupling theories predict a dynamical phase transition at a critical temperature $T_{c}$ well above the caloric glass transition temperature $T_{g}$ (Götze and Sjögren, 1991). Below $T_{c}$, liquidlike diffusion is predicted to freeze in, and long-range atomic transport is envisaged as a medium-assisted, highly collective hopping process, in which clusters of atoms perform thermally activated transitions into new configurations (Sjögren, 1991). Collective hopping has also been proposed to be closely related to the localized low-frequency excitations which appear to be a universal phenomenon occurring in all types of glasses with topological disorder (Suck and $\mathrm{Ru}$ din, 1983; Buchenau et al., 1986, 1988; Inoue et al., 1991; Schober et al., 1993).

Metallic glasses (also termed amorphous alloys throughout this article) are the paradigm of dense random packing and hence of great fundamental interest (Spaepen, 1981; Cahn, 1991). Until recently, however, it was not possible to study long-range atomic transport in the supercooled liquid state because of the strong tendency of conventional metallic glasses to crystallize when heated through the glass transition. This is why the discovery of multicomponent bulk metallic glasses that exhibit a much greater resistance to crystallization (Inoue et al., 1990a; Peker and Johnson, 1993) has triggered many investigations of diffusion in metallic glasses and their supercooled melts (Geyer et al., 1995, 1996; Budtke et al., 1997; Ehmler et al., 1998; Fielitz et al., 1999; Knorr, Macht, Freitag, and Mehrer, 1999; Knorr, Macht, and Mehrer, 1999; Nakajima et al., 1999; Rehmet et al., 2001, Zumkley et al., 2001).

Research on diffusion in metallic glasses has also been stimulated by the technological importance of these materials (Luborsky, 1983; Hilzinger, 1999). They can be designed to combine properties of ordinary glasses with the ductility of metals and may exhibit excellent softmagnetic properties or corrosion resistance. Moreover, the above-mentioned multicomponent metallic alloys, which can be produced at low cooling rates as bulk glasses, offer a host of new applications (Inoue, 1998; Johnson, 1999b). Since metallic glasses are nonequilibrium materials, they undergo various rearrangement processes, such as structural relaxations, phase separations, and crystallization, when exposed to elevated temperatures (Spaepen, 1981; Haasen, 1986; Cahn, 1991). Therefore the diffusion behavior of metallic glasses controls their thermal stability and plays a significant role in their production.

Despite the fundamental and technological interest in 
diffusion in metallic glasses and their supercooled melts, the subject is far from being understood. This is reflected by controversy and lively discussion in the literature. ${ }^{1}$ Because of similarities to the diffusion in crystalline metals, diffusion in metallic glasses has often been described by concepts borrowed from diffusion in crystals (Averback, 1991). Kinks in the Arrhenius plots at the caloric glass transition temperature have been attributed to a transition from single-atom jumps to viscous flow (Greer, 1999; Tang et al., 1999). By contrast, various recent experimental results (Buchenau et al., 1986, 1988; Scharwaechter et al., 1996a, 1996b; Frank, 1997; Ehmler et al., 1998; Meyer et al., 1998) and computer simulations (Hörner, 1993; Schober et al., 1993; Frank et al., 1996; Schober et al., 1997; Teichler, 1997, 2001; Rößler and Teichler, 2000) lend support to the view that diffusion in both the glassy and the deeply supercooled liquid state of metallic alloys is a highly collective hopping process.

This article aims at reviewing the recent progress in the field. Key points to be covered concern the role of single-atom jumps versus collective hopping and the question of whether there is a change in the diffusion mechanism at the caloric glass transition temperature $T_{g}$. Furthermore, the effect of excess free volume, quenched in by rapid cooling from the liquid state, on the diffusion behavior will be addressed.

Following this introduction, Sec. II deals with the experimental techniques available for diffusion studies in metallic glasses. Section III is devoted to irreversible structural relaxation (aging), which is accompanied by a drop in the diffusivity $D$, which finally approaches a plateau value allowing reproducible diffusion measurements to be carried out. Long-time relaxation near the caloric glass transition temperature from the glassy to the supercooled liquid state is also considered.

Section IV is concerned with the temperature dependence of diffusion. In seeming contrast to the presumption that in amorphous solids distributions of activation enthalpies exist, in the relaxed glassy state the temperature dependence of the diffusion coefficients are Arrhenius-like in the entire regime accessible by experiments, i.e., over many decades (Rank et al., 1990). For different radiotracer-amorphous-alloy combinations, the preexponential factors $D_{0}$ vary over more than 14 orders of magnitude, and the activation enthalpies $H$ also cover a very broad range (see, for example, Scharwaechter et al., 1996a). These findings point to a diffusion behavior that differs radically from that in crystalline materials. Arrhenius plots of diffusion coefficients in bulk glasses show a kink near the caloric glass transition temperature with a higher effective activation enthalpy above $T_{g}$. The kink temperature shifts to lower temperatures for slower diffusers.

\footnotetext{
${ }^{1}$ See, for example, Horváth, Pfahler, et al., 1985; Pfahler et al., 1985; Cantor, 1986; Horváth et al., 1987; Limoge and Bocquet, 1990; Averback, 1991; Faupel, 1992; Frank et al., 1994; Mehrer, 1996; Frank, 1997; Kronmüller, 1998; Geyer et al., 1999.
}

In Sec. V, data on the dependence of diffusion in metallic glasses on the size of the diffusants are presented. These provide important clues to the understanding of the underlying diffusion mechanism when compared to the diffusant-size dependencies for vacancy and interstitial mechanisms in crystalline metals.

The subject of Sec. VI is the pressure dependence of diffusion in metallic glasses. As conventionally done for thermally activated processes, the pressure dependence is expressed in terms of activation volumes. Depending on the alloys and the diffusers considered, activation volumes can almost vanish or be of the order of magnitude of the mean atomic volume, hinting at different diffusion mechanisms. (For a recent data review see Rätzke et al., 2001.) Section VI also includes first results on the pressure dependence of diffusion in a supercooled metallic melt (Knorr, Macht, Freitag, and Mehrer, 1999; Knorr, Macht, and Mehrer, 1999).

Isotope-effect measurements as key experiments to demonstrate collective diffusion are the subject of Sec. VII. The isotope effect reflects the dependence of the tracer diffusivity on the mass of the diffusing atom. By now, the isotope effect of Co diffusion has been determined in many metallic glasses with both small and large activation volumes. The very small isotope effects observed lend strong support to the view that in the glassy state diffusion occurs by collective hopping of a large number of atoms. (For a recent review see Faupel et al., 2001.) Recent measurements demonstrate that in the deeply supercooled state of bulk metallic glasses the isotope effects are also very small (Ehmler et al., 1998). Large isotope effects in an as-quenched metal-metalloid alloy are attributed to diffusion via quenched-in localized defects that are stable over several jumps (Rätzke et al., 1992). Such defects are denoted as quasivacancies throughout this article.

Valuable insight into the mechanisms of diffusion in metallic glasses also comes from investigations of diffusion under simultaneous particle irradiation (Sec. VIII). While early experiments (Bøttiger et al., 1987; Averback and Hahn, 1988; Tyagi et al., 1991a) involving large energy transfers to the matrix atoms showed an irradiation-induced decrease of the diffusion enthalpy $H$ as known from diffusion-under-irradiation studies on crystals, in recent experiments (Scharwaechter et al. 1996b; Schuler et al., 1997, 1999) with small energy transfers no irradiation effect was observed in relaxed metallic glasses with large $D_{0}$ values. By contrast, lowenergy transfers to relaxed metallic glasses with small $D_{0}$ values resulted in a pronounced increase of $D_{0}$ while the activation enthalpy $H$ remained unchanged. From this it was concluded that diffusion in relaxed metallic glasses with small $D_{0}$ values is confined to an unrelaxed network embedded in the otherwise relaxed amorphous matrix and that low-energy irradiation of these materials produces unrelaxed spots, giving rise to irradiationinduced diffusion enhancement.

Although quantities such as the activation enthalpy of diffusion, preexponential factor, activation volume, and isotope effect contain crucial microscopic information, 


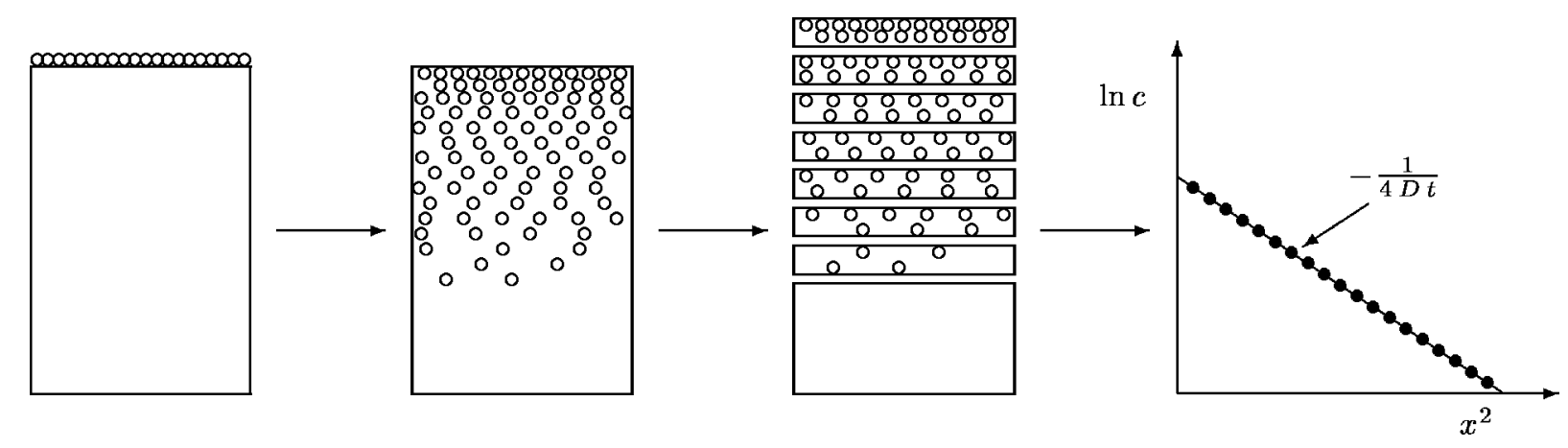

FIG. 1. Schematic illustration of the tracer method. The major steps (deposition of the tracer atoms, diffusion annealing, serial sectioning, and evaluation of the penetration profile) are indicated.

they do not yield a detailed picture of the atomic jump processes. This is why molecular-dynamics simulations (Sec. IX) are of paramount importance. After a short introduction to the simulation procedure, it will be shown that diffusive atomic displacements vary with the type and composition of the amorphous alloys. Though mainly collective, particularly chainlike displacements, are observed in certain structures, single-jump events also occur. Thermally activated collective hopping is seen in the deeply supercooled liquid state, too, while far above the glass transition temperature liquidlike viscous flow via atomic collisions prevails. Recent simulations of the isotope effect of diffusion reveal a transition from large isotope effects in the ordinary liquid state to very small ones in the supercooled state, similar to those observed by experiments in the glassy state. The connection between collective displacements and localized lowfrequency relaxations is a further topic to be discussed. Additionally the activation volume of diffusion under pressure, dynamical heterogeneity in the melt, and the consequences of reduced dimensionality are addressed.

Finally, Sec. X summarizes the results of the preceding sections and formulates the main conclusions. Emphasis is put on salient features of diffusion in metallic glasses and supercooled liquids, which are shown to be clearly different from those in crystalline metals. The available results provide strong evidence for the existence of highly collective hopping processes and their relation to glassy-state low-frequency excitations. In accordance with the predictions of the mode-coupling theory, neither diffusion measurements on long-time-relaxed bulk metallic glasses nor computer simulations show any indications of a change in the diffusion mechanism at the caloric glass transition temperature.

\section{EXPERIMENTAL TECHNIQUES}

The diffusion of atoms in an otherwise homogeneous solid can be described by Fick's second law,

$$
\frac{\partial C}{\partial t}=D \frac{\partial^{2} C}{\partial x^{2}},
$$

where $C(x, t)$ denotes the concentration of atoms at position $x$ and time $t$. If tiny amounts of tracer atoms are used, the diffusion coefficient $D$ is independent of con- centration. In a diffusion experiment, tracer atoms are usually tagged by their radioactivity, by their isotopic mass, or, in the case of foreign-atom diffusion, sometimes just by their chemical nature. In most experiments, at the beginning a thin layer of the diffusing tracers $\left(S_{0}\right.$ tracer atoms per unit area) is deposited on the surface $x=0$ of a sample. Then in a semi-infinite sample the concentration distribution after a diffusion anneal is given by

$$
C(x, t)=\frac{S_{0}}{\sqrt{\pi D t}} \exp \left(-\frac{x^{2}}{4 D t}\right) .
$$

Equation (2.2) is denoted as the thin-film solution (Gaussian solution) of Fick's second law. The quantity $\sqrt{D t}$ is a typical diffusion length, characteristic of diffusion problems. If the initial distribution of the diffusing atoms is more complicated, e.g., after implantation of the tracer atoms, a more sophisticated solution of Fick's second law must be used. Such solutions can be found, for example, in Crank (1975). The major task of a diffusion experiment is to study the concentration/depth profile and to deduce the diffusion coefficient by comparison with the appropriate solution of Fick's second law, e.g., Eq. (2.2).

\section{A. Radiotracer method}

Many of the most reliable diffusion studies on solids, including metallic glasses, have been performed by radiotracer techniques (see, for example, Rothman, 1984; Mehrer, 1990; Koiwa et al., 1993; Mehrer et al., 1997; Mehrer and Wenwer, 1998). Due to the high sensitivity of nuclear counting facilities, radiotracer studies are often superior to other techniques (see below). A very important advantage is that self-diffusion-the most basic diffusion process in solids - can be studied by radiotracers of matrix atoms in a straightforward manner.

Consider the experimental procedure in the tracer diffusion experiment shown schematically in Fig. 1. The tracer atoms are deposited on a polished, flat surface of the diffusion sample. Evaporation, dripping of a liquid solution, and electrodeposition of the radiotracer onto the surface are the major deposition techniques. Implantation of the radioisotopes sometimes offers advantages (Rothman, 1984; Mehrer, 1987). 
Following the tracer deposition, an isothermal diffusion anneal is performed at a temperature $T$ for a diffusion time $t$. The best way to determine the resulting concentration/depth profile is by serial sectioning of the sample and subsequent determination of the number of tracer atoms per section. Provided that the experimental conditions were chosen in such a way that the deposited layer is thin compared with the mean diffusion length, the distribution after the diffusion anneal is described by Eq. (2.2). If radioactive tracers are used, the specific activity per section is proportional to the tracer concentration. The specific activity is obtained from the section weight and the counting rate, which is conveniently determined by nuclear counting facilities such as $\gamma$ - or $\beta$-counting devices. According to Eq. (2.2), a plot of the logarithm of the specific activity versus the penetration distance squared is linear. Its slope equals $-(4 D t)^{-1}$. From the slope and the diffusion time the tracer diffusivity $D$ is obtained.

In the following, the serial sectioning techniques in use will be briefly described.

\section{Mechanical sectioning}

For diffusion lengths of at least several micrometers, mechanical techniques are applicable (for a review see Rothman, 1984). Lathes and microtomes are appropriate for ductile samples, grinding devices for brittle ones. However, these sectioning techniques are usually too crude to measure the fairly low diffusivities in metallic glasses reliably. Nevertheless, in an early experiment on such a material, mechanical sectioning was used (Schuehmacher and Guiraldenq, 1983).

\section{Sputter sectioning}

Diffusion studies in conventional metallic glasses are restricted to temperatures below the crystallization temperature and therefore require measurements of very small diffusivities. Such measurements are possible using sputtering techniques. Gupta et al. (1975) were probably the first who applied rf sputter sectioning to study diffusion in an amorphous alloy. Up-to-date devices for serial sectioning of radioactive diffusion samples by ion-beam sputtering are described, for example, by Faupel et al. (1992) and Wenwer et al. (1996). Sectioning of shallow diffusion zones, which correspond to diffusion lengths between several tens of $\mathrm{nm}$ and several $\mu \mathrm{m}$, is possible by employing ion-beam sputtering. Depth calibration can be performed by measuring the weight loss that occurs during the sputtering process or by determining the depth of the sputter crater, e.g., by interference microscopy or profileometer techniques. In the abovementioned devices, oblique incidence of the ion beam and low ion energies are used to minimize knock-on effects, and the sample is rotated to achieve a constant lateral sputtering rate. For a reasonable range of annealing times, a diffusivity range between about $10^{-24} \mathrm{~m}^{2} \mathrm{~s}^{-1}$ and $10^{-16} \mathrm{~m}^{2} \mathrm{~s}^{-1}$ can be examined.

\section{B. Other profiling and detection methods}

Several other profiling and detection methods have occasionally been used to measure concentration/depth profiles in amorphous alloys. We mention those used most frequently in what follows.

\section{Secondary ion mass spectrometry}

Reliable diffusion data for amorphous alloys have been obtained in combination with sputter depth profiling using secondary ion mass spectrometry (SIMS). This technique is mainly appropriate for diffusion studies of foreign atoms. It can also be used in self-diffusion measurements if enriched stable tracer isotopes are available. The concentration range of self-diffusion profiles is then limited by the natural abundance of the stable isotope in the material. This limitation was avoided in some SIMS studies of self-diffusion on amorphous Nicontaining alloys by using the radioisotope ${ }^{63} \mathrm{Ni}$ as tracer (Tyagi et al., 1990, 1991a).

\section{Auger electron spectroscopy}

Auger electron spectroscopy (AES) was used in combination with sputter profiling by Stelter and Lazarus (1987) to measure $\mathrm{Au}$ and $\mathrm{Ag}$ diffusion in amorphous $\mathrm{Cu}-\mathrm{Zr}$ and by Sharma and Mukhopadhyay (1990) in a study of $\mathrm{Cu}$ and $\mathrm{Al}$ diffusion in amorphous $\mathrm{Zr}_{61} \mathrm{Ni}_{39}$. Auger electron spectroscopy is only applicable to the diffusion of foreign atoms since it does not provide isotopic analyses.

\section{Rutherford backscattering spectrometry}

In Rutherford backscattering spectrometry experiments, a high-energy beam of monoenergetic $\alpha$ particles is used. These particles are preferentially scattered by heavy nuclei in the sample, and the energy spectrum of scattered $\alpha$ particles can be used to determine the concentration distribution of the scattering nuclei as a function of depth below the specimen surface. This technique is particularly suitable for detecting heavy elements in a matrix of substantially lower atomic weight. A detailed procedure for the Rutherford backscattering analysis of diffusion data for metallic glasses can be found in the papers of Sharma, Banerjee, et al. (1988) and Sharma, Kuldeep, and Jain (1988). Further examples are studies of $\mathrm{Au}$ diffusion in a $\mathrm{Pd}-\mathrm{Cu}-\mathrm{Si}$ metallic glass by Chen et al. (1978) and of Au diffusion in various glasses by Akhtar et al. (1982).

\section{Nuclear-reaction analysis}

High-energy particles can also be used to study diffusion of light elements if the nuclei can be made to undergo a suitable nuclear reaction. For example, Birac and Lesueur (1976) used a beam of high-energy neutrons and the nuclear reaction ${ }^{6} \mathrm{Li}+n \rightarrow{ }^{3} \mathrm{H}+\alpha$ to study Li diffusion in amorphous $\mathrm{Pd}_{80} \mathrm{Si}_{20}$. Kijek et al. (1986) measured the diffusion of ion-implanted boron in amorphous $\mathrm{Ni}_{59.5} \mathrm{Nb}_{40.5}$ by irradiating the amorphous alloy 
with high-energy protons, detecting the $\alpha$ particles emitted in the nuclear reaction ${ }^{11} \mathrm{~B}+p \rightarrow{ }^{8} \mathrm{~B}+\alpha$, and determining the concentration profile of ${ }^{11} \mathrm{~B}$ from the number and energy of the emitted $\alpha$ particles as a function of the incident-proton energy.

Rutherford backscattering spectrometry and nuclearreaction analysis require a depth calibration, which is not always based on very accurate data of the stopping power in the matrix for those particles emitted by the nuclear reaction. The depth resolution is also usually inferior to that achievable in careful radiotracer and SIMS profiling studies.

\section{Indirect methods}

Several indirect methods have also been used to investigate diffusion in amorphous alloys. Some researchers (e.g., Köster and Herold, 1980; Köster et al., 1981; Tiwari et al., 1981) estimated self-diffusion coefficients in iron-based amorphous alloys from crystal growth rates during crystallization, by assuming that the kinetics of crystal growth is diffusion controlled. Indirect estimates of self-diffusion coefficients should be treated with caution. Usually, the mechanism and kinetics of a process are established from known diffusion data rather than vice versa.

An elegant technique, also sensitive to very small diffusivities, was used by Rosenblum et al. (1980) and Greer et al. (1982). Diffusion in compositionally modulated amorphous multilayer specimens was monitored via the intensity decay of the small-angle x-ray peak corresponding to the interlayer separation. Such experiments yield interdiffusion coefficients which pertain to diffusion in a chemically inhomogeneous material. The interpretation of interdiffusion requires additional knowledge of thermodynamic factors, which is usually not available for amorphous alloys. By contrast, tracer diffusion pertains to homogeneous alloys, since the tiny amounts of tracer atoms, especially in radiotracer studies, will not significantly alter the composition.

\section{STRUCTURAL RELAXATION}

\section{A. Structural relaxation and the enthalpy (or volume) -versus-temperature diagram}

The glass-formation behavior of any material is conveniently discussed on the basis of either an enthalpy or a volume-versus-temperature diagram like that shown in Fig. 2 (see, for example, Cahn, 1983; Davies, 1983; Luborsky, 1983; Shelby, 1997). Since enthalpy and volume behave in a similar fashion, the choice of the ordinate is somewhat arbitrary. Let us envisage a small volume of a melt at a temperature well above the melting temperature $T_{m}$ of the material. With decreasing temperature, the atomic structure of the melt will gradually change and will be characteristic of the temperature at which the melt is held. Cooling down below $T_{m}$ will convert the melt to the crystalline state provided that the kinetics permits nucleation of the crystalline phase. If this

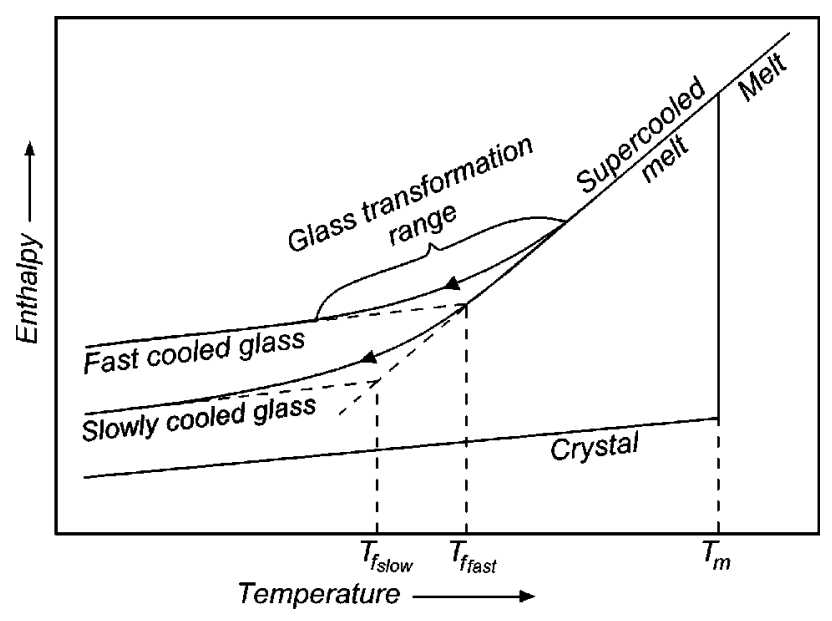

FIG. 2. The effect of temperature on the enthalpy (volume) of a glass-forming material.

occurs, the enthalpy (or volume) will decrease abruptly to the value typical of the crystal. Continued cooling of the crystal will result in a further decrease of enthalpy (volume) due to the specific heat (or thermal contraction) of the crystal.

If the melt can be cooled below $T_{m}$ without crystallization, a supercooled melt is obtained, which, of course, is metastable with respect to crystallization. The formation of crystalline nuclei takes time. If the cooling rate is sufficiently high, nucleus formation does not occur. Upon further cooling, the structure of the liquid continues to remain in metastable configurational equilibrium as the temperature decreases, but no abrupt change occurs in enthalpy (volume) due to a phase transformation. With increasing supercooling, the viscosity between $T_{m}$ and $T_{f}$ (see below) increases by about 15 orders of magnitude. The structure of the supercooled melt begins to deviate from that which would be present if sufficient time were allowed to reach configurational equilibrium. This situation usually occurs for viscosities around $10^{12} \mathrm{Pas}\left(=10^{13}\right.$ Poise). Correspondingly the enthalpy (volume) begins to deviate from the equilibrium line, following a line of gradually decreasing slope (Fig. 2) until it becomes determined by the specific heat (thermal expansion) of the "isoconfigurational melt" when the viscosity becomes so high that the structure of the material becomes fixed and is no longer temperature dependent. The "mobile melt" has now become a rigid glass. A glass can be defined as a supercooled melt congealed to a rigid, isoconfigurational state. The temperature region lying between the limits where the enthalpy (volume) is either that of an equilibrated liquid or that of a glass is denoted as the glass transformation region.

Since the glass transformation region is controlled by kinetic factors, i.e., by the viscosity of the supercooled melt, a slower cooling rate will allow the enthalpy (volume) to follow the equilibrium line to lower temperatures. The glass obtained will have a lower enthalpy (volume) than that obtained at the faster cooling rate. The atomic arrangement will be that characteristic of the supercooled melt at lower temperatures. Although 


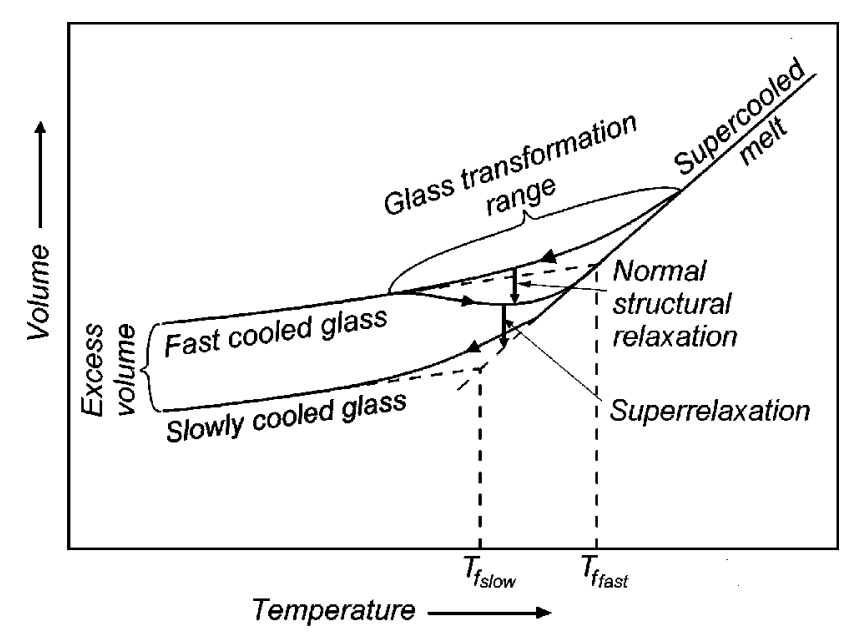

FIG. 3. Illustration of structural relaxation in the volume (enthalpy) diagram of a glass-forming material.

the glass transformation occurs over a temperature range, it is convenient to define a temperature that allows us to express the difference in thermal history between glasses. The extrapolations of the supercooled melt and glass lines intersect at a temperature which in the literature on glasses is called the fictive temperature $T_{f}$. A glass produced at a slower cooling rate has a lower $T_{f}$.

Finally, it is convenient to introduce the concept of the glass transition temperature or glass transformation temperature $T_{g}$. The glass transition temperature is used as an indication of the onset of the glass transformation during heating of a glass. This temperature is frequently determined by changes in thermal analysis curves (caloric glass transition temperature) or in thermal expansion curves. $T_{g}$ values obtained from these two methods are similar although not identical. In addition, $T_{g}$ is a function of the heating rate used during the analysis. Although the glass transition temperature and the fictive temperature are not identical, the differences are small, usually not more than a few $\mathrm{K}$. Therefore $T_{g}$ is a useful indicator of the approximate temperature where the supercooled melt converts to a glass.

Glasses and supercooled melts are thermodynamically metastable in a twofold sense: (i) Both can undergo crystallization during which the material transforms to one or more crystalline phases. This crystallization transformation is not the subject of this paper. (ii) It is obvious that the properties of a glass may depend on the thermal history of the particular sample measured. When a glass is reheated to the glass transformation range, its properties may change due to a process called structural relaxation.

Structural relaxation of an amorphous material leads from a less stable to a more stable-but still metastable-amorphous state. It is accompanied by a number of changes in physical properties. Changes due to structural relaxation are conveniently explained by considering the volume-versus-temperature diagram (Fig. 3). The volume can be altered by heat treatment at a specific temperature for times sufficient to allow

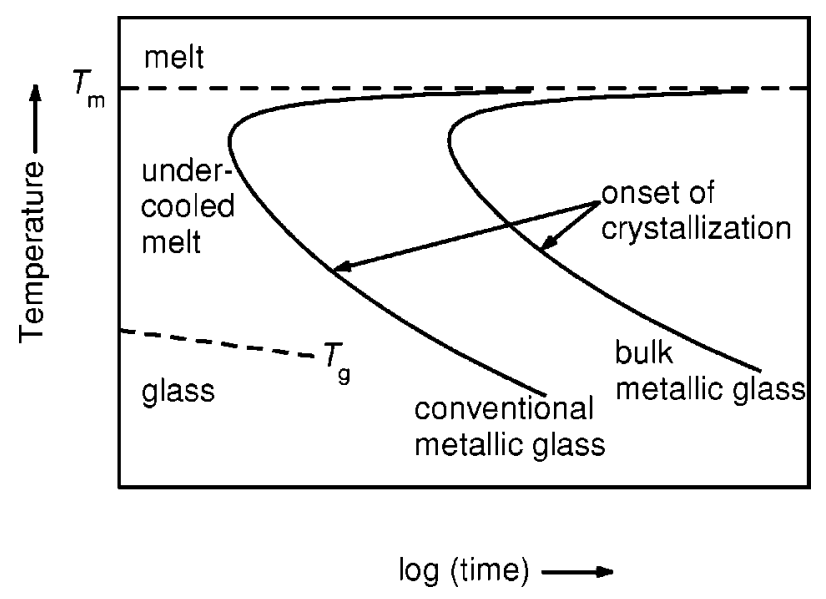

FIG. 4. Schematic time-temperature transformation diagram comparing the crystallization kinetics of conventional and bulk glass-forming alloys.

equilibration of the structure to a volume appropriate for the treatment temperature. A fast-cooled glass has a high fictive temperature, a large volume, and a low density. The volume difference is sometimes denoted as excess volume. If we reheat such a material to a temperature within the transformation range but below the original fictive temperature, the sample will readjust to the structure appropriate for the new temperature. Its volume will decrease. Although the changes in density occurring during structural relaxation are not particularly large (typically less than $1 \%$ ), they can be important for viscosity and ductility as well as for magnetic, elastic, electric, and diffusion properties. An early review on the effects of structural relaxation on various properties of metallic glasses was that of Chen (1983). In the following section, we concentrate on diffusion properties. Clearly, the extent of property changes for a given material will depend on its thermal history and hence on the way the glass was produced.

Structural relaxation is a phenomenon common to all kinds of glasses, including the so-called network glasses such as silicate, borate, or germanate glasses and the organic glasses. Structural relaxation effects in the field of organic glasses are denoted as physical aging effects. For a very recent example of structural relaxation effects in a nonmetallic glass we refer the reader to the papers of Schoo et al. $(2000,2001)$ and further literature discussed therein. Schoo et al. report on a decrease of ${ }^{22} \mathrm{Na}$ diffusion in sodium-borate glass with increasing annealing time. During annealing at $573 \mathrm{~K}$, the diffusivity decreases within $180 \mathrm{~h}$ by about $30 \%$. This behavior is similar to that of conventional metallic glasses discussed below.

\section{B. Structural relaxation of diffusion properties}

To put the development of metallic-glass-forming alloys in perspective, it is common practice to consider the time-temperature transformation (TTT) diagram. Figure 4 shows a schematic TTT diagram for crystallization of 
undercooled melts. At the thermodynamic melting temperature $T_{m}$ the time necessary for crystallization tends to infinity. With increasing undercooling the crystallization occurs at shorter times because the nucleation rate of the crystalline phase increases. At large undercoolings the crystallization rate decreases again because atomic transport in the undercooled melt becomes very sluggish. As a consequence, for intermediate undercooling temperatures the crystallization curve forms a "nose." A glass can be formed if the cooling is so fast that the crystallization nose can be avoided. Below the glass transition region around $T_{g}$ the undercooled melt becomes a glass.

Metallic glasses are usually obtained by cooling an alloy melt fast enough through the glass transition. Duwez and co-workers (Clement et al., 1960) were the first to produce a metallic glass. They reported glass formation by rapid cooling of an Au-Si alloy melt. For most metallic glasses, cooling rates of about $10^{6} \mathrm{~K} \mathrm{~s}^{-1}$ are necessary. We call such metallic glasses conventional metallic glasses. In the following decades, continuous casting processes like melt spinning were developed for laboratory and commercial manufacture of metallic glass ribbons and sheets. For conventional metallic glasses, the nucleation kinetics of the undercooled melt for crystallization is such that the time scale is in the 0.1-1 ms range at the nose of the nucleation curve of the time-temperature transformation diagram (TTT diagram) for crystallization.

Starting around 1990, the field of so-called bulk metallic glasses gained momentum. Bulk metallic glasses produce TTT diagrams with a crystallization nose in the range 1-100 s or more. Some multicomponent alloys (Sec. III.B.2) have an exceptional glass-forming ability comparable to that of silicate glasses. This also implies that they form an undercooled melt that is relatively stable and thus suitable for diffusion studies. By contrast, conventional metallic glasses undergo crystallization below the glass transition temperature and thus can be studied only below this temperature.

In Sec. III.B.1 we consider the effects of structural relaxation on diffusion in conventional metallic glasses. Structural relaxation in bulk metallic glasses is discussed in Sec. III.B.2. Bulk metallic glasses can relax to their supercooled liquid state-a process that may be denoted as super-relaxation (or long-time relaxation; Fig. 3). This process cannot occur in conventional metallic glasses. It is prevented by the onset of crystallization.

\section{Conventional metallic glasses}

In the very first diffusion experiment on a conventional metallic glass by Gupta et al. (1975) the authors did not consider the effect of structural relaxation. However, Chen (1974), probably the second group of researchers to measure diffusivity in a meltspun metallic glass using Rutherford backscattering profiling, reported that the diffusivity of $\mathrm{Au}$ in a $\mathrm{Pd}-\mathrm{Cu}-\mathrm{Si}$ metallic glass was reduced by progressive annealing under conditions that did not suffice to initiate crystallization. Interdiffu- sion and structural relaxation in compositionally modulated amorphous films produced by sputtering were studied by monitoring the satellites of x-ray scattering peaks during isothermal anneals by Rosenblum et al. (1980).

Unfortunately, in some of the earlier studies on diffusion in metallic glasses (reviewed by Cantor and Cahn, 1983; Limoge et al., 1983; Cantor, 1985), the authors did not always pay attention to structural relaxation effects. Often diffusion coefficients with low precision-no higher than half-an-order-of-magnitude accuracy-were obtained, which is insufficient to study structural relaxation in a systematic way. Moreover, in some of the earlier investigations structural relaxation was either overlooked or not taken into account. On the other hand, Cantor and Cahn (1983) had already mentioned that the diffusivity in as-quenched metallic glasses might drop during annealing due to structural relaxation. They pointed out that different diffusivities reported for the same material might be attributed to different methods of preparation and/or different states of structural relaxation.

Starting from the mid 1980s, the effect of structural relaxation on the diffusivity of as-quenched glasses was systematically studied by a number of researchers using either radiotracer techniques in combination with sputter sectioning or SIMS profiling. A review on diffusion in amorphous alloys which summarizes the effects of structural relaxation on diffusion for the first time in detail is that of Mehrer and Dörner (1989). In this context we also refer the reader to a data collection on diffusion in metallic glasses by Horváth (1990), in which diffusion studies with and without preannealing treatments are documented up to 1990.

A typical example of structural relaxation effects is shown in Fig. 5, which displays diffusion profiles of ${ }^{59} \mathrm{Fe}$ in as-quenched $\mathrm{Fe}_{40} \mathrm{Ni}_{40} \mathrm{~B}_{20}$ metallic glass after various annealing times $t$ from the work of Horváth, Freitag, and Mehrer (1985). Each curve corresponds to a single sample annealed for the indicated duration. The logarithm of the specific activity of ${ }^{59} \mathrm{Fe}$ is plotted versus the penetration distance $x$ squared and normalized to the annealing time $t$. The straight lines indicate that each profile matches the thin-film solution of Fick's second law given by Eq. (2.2). Their slope is equal to $-1 / 4 \bar{D}$. Obviously the diffusion coefficients depend on time, since otherwise all slopes in Fig. 5 should be the same. Time-averaged diffusivities $\bar{D}$ were obtained from Fig. 5 and from similar experiments at a slightly higher temperature. They are displayed in Fig. 6. The timeaveraged diffusivities decrease by about half an order of magnitude with increasing annealing time.

Similar effects of structural relaxation were reported by Horváth and Mehrer (1986) on ${ }^{59} \mathrm{Fe}$ and ${ }^{32} \mathrm{P}$ diffusion in $\mathrm{Fe}_{40} \mathrm{Ni}_{40} \mathrm{~B}_{20}$, by Pfahler et al. (1985) on ${ }^{59} \mathrm{Fe}$ diffusion in $\mathrm{Fe}_{41} \mathrm{Ni}_{41} \mathrm{~B}_{18}$, by Tyagi et al. (1990) on ${ }^{63} \mathrm{Ni}$ diffusion in $\mathrm{Fe}_{40} \mathrm{Ni}_{38} \mathrm{Mo}_{4} \mathrm{~B}_{18}$ and in $\mathrm{Fe}_{40} \mathrm{Ni}_{40} \mathrm{~B}_{20}$, by Horváth et al. (1988) on ${ }^{59} \mathrm{Fe}$ and ${ }^{95} \mathrm{Zr}$ diffusion in $\mathrm{Fe}_{x} \mathrm{Zr}_{100-x}$ glasses and on ${ }^{59} \mathrm{Fe},{ }^{95} \mathrm{Zr}$, and ${ }^{32} \mathrm{P}$ diffusion in various metalmetalloid and metal-metal amorphous alloys, by Dörner 


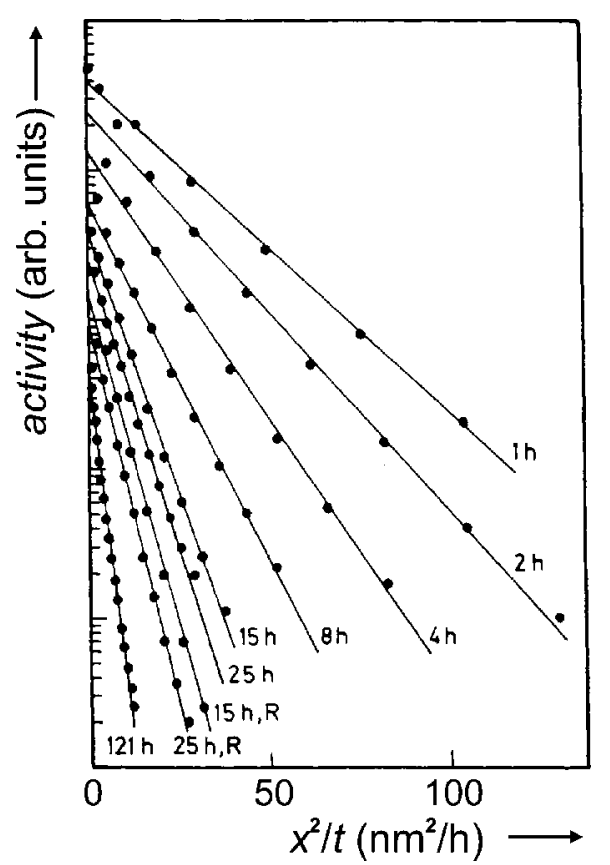

FIG. 5. Diffusion profiles of ${ }^{59} \mathrm{Fe}$ in as-quenched $\mathrm{Fe}_{40} \mathrm{Ni}_{40} \mathrm{~B}_{20}$ metallic glass measured after various annealing times at $593 \mathrm{~K}$. Each curve corresponds to a single sample annealed for the duration indicated. $R$ in the figure means that the sample was given a relaxation annealing treatment before the diffusion experiment was performed. From Horváth, Pfahler, et al., 1985.

and Mehrer (1991) on ${ }^{57} \mathrm{Co}$ in melt-spun $\mathrm{Co}_{89} \mathrm{Zr}_{11}$, by Rätzke et al. (1992) on ${ }^{57} \mathrm{Co}$ diffusion in $\mathrm{Co}_{76.7} \mathrm{Fe}_{2} \mathrm{Nb}_{14.3} \mathrm{~B}_{7}$, by Scharwaechter et al. (1996a) on ${ }^{59} \mathrm{Fe}$ diffusion in $\mathrm{Co}_{58} \mathrm{Fe}_{5} \mathrm{Ni}_{10} \mathrm{Si}_{11} \mathrm{~B}_{6}$ (see also Frank, 1997), by Zöllmer et al. (2000) on diffusion of ${ }^{57} \mathrm{Co} /{ }^{60} \mathrm{Co}$ in thin amorphous layers of $\mathrm{Co}_{51} \mathrm{Zr}_{49}$, and by Heesemann, Zöllmer, et al. (2000) on diffusion of ${ }^{57} \mathrm{Co} /{ }^{60} \mathrm{Co}$ in amorphous $\mathrm{Co}_{81} \mathrm{Zr}_{19}$ films. Small effects of relaxation treatments on the diffusivities of $\mathrm{Sb}, \mathrm{Au}$, and $\mathrm{Pb}$ in amorphous $\mathrm{Fe}_{82} \mathrm{~B}_{18}$, though not systematically investi-

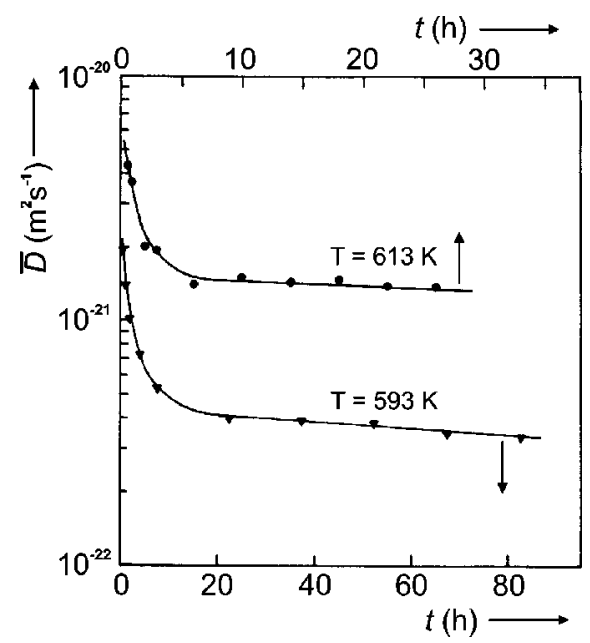

FIG. 6. Time-averaged diffusion coefficients of ${ }^{59} \mathrm{Fe}$ in $\mathrm{Fe}_{40} \mathrm{Ni}_{40} \mathrm{~B}_{20}$ for various annealing times at 593 and $613 \mathrm{~K}$. From Horváth, Pfahler, et al., 1985.

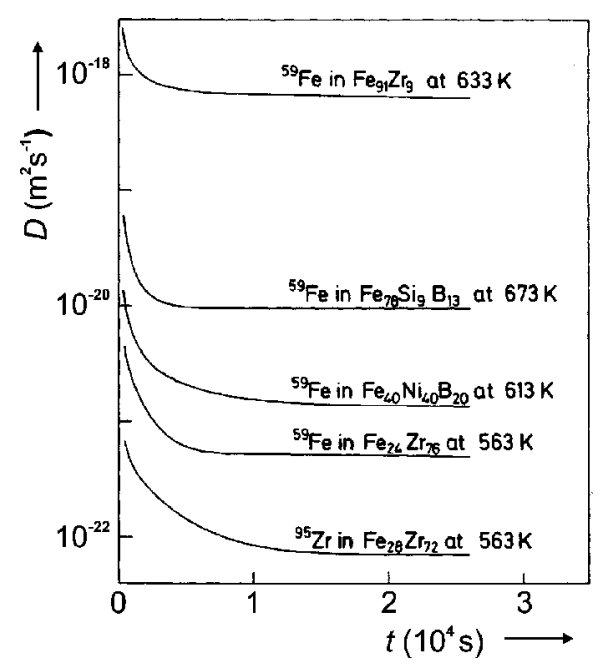

FIG. 7. Instantaneous diffusion coefficients in metallic glasses as functions of the annealing time (Horváth, Pfahler, et al., 1987; see also Frank, 1997).

gated, were reported by Sharma, Banerjee, et al. (1988), and Sharma, Kuldeep, and Jain (1988), who employed Rutherford backscattering spectrometric profiling.

If structural relaxation occurs during the diffusion annealing heat treatment, the diffusivity $D$ depends on time. If this is the case, Eq. (2.2) remains valid if $D$ is replaced by its time average,

$$
\bar{D}(t)=\frac{1}{t} \int_{0}^{t} D\left(t^{\prime}\right) d t^{\prime} .
$$

Equation (3.1) can be verified by showing that the thinfilm solution with the time-averaged diffusivity $\bar{D}$ is a solution of Fick's second law (2.1) with the timedependent diffusivity given by Eq. (3.2). Only the timeaveraged diffusivity $\bar{D}$ is accessible in a diffusion experiment. Of course, it will depend on time as well if structural relaxation occurs. Using Eq. (3.1) we get for the instantaneous diffusivity

$$
D(t)=\bar{D}(t)+t \frac{d \bar{D}(t)}{d t} .
$$

If enough values of the time-averaged $\bar{D}(t)$ are measured as a function of annealing time, the instantaneous diffusion coefficient can be calculated using Eq. (3.2). Figure 7 shows instantaneous diffusion coefficients for various as-quenched metallic glasses according to Horváth et al. (1987). The main feature of Fig. 7 is the continuous decrease of $D(t)$ to a plateau value, at least within experimental accuracy and on the time scale of the experiment (see also Frank, 1997). In the following, this plateau value is denoted as $D_{R}$. This diffusion coefficient pertains to the relaxed amorphous state. Temperature cycling confirms that a steady (relaxed) configuration is reached (Frank et al., 1988; Kronmüller and Frank, 1989).

A common feature of these studies is that the diffusivities of tracer atoms in as-quenched samples decrease 
during thermal annealing as a result of structural relaxations of the amorphous state. This effect may be described by the relationship

$$
D(t, T)=D_{R}(T)+\Delta D(t, T) .
$$

The diffusivity enhancement $\Delta D(t, T)$ in the unrelaxed state drops to zero upon sufficient annealing. The diffusivity $D_{R}(T)$ in the relaxed state depends on temperature only. Usually within the limits of experimental error it can be described by an Arrhenius equation [see Sec. IV, Eq. (4.1)].

The diffusivity enhancement $\Delta D$ in conventional metallic glasses is correlated with the excess volume present in the as-quenched material (Fig. 3). This excess volume anneals out during structural relaxation. It is interesting to note that structural relaxation is accompanied by an increase in density, which, for example, amounts to about $0.8 \%$ upon heating as-quenched ribbons of $\mathrm{Fe}_{40} \mathrm{Ni}_{40} \mathrm{~B}_{20}$ to about $400^{\circ} \mathrm{C}$ (Gerling et al., 1985). Positron annihilation studies have been frequently used to study structural relaxation (see, for example, the early review of Chen, 1983). Mihara et al. (1981) attribute changes in the Doppler-broadened line shape observed upon annealing $\mathrm{Fe}_{40} \mathrm{Ni}_{40} \mathrm{P}_{14} \mathrm{~B}_{6}$ below the crystallization temperature to the loss of excess volume of the as-quenched sample.

Applying a very general reasoning, we expect that atoms should require less energy to move through a more open (less dense) structure than to move through a less open (more dense) structure. In other words, the diffusivity of atoms in a fast-quenched glass should decrease during an annealing treatment at a temperature below the fictive temperature of the as-quenched glass. Sometimes the excess volume is also said to be due to quasivacancies, envisaged as localized defects that are stable over several jumps (Frank et al., 1988). In the language of quenched-in quasivacancies, the latter are mobile during structural relaxation and contribute to atomic diffusion. In contrast to self-diffusion in crystalline metals, which occurs via vacancies present in thermal equilibrium, the quasivacancies in an as-quenched amorphous alloy are present in supersaturation and hence anneal out when they become mobile. As a result, the diffusivities slow down until they reach their relaxed-state values.

The diffusivity enhancements illustrated above depend on the material, on its thermal history, and on the technique of glass production. For a given material, the diffusivity enhancement may be insignificant for samples with low fictive temperature. Indeed, some researchers have failed to observe a diffusivity reduction despite employing sensitive experimental techniques. For example, in the work of Hoshino et al. (1988) and of Hahn et al. (1988), amorphous layers of $\mathrm{Ni}-\mathrm{Zr}$ were produced by co-evaporation of $\mathrm{Co}$ and $\mathrm{Zr}$, and the diffusion broadening of buried layers of ${ }^{60} \mathrm{Co},{ }^{63} \mathrm{Ni}, \mathrm{Cu}, \mathrm{Fe}, \mathrm{Ti}$, and $\mathrm{Au}$ was studied using ion-beam sputtering for radiotracers, SIMS for light tracers ( $\mathrm{Cu}, \mathrm{Fe}, \mathrm{Ti})$, and Rutherford backscattering spectrometry for the heavy tracer $\mathrm{Au}$. No structural relaxation effects were observed. The authors attributed this to the fact that the evaporation technique produced well-relaxed samples. In their study of $\mathrm{Cu}$ and $\mathrm{Al}$ diffusion in the melt-spun $\mathrm{Ni}_{39} \mathrm{Zr}_{61}$ glass, Sharma and Mukhopadhyay (1990) did not find significant structural relaxation effects either.

Apparently conflicting results on the number of structural relaxation effects are likely due to different techniques of amorphous alloy preparation such as meltspinning, splat cooling, or co-evaporation. According to Fig. 3 different fictive temperatures lead to differences in the amount of structural relaxation. Different materials may also have differing susceptibilities to structural relaxation effects.

Effects of structural relaxation have also been found in measurements of the isotope effect of diffusion. Although isotope effects will be discussed in detail in Sec. VII, we mention here a study of Co diffusion and its isotope effect in melt-spun amorphous $\mathrm{Co}_{76.7} \mathrm{Fe}_{2} \mathrm{Nb}_{14.3} \mathrm{~B}_{7}$ by Faupel et al. $(1992,1993)$. The authors reported that Co diffusion decreased in the manner discussed above. As the Co diffusivity decreased, the isotope-effect parameter $E$ at different stages of structural relaxation showed a strong decrease from $E>0.5$ in the as-quenched state to $E=0.1$ in the relaxed state. The low isotope effect in relaxed material suggests that diffusion in structurally relaxed metallic glass is a collective process involving the simultaneous motion of several (about 10) atoms. The large isotope effect in the as-quenched material indicates the prevalence of singleatom jumps resembling the vacancy-mediated diffusion mechanism in crystalline metals.

By contrast, isotope-effect measurements of Co diffusion on thin amorphous $\mathrm{Co}_{51} \mathrm{Zr}_{49}$ sputter-deposited layers by Zöllmer et al. (2000) during structural relaxation show no change in the isotope effect. The low value of $E=0.01$ in the relaxed and in the as-quenched state suggests collective diffusion in both states in spite of the associated drop in diffusivity by about a factor of 3 . Similar results were obtained for Co diffusion in sputterdeposited $\mathrm{Co}_{81} \mathrm{Zr}_{19}$ layers (Heesemann et al., 2001). Small values of the isotope effect of Co diffusion in $\mathrm{Co}-\mathrm{Zr}$ glasses appear to be independent of composition and the degree of relaxation.

The effects of structural relaxation on Co diffusivity and the Co isotope effect for melt-spun amorphous $\mathrm{Co}_{76.7} \mathrm{Fe}_{2} \mathrm{Nb}_{14.3} \mathrm{~B}_{7}$ and for sputter-deposited layers of amorphous $\mathrm{Co}-\mathrm{Zr}$ layers are clearly different. For the melt-spun material, both diffusivity and the isotope effect decrease during structural relaxation. This decrease can be attributed to the annealing-out of quasivacancies, which are present in the as-quenched material. When structural relaxation is finished, only the collective contributions to diffusion are left. For sputter-deposited $\mathrm{Co}-\mathrm{Zr}$ films, the low isotope-effect parameter is not affected by structural relaxation, suggesting that collective diffusion dominates in the as-prepared and in the relaxed states. In this case, the origin of the drop in the diffusivity must be different from that in melt-spun material and cannot be attributed to the annealing-out of quasivacancies. An idea of Egami et al. (1980) provides 
a possible explanation. He proposed that local regions of higher and lower density (denoted as $n$ and $p$ defects) annihilate each other. Due to the anharmonicity of the interatomic potentials, this gives rise to an increase in density and hence to a decrease in diffusivity. Similar ideas are discussed in Sec. VIII.

\section{Bulk metallic glasses}

The history of bulk metallic glasses probably started with the work of Chen (1974), then at Bell Laboratories, who succeeded in forming millimeter-diameter rods of ternary $\mathrm{Pd}-\mathrm{Cu}-\mathrm{Si}$ alloys by suction casting methods at cooling rates of about $10^{3} \mathrm{~K} / \mathrm{s}$. Perhaps these ternary alloys were the first examples of bulk metallic glasses. In the early 1980s, Turnbull and co-workers (Drehmann et al., 1982; Kui et al., 1984) carried out experiments on Pd-Ni-P alloy melts and were able to demonstrate that these alloys form bulk-metallic-glass ingots of centimeter size at cooling rates of only $10 \mathrm{~K} / \mathrm{s}$.

Around 1990 the field of bulk metallic glasses developed rapidly when Inoue and co-workers in Sendai succeeded in producing amorphous aluminum alloys. They found exceptional glass-forming ability in rare-earth-rich alloys such as $\mathrm{La}-\mathrm{Al}-\mathrm{Ni}$ and $\mathrm{La}-\mathrm{Al}-\mathrm{Cu}$ (Inoue et al., 1990a). Glassy rods and bars with casting thicknesses of several millimeters were obtained. Studying similar quaternary and quinary alloys, the Inoue group developed alloys (e.g., La-Al-Cu-Ni) that form glasses at cooling rates lower than $100 \mathrm{~K} / \mathrm{s}$ and critical casting thicknesses up to $1 \mathrm{~cm}$ (Inoue et al., 1992). A similar family of $\mathrm{Mg}$ based alloys (e.g., $\mathrm{Mg}-\mathrm{Y}-\mathrm{Cu}, \mathrm{Mg}-\mathrm{Y}-\mathrm{Ni}$; Inoue, 1995) and a family of $\mathrm{Zr}$-based alloys (e.g., $\mathrm{Zr}-\mathrm{Cu}-\mathrm{Ni}-\mathrm{Al}$; Inoue et al., 1990b) were also developed.

These multicomponent glass-forming alloys demonstrated that bulk glass formation was a fairly ubiquitous phenomenon. Building on the work of Inoue's group, Johnson and co-workers at the California Institute of Technology (Peker and Johnson, 1993) developed a family of glass formers built on higher-order alloys of $\mathrm{Zr}$, Ti, $\mathrm{Cu}, \mathrm{Ni}$, and Be. Their glass-forming ability is comparable to that of silicate glasses. Bulk metallic glasses could now be produced by conventional metallurgical casting methods.

As already mentioned in the Introduction, the discovery of the bulk metallic glasses has triggered many investigations of diffusion in these glasses and their supercooled melts. Before this discovery it was not possible to study diffusion of metallic glasses in the strongly supercooled liquid state because of the strong tendency of conventional metallic glasses to crystallize when heattreated. When conventional metallic glasses are annealed, crystallization occurs before the glass transition temperature $T_{g}$ is reached. By contrast, bulk metallic glasses have crystallization temperatures $T_{x}$ that are higher than $T_{g}$ and therefore can be annealed at least for some time in the supercooled liquid state.

The alloy that has attracted perhaps the greatest interest from the viewpoint of diffusion is the five-component alloy $\mathrm{Zr}_{46.75} \mathrm{Ti}_{8.25} \mathrm{Cu}_{7.5} \mathrm{Ni}_{10} \mathrm{Be}_{27.5}$ (also known as Vitreloy
4), from which centimeter-size rods can be produced by casting techniques (see, for example, Johnson, 1999a). This alloy is particularly suited for diffusion experiments since its time-temperature transformation diagram in the range of the glass transition and crystallization is known from the work of Busch and Johnson (1998). In addition, Vitreloy 4 does not show any sign of decomposition in the temperature range of a supercooled liquid, whereas other bulk glass formers of the $\mathrm{Zr}-\mathrm{Ti}-\mathrm{Cu}-\mathrm{Ni}-\mathrm{Be}$ family-e.g., $\mathrm{Zr}_{41} \mathrm{Ti}_{14} \mathrm{Cu}_{12.5} \mathrm{Ni}_{10} \mathrm{Be}_{22.5}$, called Vitreloy 1-undergo spinoidal decomposition into two amorphous phases. According to Wiedenmann et al. (1996) and Schneider et al. (1997), Vitreloy 1 decomposes around $623 \mathrm{~K}$ within a few hours. In contrast to Vitreloy 1, the composition of Vitreloy 4 lies outside the miscibility gap.

The temperature dependence of diffusion in Vitreloy 4 has been measured for a variety of elements, including Be (Rehmet et al., 2001), B (Fielitz et al., 1999), Fe (Fielitz et al., 1999), Ni (Knorr, Macht, et al., 1999, 2000), Co (Ehmler et al., 1999; Fielitz et al., 1999), Al (Budke et al., 1997), Zr (Knorr et al., 2000), and Hf (Zumkley et al., 2001). In addition, interdiffusion of Be in Be-Vitreloy 4 diffusion couples was studied by Geyer et al. (1996). The results are collected in Fig. 8. An important observation is that the diffusivities of several elements exhibit a "nonlinear" Arrhenius behavior, i.e., the effective activation enthalpy $-k_{B} \partial \ln D / \partial T^{-1}$ and preexponential factor above the glass transition temperature $T_{g}$ are higher than below $T_{g}$. As a consequence, diffusivity in the glassy state is higher than expected from a lowtemperature extrapolation of the Arrhenius line that pertains to the supercooled liquid state. A similar break in the Arrhenius diagram was reported for other bulk metallic glasses. Examples are the studies of Knorr, Macht, and Mehrer (1999) on $\mathrm{Ni}$ diffusion in $\mathrm{Zr}_{65} \mathrm{Cu}_{17.5} \mathrm{Ni}_{10} \mathrm{Al}_{7.5}$ and of Schaaff (2001) on Fe diffusion in the same material. By contrast, in conventional metallic glasses no significant deviations from linear Arrhenius behavior have been detected, since the supercooled liquid state is not accessible due to the onset of crystallization (Sec. IV).

As recognized by Knorr, Macht, and Mehrer (1999) and Knorr et al. (2000), the diffusion times applied at low temperatures were too short to reach the metastable state of the supercooled liquid at these temperatures. This is demonstrated in Fig. 9, which shows the TTT diagram for the glass transition and for crystallization of Vitreloy 4 according to Busch and Johnson (1998). Superimposed on this diagram are the annealing times and temperatures applied in the diffusion studies mentioned above. All diffusion anneals were performed in such a way that crystallization was avoided. In Fig. 9, open symbols mark annealing parameters that led to diffusivities below the transition temperature pertaining to the glassy state. Solid symbols and crosses mark annealing parameters that led to diffusivities above the transition temperature, thus representing $D$ values of the supercooled liquid state. Hence the break in the Arrhenius 


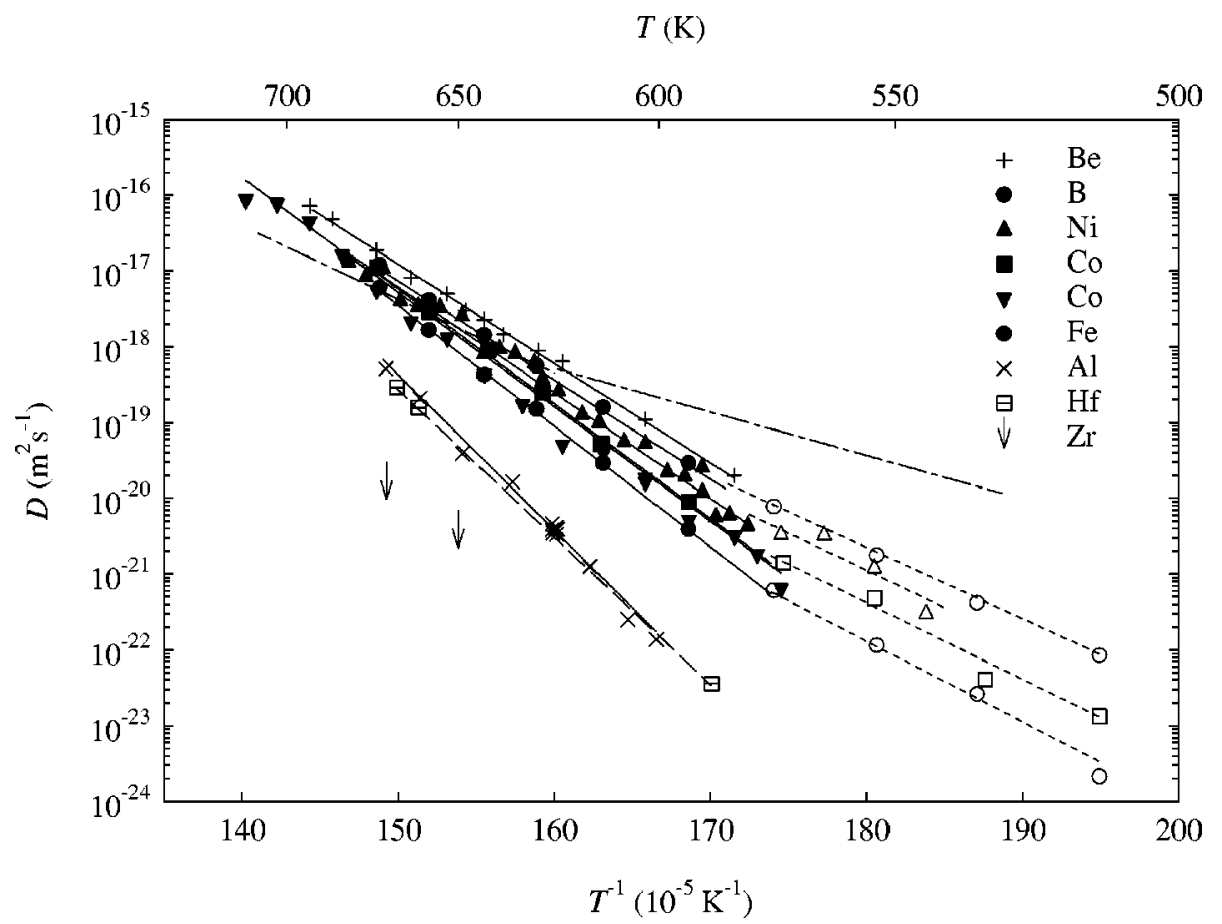

FIG. 8. Arrhenius diagram for tracer diffusion in Vitreloy 4: Be (Rehmet et al., 2001), B (Fielitz et al., 1999), Ni (Knorr, Macht, Freitag, and Mehrer, 1999, 2000), Co (Fielitz et al., 1999), Co (Ehmler et al., 1999), Fe (Fielitz et al., 1999), Al (Budke et al., 1997), Hf (Zumkley, Macht, et al., 2000), Zr (Knorr, Macht, Freitag, and Mehrer, 1999; Knorr, Macht, and Mehrer, 1999, 2000). The dashed-dotted line represents interdiffusion data obtained from Be-Vitreloy 4 diffusion couples (Geyer et al., 1996).

diagram indeed reflects the transition from the glassy to the supercooled liquid state.

A crucial test of this interpretation of the nonlinear Arrhenius behavior of diffusion in bulk metallic glasses was performed by Zumkley et al. (2001). These authors investigated the time dependence of the diffusivities of Fe and B in Vitreloy 4 at low temperatures. For suffi- ciently long annealing times, the material should finally relax into the supercooled liquid state, which then determines the diffusion behavior. Figure 10 shows the temperature dependence of $\mathrm{Fe}$ and B diffusion in Vitreloy 4. Open symbols represent diffusivities measured by Fielitz et al. (1999) in as-cast material. Solid symbols represent diffusivities reported by Zumkley et al. (2001) for Vitre-

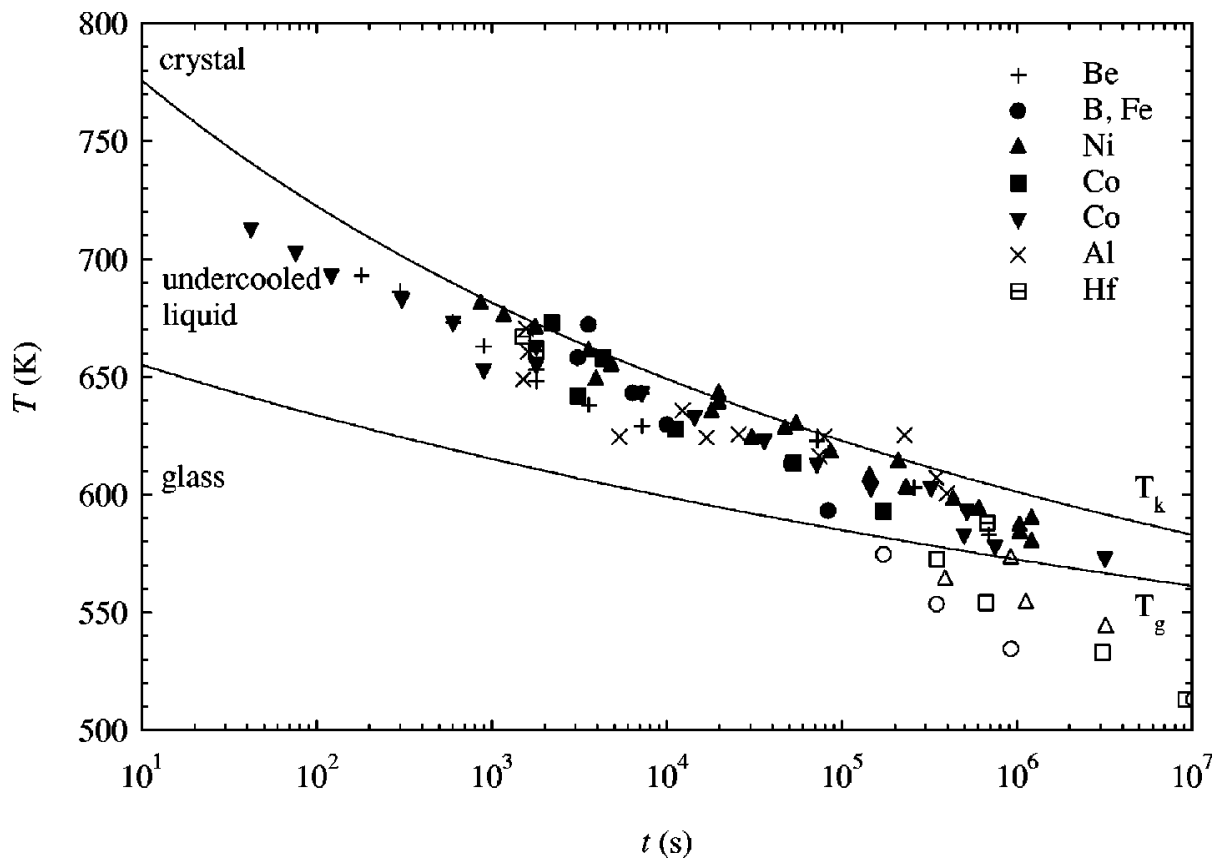

FIG. 9. Time-temperature transformation diagram of Vitreloy 4. The upper line corresponds to the onset of crystallization and separates the undercooled liquid from the crystallized state. The lower line corresponds to the glass transition (Busch and Johnson 1998). Diffusion parameters of various tracer diffusion studies are indicated by solid and open symbols (see text) and by crosses. Be (Rehmet et al., 2001), B, Fe (Fielitz et al., 1999), Ni (Knorr, Macht, Freitag, and Mehrer, 1999; Knorr, Macht, and Mehrer, 1999, 2000), Co (Fielitz et al., 1999), Co (Ehmler et al., 1999), Al (Budke et al., 1997), Hf (Zumkley, Naundorf, and Macht, 2000). 


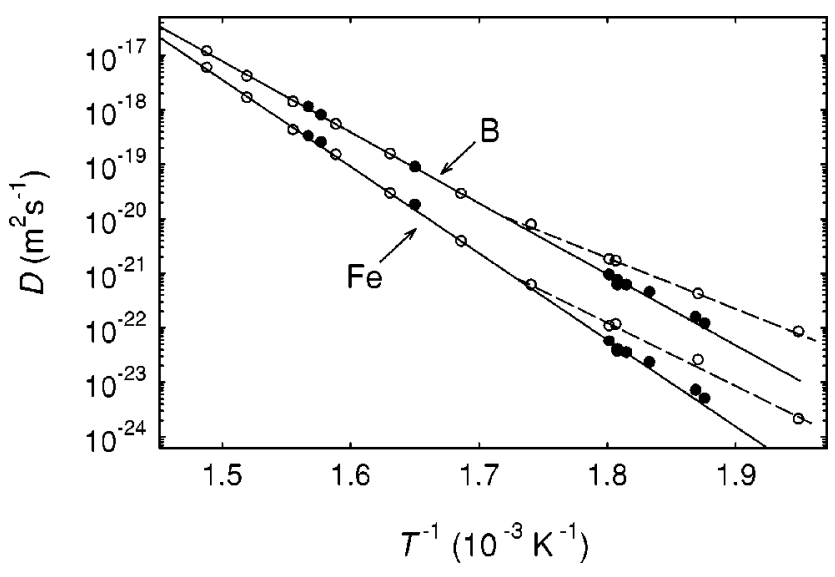

FIG. 10. Arrhenius diagram for diffusion of $\mathrm{B}$ and Fe in Vitreloy 4. Solid symbols pertain to relaxed materials (Zumkley, et al., 2001), open symbols to as-prepared material (Fielitz et al., 1999).

loy 4 which underwent preannealing treatments between $1.17 \times 10^{6} \mathrm{~s}$ and $2.37 \times 10^{7} \mathrm{~s}$ at $553 \mathrm{~K}$, i.e., below the caloric glass temperature. The diffusion coefficients obtained for this super-relaxed material at and below 553 $\mathrm{K}$ are smaller by about $50-80 \%$ than those of the ascast material. In the high-temperature range, the diffusivities in the as-cast and in the relaxed materials coincide. Furthermore, the diffusivities of $\mathrm{Fe}$ and $\mathrm{B}$ in the relaxed material can be described by one Arrhenius equation, which also fits the high-temperature data of the as-cast material. An equivalent explanation of the results in Fig. 9 is that the fictive temperature in a wellannealed glass is low. If it had been possible to measure the diffusivity at still lower temperatures, a kink might have been observed.

It is important to note that the linear Arrhenius behavior observed within experimental accuracy in the supercooled liquid state is due to the limited temperature range of the experiments. Molecular-dynamics simulations (Sec. IX) and mode-coupling theory (Götze and Sjölander, 1992) clearly show a downward curvature at higher temperatures. If an activation enthalpy is attributed to diffusion in the supercooled liquid state, it should be considered as an effective one defined by $-k_{B}$ times the slope of the Arrhenius diagram. It is strongly increased by structural changes occurring above $T_{g}$. The slope could only be considered a "true" activation enthalpy if the structure did not change with temperature.

\section{TEMPERATURE DEPENDENCE OF DIFFUSION}

\section{A. Introduction}

A remarkable feature of experimentally obtained diffusion coefficients $D$ in relaxed melt-spun metallic glasses (also called conventional metallic glasses) is their Arrhenius-type temperature dependence (Sec. III) in the temperature ranges investigated:

$$
D=D_{0} \exp \left(-H / k_{B} T\right),
$$

where $D_{0}, H, k_{B}$, and $T$ are the preexponential factor, the activation enthalpy for diffusion, Boltzmann's constant, and the temperature of diffusion, respectively. ${ }^{2}$ The seeming non-Arrhenius temperature dependence of diffusion observed in some of the early investigations, e.g., for $\mathrm{B}$ diffusion in $\mathrm{Fe}_{40} \mathrm{Ni}_{40} \mathrm{~B}_{20}$ (Cahn et al., 1980) and $\mathrm{Ni}_{59.5} \mathrm{Nb}_{40.5}$ (Kijek et al., 1986), was attributed to an unnoticed time dependence of diffusivity in insufficiently relaxed states of the as-quenched amorphous alloy specimens used for these diffusion measurements (Horváth and Mehrer, 1986; Sharma et al. 1992; see also Sec. III). Further, these measurements were carried out below the glass transition temperature $\left(T_{g}\right)$ due to the limitations imposed by the incipient crystallization of the glass at higher temperatures. On the other hand, in novel bulk metallic glasses (Zhang et al., 1991; Peker and Johnson, 1993) diffusion measurements were carried out over a temperature range extending also into the supercooled liquid region above the glass transition temperature, and as discussed in Sec. III, a kink in the $\ln D$ vs $T^{-1}$ curve was observed at $T_{g}$. The nonlinear Arrhenius region can, however, be split into two linear Arrhenius regions lying below and above the "kink temperature," and these regions represent the behavior in the metastable glassy and supercooled liquid states, respectively (Geyer et al., 1995; Nakajima et al., 1997; Ehmler et al., 1998; Fielitz et al., 1999; Knorr, Macht, and Mehrer, 1999, 2000). The Arrhenius-type temperature dependence below and above $T_{g}$ is not necessarily expected in view of the distribution of barrier heights in amorphous structures (Kronmüller and Frank, 1989) and the structural changes taking place above the glass transition temperature (Geyer et al., 1996). In fact, molecular-dynamics simulation studies of diffusion in metallic glasses have also suggested a curvature at higher temperatures (Teichler, 1997; see also Sec. IX). As regards the nonlinear Arrhenius behavior in bulk metallic glasses, in a recent study the origin of the kink in the Arrhenius plots of diffusivities has been traced to insufficient relaxation of the glassy phase by demonstrating that the kink disappeared as a result of "long-time relaxation" (Zumkley et al., 2001; see also Sec. III).

This section examines the temperature dependence of diffusion coefficients in both the glassy state (mostly in conventional metallic glasses) and the supercooled liquid state (in bulk amorphous alloys). Diffusion of hydrogen, which is an interstitial diffuser, is not considered here; details on hydrogen diffusion in amorphous alloys may be found elsewhere (Berry and Pritchet, 1981; Kirchheim et al., 1982). A discussion of the preexponential factor $D_{0}$ and the activation enthalpy $H$, and on their inter-relationship, which contrasts sharply with that found for crystalline alloys, is also presented in this section. For the temperature dependence of diffu-

\footnotetext{
${ }^{2}$ See, for example, Horváth, Pfahler, et al., 1985; Pfahler et al., 1985; Horváth and Mehrer, 1986; Mehrer and Doerner, 1989; Horváth, 1990; Cahn, 1991; Tyagi et al., 1991a; Faupel, 1992; Sharma et al., 1992, 1994; Mehrer, 1996; Frank, 1997.
} 


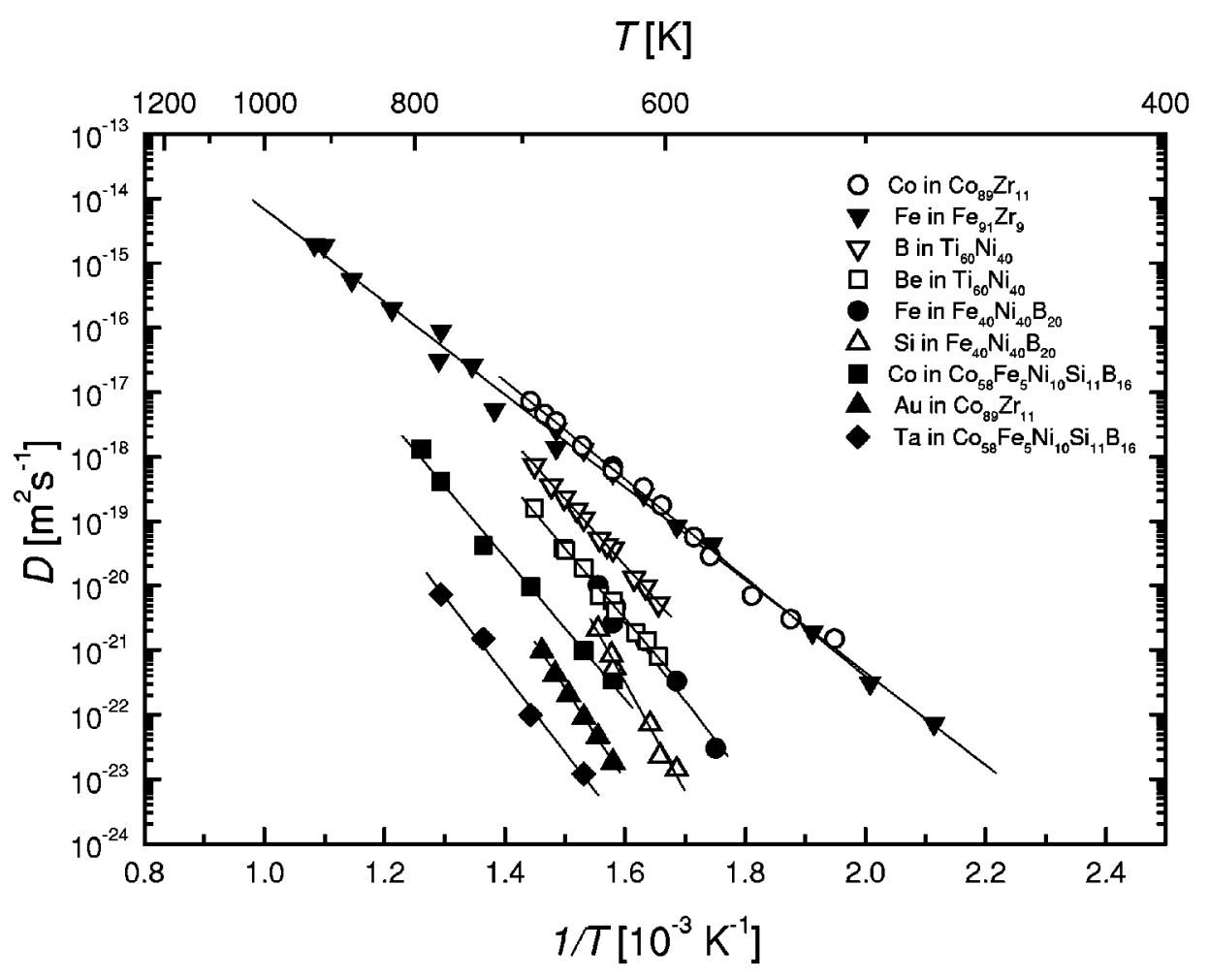

FIG. 11. Some typical plots showing the Arrhenius-type temperature dependence of self-diffusion and impurity diffusion in several metalmetalloid- and metal-metaltype relaxed amorphous alloys: [0] Fe (Horváth and Mehrer, 1986) and $[\triangle]$ Si (Sharma et al., 1992) in $\mathrm{Fe}_{40} \mathrm{Ni}_{40} \mathrm{~B}_{20} ; \quad[\mathbf{\square}]$ $\mathrm{Co}$ and $[\bullet]$ Ta in

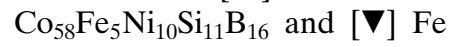
in $\mathrm{Fe}_{91} \mathrm{Zr}_{9}$ (Scharwaechter et al., 1996a); [O] Co and [A] $\mathrm{Au}$ in $\mathrm{Co}_{89} \mathrm{Zr}_{11}$ (Dörner and Mehrer, 1991); [V] B and [ $\square]$ Be in $\mathrm{Ti}_{60} \mathrm{Ni}_{40}$ (Sharma et al., 1994). sion during structural relaxation, irradiation, pressure, and isotope-effect measurements, the reader should see the respective sections in this review.

\section{B. Diffusion in the glassy state}

Diffusion coefficients in the structurally relaxed glassy state of conventional metallic glasses follow an Arrhenius temperature dependence [Eq. (4.1)] thus yielding the diffusion parameters $D_{0}$ and $H$ (Horváth, Pfahler, et al., 1985; Horváth, Freitag, and Mehrer, 1985; Pfahler et al., 1985; Horváth and Mehrer, 1986; Mehrer and Dörner, 1989; Cahn, 1991; Faupel, 1992; Limoge, 1992b; Sharma et al., 1992, 1994; Mehrer, 1996; Frank, 1997; Kronmüller, 1998). Some typical examples of experimentally obtained Arrhenius plots for both metal-metal (M-M) -type and metal-metalloid (M-Me) -type amorphous alloys are shown in Fig. 11. Some investigators have also presented normalized Arrhenius plots (temperature $T$ normalized to the glass transition temperature $T_{g}$ or crystallization temperature $T_{x}$ ), and the activation enthalpy $H$ was directly related to the thermal stability of the glass (Akhtar et al., 1982; Cantor and Cahn, 1983; Sharma et al., 1992, 1994). The temperature range in which diffusion measurements are performed is usually limited to less than $200 \mathrm{~K}$, because at high temperatures the onset of crystallization and at low temperatures the very small atomic mobility prevents meaningful measurements. Consequently, the error margins imposed on the diffusion parameters are relatively large, of the order of $0.2 \mathrm{eV}$ for the activation enthalpy $H$ and of about one order of magnitude for the preexponential factor $D_{0}$ (Sharma et al., 1992; Zumkley et al., 2001). It was shown that the height distribution of barriers to be surmounted in individual diffusion jumps in the disordered structure of the amorphous alloys which is compatible with these experimental error bars, should be fairly narrow, leading to the observed Arrhenius-type temperature dependence of diffusion (Kronmüller and Frank, 1989; van den Beukel, 1994; Frank, 1997). Other reasons suggested for the linearity of the Arrhenius plots include (i) compensation effects between site and saddle-point disorder (Limoge and Bocquet, 1990), and (ii) cooperative atomtransport mechanisms leading to averaging effects in the atomic migration processes (Frank, 1997).

In general, reported values of the activation enthalpy $H$ in various amorphous alloys range from 1 to $3 \mathrm{eV}$ for different diffusants (excluding hydrogen) and seem to follow the size rule for diffusing atoms (Sec. V). The preexponential factor $D_{0}$ shows a wide variation from about $10^{-15} \mathrm{~m}^{2} \mathrm{~s}^{-1}$ to $10^{+13} \mathrm{~m}^{2} \mathrm{~s}^{-1}$ (Sharma et al., 1989, 1993, 1994; Horváth, 1990; Sharma and Faupel, 1999). This large variation in values of $D_{0}$ may indicate different structures and even different diffusion mechanisms (Fedorov, 1970; Hood, 1978; Kronmüller and Frank, 1989; Naundorf et al., 1998, 1999). Further discussion on this point is presented in Sec. IV.D.

One of the popular approaches to explaining atomic mobility in amorphous systems is the free-volume model of Cohen and Turnbull, which was originally applied to liquids (Cohen and Turnbull, 1959; Cohen and Grest, 1981) and later was extended to solid amorphous alloys (Spaepen, 1981, 1988; Stelter and Lazarus, 1987; Duine et al., 1992, 1993; van den Beukel, 1994). According to this approach, both diffusivity and viscosity in amorphous alloys are mediated by similar kinds of defects, namely, the so-called diffusion defects and flow defects, 


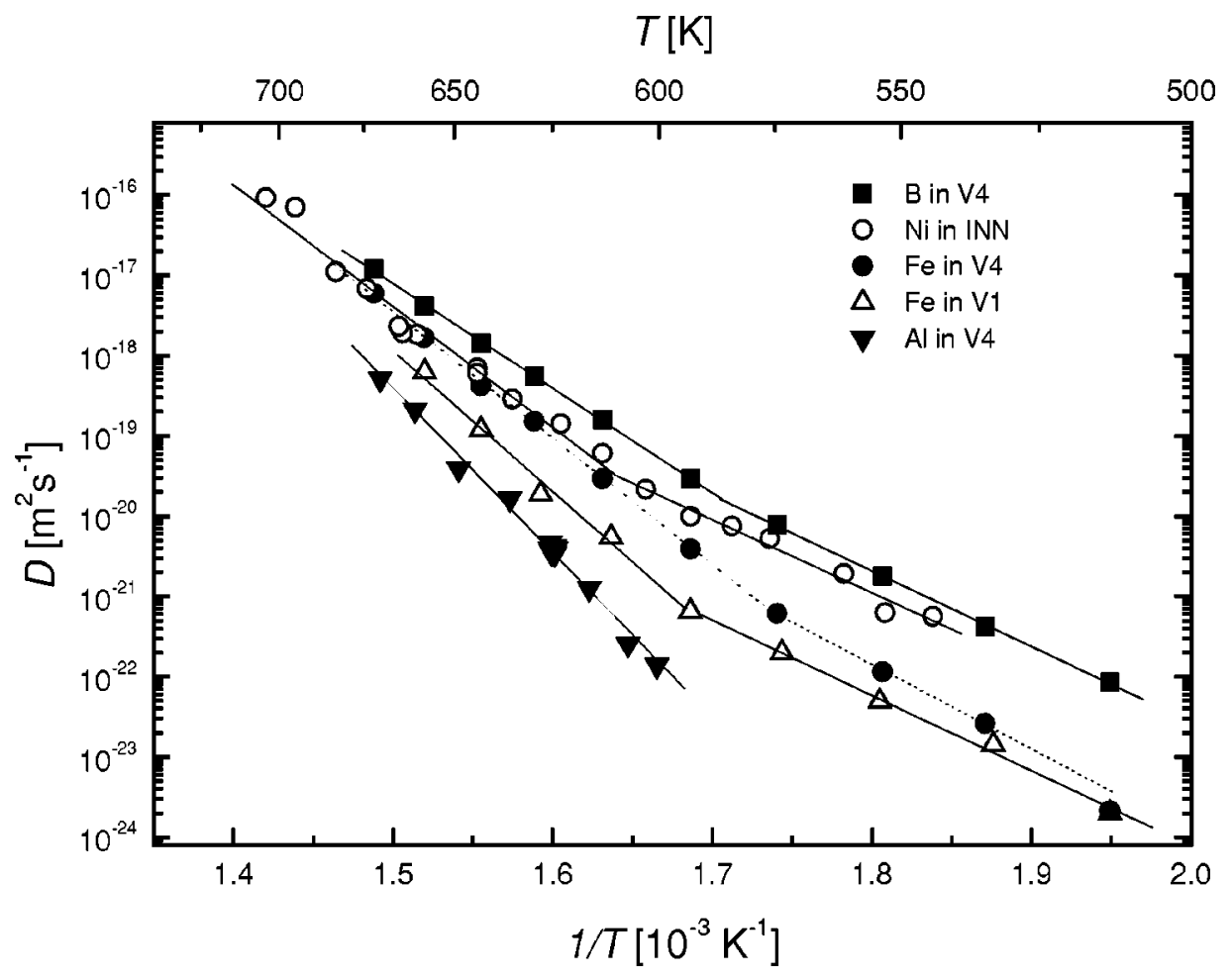

FIG. 12. Some typical plots showing the temperature dependence of self-diffusion and impurity diffusion in several bulk metallic glasses:

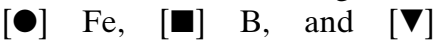
$\mathrm{Al}$ in Vitreloy 4 (V4), i.e., $\quad \mathrm{Zr}_{46.75} \mathrm{Ti}_{8.25} \mathrm{Cu}_{7.5} \mathrm{Ni}_{10} \mathrm{Be}_{27.5}$ (Fielitz et al., 1999); [ $\triangle \mathrm{Fe}$ in Vitreloy 1 (V1), i.e., $\mathrm{Zr}_{41} \mathrm{Ti}_{14} \mathrm{Cu}_{12.5} \mathrm{Ni}_{10} \mathrm{Be}_{22.5}$ (Fielitz et al., 1999); [○] $\mathrm{Ni}$ in the Inoue alloy (INN), i.e., $\mathrm{Zr}_{65} \mathrm{Cu}_{17.5} \mathrm{Ni}_{10} \mathrm{Al}_{7.5} \quad$ (Knorr, Macht, Freitag, and Mehrer, 1999). The two separate Arrhenius regions below and above the "kink temperature" are noteworthy.

respectively, which result from free-volume fluctuations and are frozen in upon cooling the alloy through the glass transition (Spaepen, 1981, 1988; van den Beukel, 1994). The viscosity ( $\eta$ ) has been shown to follow a Vogel-Fulcher-Tammann (VFT) -type equation, $\eta$ $\propto \exp \left[B_{0} /\left(T-T_{0}\right)\right]$, where $T_{0}$ and $B_{0}$ are compositiondependent positive constants (van den Beukel, 1994; Busch et al., 1999; Masuhr et al., 1999). The Arrhenius expression for the diffusion coefficient [Eq. (4.1)] was modified according to the free-volume approach to include the diffusion-defect concentration, $c_{D}$ $\propto \exp \left[-B_{0} / 2\left(T-T_{0}\right)\right]$ (Spaepen, 1981, 1988; van den Beukel, 1991, 1994; Duine et al., 1993), leading to

$$
D=D_{0}^{\prime} c_{D} \exp \left(-H / k_{B} T\right),
$$

where $D_{0}^{\prime}$ represents a preexponential factor not to be confused with the preexponential factor $D_{0}$ in Eq. (4.1) (Sec. IV.D). It was further shown that the StokesEinstein relation between diffusion coefficient and viscosity, $D \eta / k_{B} T=$ const, does not hold in conventional amorphous alloys, irrespective of whether they are relaxed or not (Duine et al., 1995). The failure of the Stokes-Einstein relation clearly points to the fact that hydrodynamic flow does not govern the atomictransport mechanisms in conventional amorphous alloys. Though the free-volume approach broadly quantifies the concentration of the so-called diffusion and flow defects, in the absence of a proper theoretical framework an unambiguous definition of defects in amorphous alloys, similar to that of point defects in crystals, is still elusive.

\section{Diffusion in the supercooled liquid state}

In bulk metallic glasses, it is possible to carry out diffusion measurements in a temperature range well above the glass transition temperature, i.e., in the supercooled liquid region (see, for example, Geyer et al., 1995; Budke et al., 1997; Nonaka et al., 1997; Ehmler et al., 1998; Fielitz et al., 1999; Knorr, Macht, et al., 1999; Knorr et al., 2000; Zumkley, Naundorf, and Macht, 2000). Some typical plots showing the temperature dependence of the diffusion coefficient in several bulk amorphous alloys, namely, $\mathrm{Zr}_{46.75} \mathrm{Ti}_{8.25} \mathrm{Cu}_{7.5} \mathrm{Ni}_{10} \mathrm{Be}_{27.5}$, $\mathrm{Zr}_{41} \mathrm{Ti}_{14} \mathrm{Cu}_{12.5} \mathrm{Ni}_{10} \mathrm{Be}_{22.5}$, and $\mathrm{Zr}_{65} \mathrm{Cu}_{17.5} \mathrm{Ni}_{10} \mathrm{Al}_{7.5}$ (also known as Vitreloy 4, Vitreloy 1, and Inoue alloy, respectively), are displayed in Fig. 12 (also see Fig. 8 in Sec. III showing the temperature dependence for several diffusants in the bulk amorphous alloy Vitreloy 4). The temperature dependence of the diffusivities in these alloys can be split into two different linear Arrhenius regions below and above a "kink temperature," which correspond to the glassy and the supercooled liquid states, respectively (Geyer et al., 1996; Fielitz et al., 1999; Knorr, Macht, et al., 1999; Knorr et al., 2000; Zumkley, Naundorf, and Macht, 2000; Sec. III). As in conventional amorphous alloys (Sec. IV.B) the preexponential factor $D_{0}$, obtained from the Arrhenius fits for both amorphous and supercooled liquid states of several bulk glasses, show a wide variation from about $10^{-15} \mathrm{~m}^{2} \mathrm{~s}^{-1}$ to $10^{+15} \mathrm{~m}^{2} \mathrm{~s}^{-1}$, while the activation enthalpies $H$ vary between 0.6 and $4.5 \mathrm{eV}$ (Fielitz et al., 1999; Sharma and Faupel, 1999; Zumkley, Naundorf, and Macht, 2000), obeying a size rule for the diffusing atoms (Sec. V). The activation enthalpy and preexponential factor in the supercooled liquid state are higher than those in the amorphous state, and the diffusivities in the amorphous state are higher than those expected from a linear lowtemperature extrapolation of the Arrhenius behavior from the deeply supercooled liquid region to lower temperatures (see Fig. 12 and also Fig. 8 in Sec. III.). The 
kink temperature separating the amorphous and deeply supercooled liquid regions in bulk metallic glasses is higher for species diffusing faster in the amorphous phase (Fielitz et al., 1999; Geyer et al., 1999). This appears to reflect the dependence of the caloric glass transition temperature on the time scales involved for diffusion and relaxation processes (Masuhr et al., 1999).

It is noteworthy that the Stokes-Einstein relation between diffusivity and viscosity was not found to hold for Be diffusion in the supercooled liquid state of the bulk amorphous alloy Vitreloy 4 and the related alloy Vitreloy 1 (Geyer et al., 1996). This result supports the view of a decoupling of diffusion and viscous flow in the supercooled liquid state (Busch et al., 1999; Masuhr et al. 1999). In some of the early work on diffusion in bulk metallic glasses, the kink in the Arrhenius plots near the glass transition temperature was interpreted in terms of the temperature dependence of the time scales for viscous flow and for the diffusion of medium-sized atoms, and a change in the diffusion mechanism at the glass transition temperature was suggested (Geyer et al., 1999; Masuhr et al., 1999). Below the kink temperature, the matrix was assumed to be rigid on the scale of the time elapsing between two consecutive successful diffusional jumps (i.e., the characteristic diffusion time $\tau_{D}$ was much smaller than the matrix relaxation time $\tau_{\eta}$ as determined from viscosity measurements), and diffusion was envisaged to proceed by thermally activated singleatom hopping. Furthermore, it was proposed that above the kink temperature additional transport by cooperative atom movements starts dominating (Busch et al., 1999; Geyer et al., 1999; Greer, 1999; Masuhr et al., 1999; Tang et al., 1999). Tang et al. (1999) and Greer (1999) attributed the proposed change in the diffusion mechanism at $T_{g}$ to the effect of the glass transition on the diffusion behavior.

As discussed in Sec. III.B.2, there is clear evidence now that the kink in the temperature dependence of diffusivity data is not related to such a change in the diffusion mechanism, but depends on the thermal history of the material and is caused by incomplete relaxation to the (metastable) equilibrium state of the supercooled liquid.

\section{Correlation between $D_{0}$ and $H$}

The observed values of the preexponential factor $D_{0}$ in amorphous alloys show a much larger variation (from about $10^{-15}$ to $10^{+15} \mathrm{~m}^{2} \mathrm{~s}^{-1}$ ) than those reported for crystalline alloys (about $10^{-6}-10^{+2} \mathrm{~m}^{2} \mathrm{~s}^{-1}$ ), and thus values of $D_{0}$ in amorphous and crystalline systems belonging to the same value of $H$ may differ by several orders of magnitudes (Borg and Dienes, 1988; Sharma et al., 1989, 1993, 1994; Horváth, 1990; Sharma and Faupel, 1999). The experimental values of $D_{0}$ and $H$ in amorphous alloys have been found to follow the relationship (Sharma et al., 1989; Naundorf et al., 1998, 1999; Sharma and Faupel, 1999)

$$
D_{0}=A \exp (H / B) \text {, }
$$

where $A$ and $B$ are constants. This relationship has a universal character in the sense that it has been found to be valid not only in amorphous alloys (Sharma et al., 1992, 1994; Naundorf et al., 1998, 1999) but also for selfand impurity diffusion in crystalline alloys involving both substitutional and interstitial solid solutions (Fedorov, 1970; Dariel, 1974; Hood, 1978). The values of $D_{0}$ and $H$ both in conventional amorphous alloys and in the glassy and the supercooled liquid states of bulk amorphous alloys do follow the same relationship, as shown in Fig. 13 (Sharma and Faupel, 1999). Interestingly, values of the fitting parameters $A\left(10^{-7} \mathrm{~m}^{2} \mathrm{~s}^{-1}\right.$ in crystalline alloys as compared to $10^{-19}-10^{-20} \mathrm{~m}^{2} \mathrm{~s}^{-1}$ in amorphous alloys $)$ and $B(0.41 \mathrm{eV}$ in crystalline alloys as compared to $0.055 \mathrm{eV}$ in amorphous alloys) are quite different for amorphous and corresponding crystalline systems, and are unique and distinct for each system (Fedorov, 1970; Hood, 1978; Sharma et al., 1989, 1993; Sharma and Faupel, 1999). It should be noted that the fitting parameter $A$ may have an error margin of about one order of magnitude, while the parameter $B$ may only deviate by about $10 \%$, primarily due to the errors in $D_{0}$ and $H$ (Sharma et al., 1994).

For diffusion via vacancies or interstitials in crystals, the constants $A$ and $B$ have been accounted for in terms of Zener's theory of the pre-exponential factor (Shewmon, 1989). In this approach the preexponential factor $D_{0}^{\prime}$ (which for crystals is identical with $D_{0}$ ) is expressed as

$$
D_{0}=g a^{2} f \nu_{0} \exp \left(\Delta S / k_{B}\right),
$$

where $g$ is a geometric factor, $a$ the effective jump distance, $\nu_{0}$ the effective jump attempt frequency, $f$ the correlation factor, and $\Delta S$ the entropy of diffusion. For crystals, $\Delta S=-\beta_{\mu} H$ was derived, where $\beta_{\mu}$ represents the temperature dependence of the shear modulus (Zener, 1951; Fedorov, 1970; Hood, 1978; Naundorf et al., 1998, 1999). This interpretation yields

$$
A=g a^{2} f \nu_{0} \text { and } B=-k_{B} / \beta_{\mu},
$$

and fits well to the experimental data for diffusion in crystals (Naundorf et al., 1998, 1999).

Basically, Eq. (4.5) should hold also for amorphous alloys, since its derivation does not explicitly refer to the crystalline structure or specific jump mechanisms, and the elastic behavior, which essentially determines the relation, is similar when the temperature dependence of the shear modulus of crystals and amorphous alloys is compared (Chen, 1978). However, the fact that the values of the constants $A$ and $B$ differ considerably for amorphous alloys and crystalline systems indicates that the diffusion mechanism in amorphous alloys must be different from the vacancy or interstitial mechanism operating in crystals.

An attempt was made to interpret the values of the fitting parameters $A$ and $B$ [Eq. (4.3)] in terms of the free-volume approach. According to this approach the value of the parameter $A$ can be expressed as $A$ $=g a^{2} f \nu_{0} c_{d}$ using Eqs. (4.1)-(4.4) (van den Beukel, 1994; Sharma and Faupel, 1999), and it was indeed pos- 


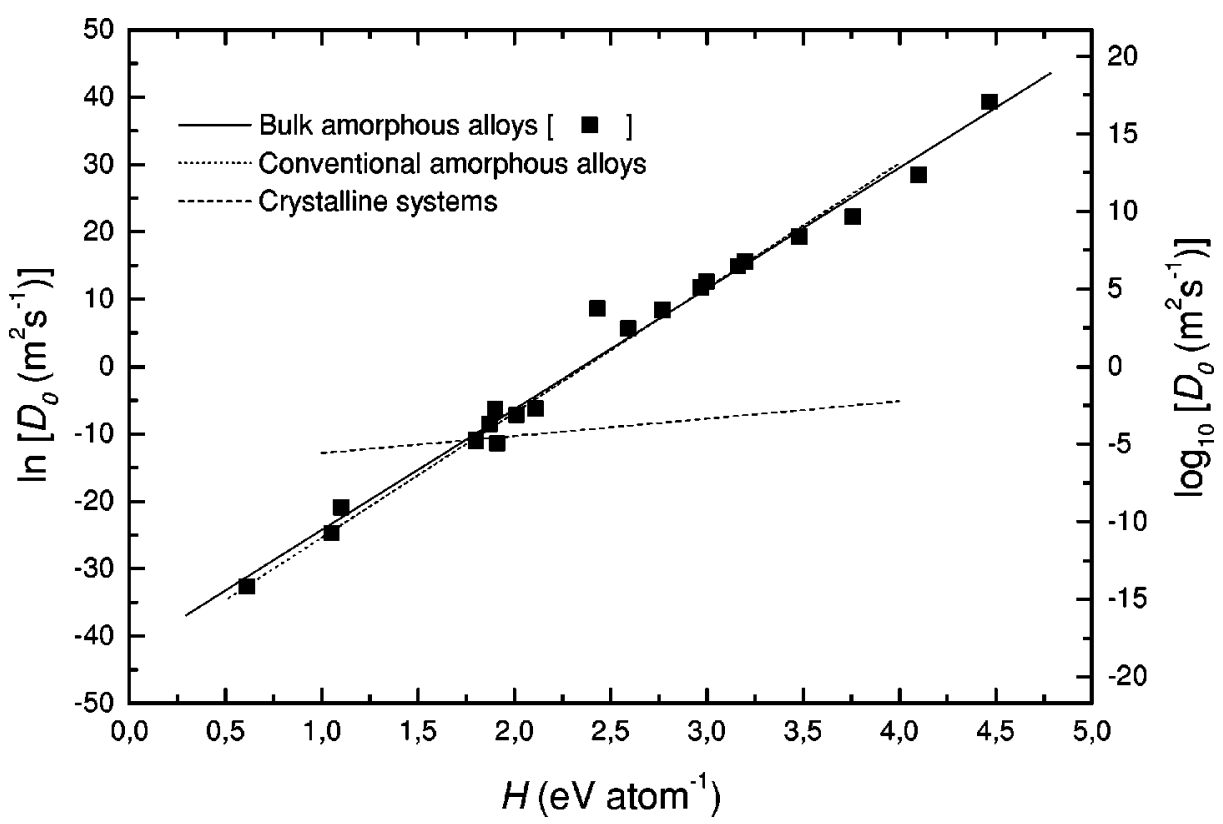

FIG. 13. Typical plots showing the correlation between $\ln D_{0}$ and $H$ for amorphous and crystalline alloys (Sharma and Faupel, 1999): dotted line, conventional amorphous alloys (Sharma et al., 1989, 1993, 1994); solid line, bulk amorphous alloys (Sharma and Faupel, 1999); dashed line, crystalline systems (Sharma et al., 1993, 1994). The solid line represents a linear fit to the data points 口 in bulk metallic glasses, while the dotted and dashed lines represent linear fits to data points (not shown here) in conventional amorphous alloys and crystalline systems, respectively. The noteworthy feature is the distinct nature of the $D_{0}-H$ correlation for amorphous and crystalline systems.

sible to reconcile the observed low value of $A$ $\sim 10^{-19}-10^{-20} \mathrm{~m}^{2} \mathrm{~s}^{-1}$ in glassy amorphous alloys to within an order of magnitude by assuming a temperature-independent value of $c_{D}$ (Sharma and Faupel, 1999). In these terms the defect concentration $c_{D}$ plays a major role in explaining the very low value of $A$, but at the moment there is no independent and direct evidence for the presence of such defects in amorphous alloys.

As indicated by Eqs. (4.3) and (4.4), the constant $B$ can be related to the entropy of diffusion $\Delta S$, viz., $B$ $=k_{B} H / \Delta S$. The value of $B$ obtained for supercooled liquids and amorphous alloys suggests a rather high value of $\Delta S$ ranging from $19 k_{B}$ to $56 k_{B}$ for typical $H$ values $(1-4 \mathrm{eV})$, unlike much lower values of $2.5-7.5 k_{B}$ for crystalline alloys (Frank et al., 1988; Sharma et al., 1989, 1993; Sharma and Faupel, 1999). These higher values of $\Delta S$ in glassy systems are indicative of the extended nature of the defects in these materials (Seeger and Chik, 1968) and point to a collective diffusion mechanism involving many atoms (Frank et al., 1988; Sharma et al., 1989, 1993; Naundorf et al., 1998, 1999; Sharma and Faupel, 1999).

Therefore, irrespective of a firm physical basis for the interpretation of the values of the parameters $A$ and $B$ [Eq. (4.3)], the distinct nature of the relationship between $\ln D_{0}$ and $H$ in amorphous and crystalline alloys clearly points to different diffusion mechanisms in the two cases and certainly does not support a mechanism based solely on single-atom jumps in relaxed amorphous alloys.

\section{SIZE DEPENDENCE}

\section{A. Remarks on atomic size}

This section is devoted to the observation that the diffusivities of solutes depend on their sizes: In the same amorphous alloy, "smaller" atoms tend to diffuse faster than "bigger" ones (Sharma et al., 1994). What does "smaller" or "bigger" size mean? The size of a single atom is, in principle, an undetermined quantity due to the diffuse electron distribution around it. Nevertheless an average size of atoms can be defined and measured for a solid using certain macroscopic properties of many atoms, e.g., the mass density or x-ray diffraction pattern of crystals. From these, average atomic volumes and atomic diameters, respectively, are derived for the elements (Pearson, 1958; Barrett and Massalski, 1966). The exact average size of an atom, i.e., its diameter or volume, can be different if the solid exists in different modifications, for instance, for Co or Fe. Moreover, the same solute atom has a different size when dissolved in different solvents, pure elements or alloys, due to chemical, i.e., electronic interaction, as is indicated, for example, by a violation of Vegard's rule (Barrett and Massalski, 1966). These observations indicate that the size of an atom is a somewhat ambiguous parameter.

It has been shown that average sizes are correlated with the valence, i.e., with the chemical behavior of the elements according to their position in the Periodic Table. For example, the IB noble metals $\mathrm{Cu}, \mathrm{Ag}$, and $\mathrm{Au}$ are smaller than their right-hand IIB neighbors $\mathrm{Zn}, \mathrm{Cd}$, and $\mathrm{Hg}$, respectively (Barrett and Massalski, 1966). Fig- 


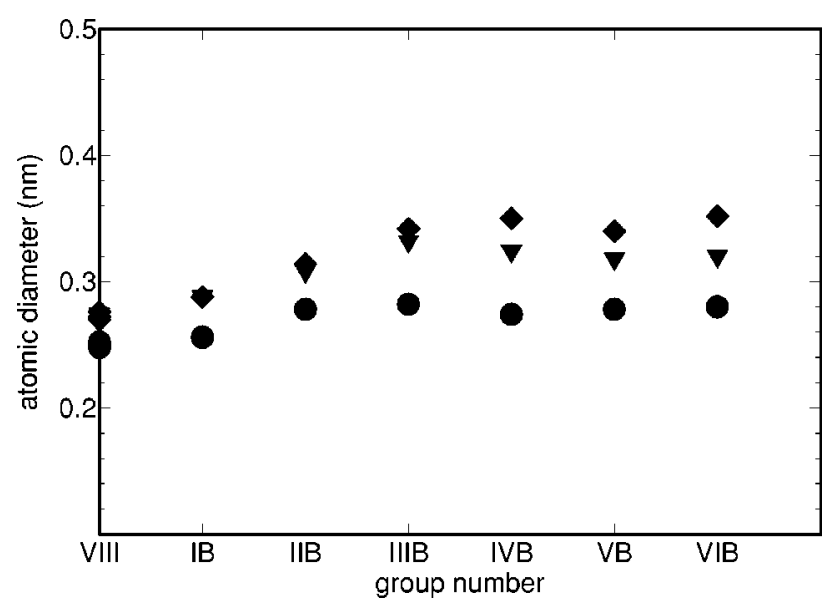

FIG. 14. Size of elements from groups VIII-VIB of the Periodic Table. Periods 4-6 are indicated by solid circles, triangles, and diamonds, respectively. Atomic diameters are taken from Barrett and Massalski, 1966.

ure 14 shows the generally increasing trend of atomic diameters for elements with group numbers VIII-VIB. In this plot, considerable scatter is obvious, which is caused by the additional dependence of size on the period in the Periodic Table. In fact, for chemically similar elements (same group number) in groups IVB-VIB, the size increase with increasing period number is larger than the general increase with group number in the same period. In spite of all the ambiguities the atomic diameter of the elements, as given, for example, by Pearson (1958) and Barrett and Massalski (1966), offers a convenient scheme-which is also adopted here-for ranking properties that are expected to vary systematically across the Periodic Table.

In this section we focus on the dependence of the activation enthalpy $H$ on the size of solutes. With regard to the empirical relation which exists between the two parameters of the diffusion coefficient, the activation enthalpy $H$ and the preexponential factor $D_{0}$ (Sharma et al., 1989; Naundorf et al., 1998, 1999; Sec. IV.D), we believe that the size dependence of the activation enthalpy reflects essential features of the actual diffusion mechanism. The influence of the size of the diffusant on diffusion in crystals (Lazarus, 1960; Le Claire, 1962; Warburton and Turnbull, 1975) is also briefly described. By comparison, these results may serve as a guideline which gives valuable suggestions for possible diffusion mechanisms in amorphous alloys.

\section{B. Solutes in crystalline metals}

The diffusion mechanism of solutes in crystals is basically determined by the size of the solute in two ways: directly by the elastic strains the solute exerts on the surrounding matrix due to its size misfit, and indirectly by the electronic interaction between the solute and a diffusion-mediating vacancy due to the different valences (excess charge) of solvent and solute (Lazarus, 1960; Le Claire, 1962; Shewmon, 1963), which can be

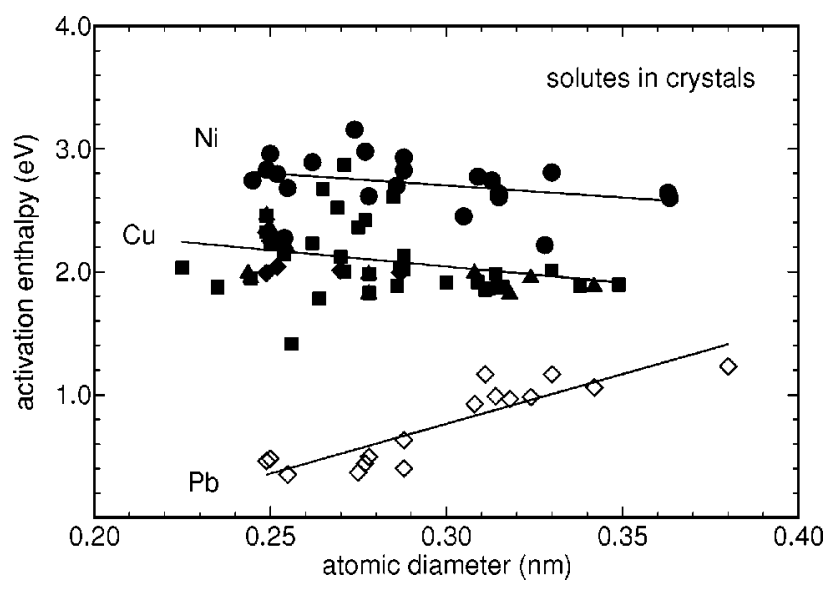

FIG. 15. Size dependence of the activation enthalpy of solute diffusion in crystalline metals. Atomic diameters of solutes are from Barrett and Massalski (1966), activation enthalpies of solutes from Le Claire and Neumann (1990) (solid circles, in Ni; solid squares, in $\mathrm{Cu}$; open diamonds, in $\mathrm{Pb}$ ), from Almazouzi et al. (1996, 1998) (solid diamonds, in $\mathrm{Cu}$ ), and from Hood (1978) (solid triangles, in $\mathrm{Cu}$ ). Lines are linear least-meansquares fits to the data.

translated into a corresponding size dependence (Fig. 15). From the viewpoint of elastic interaction, two opposing effects are expected. On the one hand, the bigger the diffusing atom the greater the migration enthalpy should be due to the increased elastic work necessary to push the atom through a gate of matrix atoms. On the other hand, the vacancy formation enthalpy is decreased in the compressive strain field of a solute atom bigger than the matrix atoms, which leads in this case to a binding enthalpy between the solute and the vacancy and therefore to a decrease in the enthalpy of diffusion. Since the latter effect can be regarded as small, an increase in the enthalpy of diffusion and a corresponding decrease in the diffusion coefficient with increasing size of the solute is expected. The migration enthalpy $H^{M}$ when the elastic interaction dominates can be conveniently approximated by $H^{M}=\mu \Omega \delta^{2} \quad$ (Zener, 1950; Shewmon, 1963), where $\mu$ is an appropriate elastic modulus and $\Omega$ is the atomic volume of the solvent, while $\delta$ varies with the size of the solute and is the representative strain for the matrix when the solute is in the saddle point.

When the electronic interaction between solute and solvent is dominant, an enhanced diffusivity is expected due to both an increased concentration of vacancies and a lowering of the migration enthalpy when the valence of the solute is greater than that of the solvent, i.e., when the solute is on the right of the solvent in the Periodic Table, and vice versa (Lazarus, 1960; Le Claire, 1962; Neumann and Tölle, 1995). In its basic form, the difference $\Delta H$ between the activation enthalpies of solute and self-diffusion with a valence difference $\Delta Z$ was given as $\Delta H=-\Delta Z \Phi$ (Lazarus, 1960; Le Claire, 1962), where $\Phi$ describes the spatial screening of the surplus valence of the solute and is determined by properties of the solvent. This electronic interaction translates into a trend 
of decreasing activation enthalpy with increasing size if, for instance, the diffusion of group-IIB and group-IIIB solutes in the IB noble metals is considered. Since the relation between group number and size is not unique (Fig. 14), a correspondingly strong scatter of the size dependence of the activation enthalpy is expected in this case.

Figure 15 presents experimental data on the size dependence of the activation enthalpy for diffusion of different solutes in $\mathrm{Ni}, \mathrm{Cu}$, and $\mathrm{Pb}$. The relatively strong scatter of the data points is obvious. However, in spite of this scatter a significant trend (as indicated by the leastmean-squares fit to the data points) of a decreasing activation enthalpy with increasing size is visible for diffusion in $\mathrm{Ni}$ and $\mathrm{Cu}$, while the activation enthalpy of solutes in $\mathrm{Pb}$ increases.

The diffusion behavior in $\mathrm{Cu}$ (and in other IB noble metals) was explained by the electrostatic model, i.e., the interaction between the excess charge of the solute and the vacancy (Lazarus, 1960; Le Claire, 1962; Neumann, 1983; Neumann and Tölle, 1995), as mentioned above. The obvious size dependence observed here is an indirect one and refers to the systematic increase in size with increasing valence (group numbers IB to VB) in the Periodic Table, as shown in Fig. 14. A vacancy mechanism is also generally accepted for diffusion of substitutional solutes in Ni (Le Claire, 1978; Neumann, 1983) and in many other crystalline metals. The similarly decreasing size dependence in $\mathrm{Ni}$ and $\mathrm{Cu}$ with average slopes of about $-2.5 \mathrm{eV} / \mathrm{nm}$ (enthalpy decrease per nanometer increase of the diameter) indicates that the effect of the solutes on the activation enthalpy is largely separated from that of the solvent, as suggested by the electrostatic model. Since these sets of data include the activation enthalpy of self-diffusion, they also reflect the empirical melting-point rule (Shewmon, 1989).

It is quite interesting to compare the decreasing trend of the size dependence in $\mathrm{Ni}$ and $\mathrm{Cu}$ with the increasing trend in $\mathrm{Pb}$, which is a polyvalent and "open" element (Pearson, 1958). The solutes for diffusion in $\mathrm{Pb}$ considered in Fig. 15 are mainly group-IIB to group-VB elements, for which a decreasing trend was observed in $\mathrm{Cu}$. Generally these are fast diffusers in $\mathrm{Pb}$, and there is ample evidence, for instance, from the magnitudes of the concentration dependence and isotope effect and from anelastic relaxation, that the diffusion of these solutes is dominated by some interstitial-type mechanism which may include contributions of vacancy-interstitial pairs, or by an interstitialcy-type mechanism, known as a "diplon" mechanism (Warburton and Turnbull, 1975; Le Claire, 1978). The latter mechanism is further supported by the observation that the group-IIB to group-VB diffusants have a smaller atomic diameter than $\mathrm{Pb}$, a condition that was found to be essential for the formation of mixed dumbbells (Dederichs et al., 1978; Schilling, 1978). These are closely related to the diplon and describe an interstitial-type defect consisting of a host atom and a solute that share a lattice site.

The different size dependence of the same diffusants in different solvents, for instance, in $\mathrm{Cu}$ and $\mathrm{Pb}$, is sig- nificant and is determined by different diffusion mechanisms. Obviously in crystals an increasing size dependence can be taken as a sign that the dominant interaction between solute and solvent is elastic, which is typical of an interstitial- or interstitialcy-type diffusion mechanism, while a decreasing size dependence can be taken as a sign that the dominant interaction between solute and solvent is electronic, which is typical of a vacancy mechanism.

\section{Solutes in amorphous alloys}

Table I presents a compilation of activation enthalpies of different solutes in amorphous alloys together with the size of the solutes. Two points are noticeable: The activation enthalpies are of comparable magnitude to those observed in crystals, and in all alloys there is a general trend towards increasing activation enthalpy with increasing size of the solutes. However, considerable deviations from this trend for a single solute are also apparent, for instance, for $\mathrm{Ir}$ and $\mathrm{W}$ in the $\mathrm{Pd}-\mathrm{Cu}-\mathrm{Si}$ glass and for $\mathrm{Au}$ in the Fe-Ni-B glass. These exceptions are of largely unknown origin. They could arise from strong chemical interactions between the particular solute and certain components of the alloy, which conceal the size effect (Sharma et al., 1994). A relatively strong dependence of the solute diffusion on the composition of the alloy appears to be a general feature in amorphous alloys, as is obvious, for instance, in the bulk alloys (Fielitz et al., 1999) and in the Zr-based alloys (Höfler et al., 1992). More data are needed, however, to quantify this observation.

The alloys $\mathrm{Zr}_{61} \mathrm{Ni}_{39}$ and $\mathrm{Ti}_{60} \mathrm{Ni}_{40}$ are closely related. Indeed, as is shown in Fig. 16, the size dependence (as indicated by the linear least-mean-squares fit to the data points) in both alloys is similar. The absolute values of the activation enthalpy, however, are lower by about 1 $\mathrm{eV}$ for the Zr-based alloy than for the Ti-based alloy. This shift reflects the lower melting temperature of the Zr-based alloy (Hansen and Anderko, 1958) and its lower elastic modulus (Hearmon, 1979). It is also in qualitative agreement with the empirical rules known for diffusion coefficients in crystals (Shewmon, 1963), according to which, for the same diffusion mechanism, the activation enthalpy should increase with increasing melting temperature and elastic modulus of the solvent. On the other hand, these rules are violated when the alloys $\mathrm{Zr}_{9} \mathrm{Fe}_{91}$ and $\mathrm{Zr}_{76} \mathrm{Fe}_{24}$ are compared (Table I). Here the activation enthalpies of self-diffusion are generally higher in the $\mathrm{Zr}_{76} \mathrm{Fe}_{24}$ alloy, by about $0.8 \mathrm{eV}$, although the melting temperature as well as the elastic modulus of this alloy are considerably lower than the corresponding quantities of the $\mathrm{Zr}_{9} \mathrm{Fe}_{91}$ alloy (Hansen and Anderko, 1958; Hearmon, 1979).

In Table I data are compiled on solutes from across the Periodic Table in the amorphous metal-metal (M-M) as well as the metalloid-metal (Me-M) alloys. Most of these solutes are smaller than the biggest atoms in the M-M alloys, which are $\mathrm{Zr}$ (0.32 nm diameter) and $\mathrm{Ti}$ (0.292 $\mathrm{nm}$ diameter). In contrast to this, we find that 
TABLE I. Size of solutes and tracers, and their activation enthalpy of diffusion in amorphous alloys. The metallic diameters and the atomic volumes are taken from Barrett and Massalski (1966). The average volumes of the alloys are calculated according to Vegard's rule. The alloy compositions of V1 and V4 are $\mathrm{Zr}_{41} \mathrm{Ti}_{14} \mathrm{Cu}_{12.5} \mathrm{Ni}_{10} \mathrm{Be}_{22.5}$ and $\mathrm{Zr}_{46.8} \mathrm{Ti}_{8.2} \mathrm{Cu}_{7.5} \mathrm{Ni}_{10} \mathrm{Be}_{27.5}$, respectively.

\begin{tabular}{|c|c|c|c|c|c|}
\hline $\begin{array}{l}\text { Alloy } \\
\text { (reference) }\end{array}$ & $\begin{array}{l}\text { Av. volume } \\
10^{-3} \mathrm{~nm}^{3}\end{array}$ & Solute/tracer & $\begin{array}{l}\text { At. volume } \\
10^{-3} \mathrm{~nm}^{3}\end{array}$ & $\begin{array}{l}\text { Metal. diam. } \\
0.1 \mathrm{~nm}\end{array}$ & $\begin{array}{c}\text { Activ. enthalpy } \\
\text { eV }\end{array}$ \\
\hline $\mathrm{Zr}_{76} \mathrm{Fe}_{24}$ & 20.50 & $\mathrm{Fe}$ & 11.78 & 2.52 & 2.32 \\
\hline (Horváth et al., 1988) & & $\mathrm{Zr}$ & 23.27 & 3.20 & 3.22 \\
\hline $\mathrm{Zr}_{61} \mathrm{Ni}_{39}$ & 18.46 & $\mathrm{Cu}$ & 11.81 & 2.56 & 1.33 \\
\hline \multirow[t]{3}{*}{ (Sharma et al., 1989) } & & $\mathrm{Au}$ & 16.97 & 2.88 & 1.66 \\
\hline & & $\mathrm{Al}$ & 16.60 & 2.86 & 1.68 \\
\hline & & $\mathrm{Sb}$ & 30.23 & 3.18 & 2.07 \\
\hline $\mathrm{Zr}_{50} \mathrm{Ni}_{50}$ & 17.11 & $\mathrm{Co}$ & 11.10 & 2.50 & 1.40 \\
\hline \multirow[t]{4}{*}{ (Hahn et al., 1988) } & & $\mathrm{Ni}$ & 10.94 & 2.48 & 1.45 \\
\hline & & $\mathrm{Cu}$ & 11.81 & 2.56 & 1.57 \\
\hline & & $\mathrm{Fe}$ & 11.78 & 2.52 & 1.64 \\
\hline & & $\mathrm{Au}$ & 16.97 & 2.88 & 1.77 \\
\hline $\mathrm{Zr}_{9} \mathrm{Fe}_{91}$ & 12.80 & $\mathrm{Fe}$ & 11.78 & 2.52 & 1.47 \\
\hline (Horváth et al., 1988) & & $\mathrm{Zr}$ & 23.27 & 3.20 & 2.51 \\
\hline V1 & 16.73 & $\mathrm{~B}$ & 7.28 & 1.96 & 2.97 \\
\hline (Geyer et al., 1996; & & $\mathrm{Fe}$ & 11.78 & 2.52 & 3.48 \\
\hline Fielitz et al., 1999) & & $\mathrm{Be}$ & 8.12 & 2.25 & 4.47 \\
\hline V4 & 16.74 & $\mathrm{Be}$ & 8.12 & 2.25 & 1.9 \\
\hline (Geyer et al., 1996; & & $\mathrm{B}$ & 7.28 & 1.96 & 2.59 \\
\hline Fielitz et al., 1999; & & $\mathrm{Ni}$ & 10.94 & 2.48 & 2.77 \\
\hline Knorr et al., 2000; & & $\mathrm{Fe}$ & 11.78 & 2.52 & 3.16 \\
\hline \multirow[t]{3}{*}{ Zumkley, Macht, et al., 2000) } & & $\mathrm{Co}$ & 11.10 & 2.50 & 3.0 \\
\hline & & $\mathrm{Al}$ & 16.60 & 2.86 & 4.1 \\
\hline & & Hf & 22.16 & 3.13 & 3.85 \\
\hline $\mathrm{Ti}_{60} \mathrm{Ni}_{40}$ & 16.34 & $\mathrm{~B}$ & 7.28 & 1.96 & 2.05 \\
\hline \multirow[t]{3}{*}{ (Sharma et al., 1994) } & & $\mathrm{Be}$ & 8.12 & 2.25 & 2.20 \\
\hline & & $\mathrm{Fe}$ & 11.78 & 2.52 & 2.33 \\
\hline & & $\mathrm{Si}$ & 20.04 & 2.64 & 2.35 \\
\hline $\mathrm{Fe}_{40} \mathrm{Ni}_{40} \mathrm{~B}_{20}$ & 10.54 & $\mathrm{Au}$ & 16.97 & 2.88 & 2.09 \\
\hline (Sharma et al., 1992; & & $\mathrm{Ni}$ & 10.94 & 2.48 & 2.14 \\
\hline Akhtar et al., 1982; & & $\mathrm{Be}$ & 8.12 & 2.25 & 2.16 \\
\hline \multirow[t]{5}{*}{ Horváth and Mehrer, 1986) } & & $\mathrm{Fe}$ & 11.78 & 2.52 & 2.35 \\
\hline & & $\mathrm{Cu}$ & 11.81 & 2.56 & 2.73 \\
\hline & & $\mathrm{P}$ & 21.88 & 2.56 & 3.06 \\
\hline & & $\mathrm{Si}$ & 20.04 & 2.64 & 3.39 \\
\hline & & $\mathrm{Ti}$ & 19.94 & 2.92 & 3.82 \\
\hline $\mathrm{Pd}_{78} \mathrm{Cu}_{16} \mathrm{Si}_{6}$ & 14.59 & $\mathrm{Pt}$ & 15.10 & 2.76 & 1.39 \\
\hline \multirow[t]{7}{*}{ (Bøttiger et al., 1988) } & & $\mathrm{Au}$ & 16.97 & 2.88 & 2.10 \\
\hline & & $\mathrm{Tl}$ & 28.59 & 3.42 & 2.70 \\
\hline & & $\mathrm{Hg}$ & 23.39 & 3.14 & 2.79 \\
\hline & & $\mathrm{Pb}$ & 30.33 & 3.50 & 2.98 \\
\hline & & $\mathrm{W}$ & 15.86 & 2.78 & 3.19 \\
\hline & & $\mathrm{Bi}$ & 35.41 & 3.40 & 3.69 \\
\hline & & $\mathrm{Ir}$ & 14.15 & 2.72 & 3.99 \\
\hline
\end{tabular}

in the Me-M alloys, where the biggest atoms are the $\mathrm{Fe}$ $(0.252 \mathrm{~nm}$ diameter) or the $\mathrm{Pd}$ atoms $(0.274 \mathrm{~nm}$ diameter), mainly bigger atoms were diffused. As is shown in Fig. 16, the increase of the size-dependent activation enthalpy differs in strength for the M-M and Me-M alloys. The slopes are generally smaller for the M-M alloys than for the Me-M alloys. Data are given in Table I on the two Zr-Ti-Cu-Ni-Be bulk glasses V1 and V4 for the su- percooled melt above the caloric glass transition temperature (Geyer et al., 1996; Fielitz et al., 1999; Knorr et al., 2000; Zumkley, Naundorf, et al., 2000). We note that the size dependence in these bulk glasses fits that of the other alloys although the given activation enthalpies have to be regarded as effective quantities because of the structural changes occurring above $T_{g}$ (see Secs. III and IV). The varying slopes for the different amorphous 


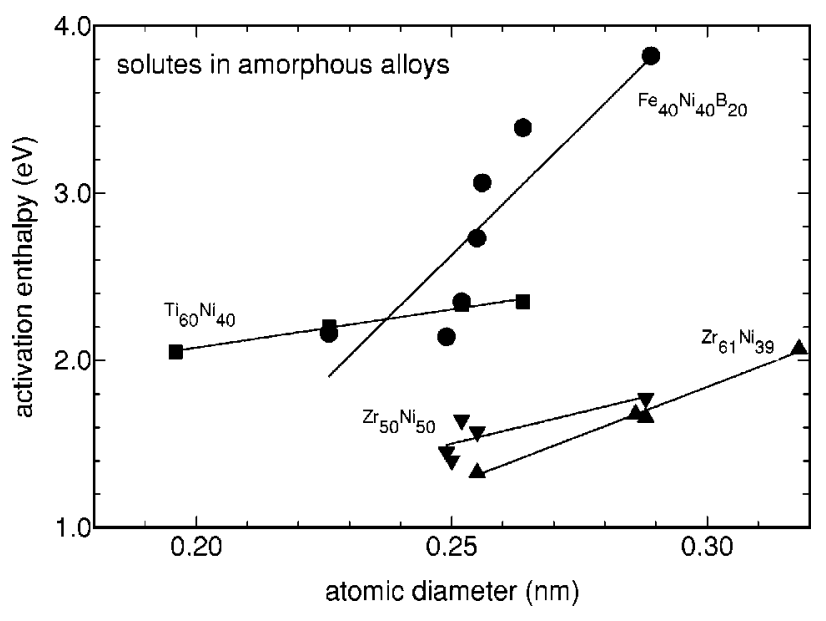

FIG. 16. Size dependence of the activation enthalpy of selfdiffusion and solute diffusion in amorphous $\mathrm{Fe}_{40} \mathrm{Ni}_{40} \mathrm{~B}_{20}$ (solid circles, excluding $\mathrm{Au}$ ), $\mathrm{Ti}_{60} \mathrm{Ni}_{40}$ (solid squares), $\mathrm{Zr}_{61} \mathrm{Ni}_{39}$ (upright solid triangles), and $\mathrm{Zr}_{50} \mathrm{Ni}_{50}$ (reversed solid triangles). Data taken from Table I. Lines are linear least-mean-squares fits to the data.

alloys may indicate a structural influence on the diffusivity. Since the small Be and B atoms tend to densify the structure by filling up the interstices between the larger atoms (backbone atoms), in alloys containing these small atoms diffusion is expected to be much more impeded for the larger solute atoms than for the smaller ones. The size dependence ranges from $31 \mathrm{eV} / \mathrm{nm}$ for $\mathrm{Fe}_{40} \mathrm{Ni}_{40} \mathrm{~B}_{20}$ glass to $4.3 \mathrm{eV} / \mathrm{nm}$ for $\mathrm{Ti}_{60} \mathrm{Ni}_{40}$ glass. It is comparable with that found in $\mathrm{Pb}$, which is about $8 \mathrm{eV} / \mathrm{nm}$ (Fig. 15), and it outweighs by far a slope of $-2.5 \mathrm{eV} / \mathrm{nm}$ observed in crystalline $\mathrm{Ni}$ and $\mathrm{Cu}$.

The basic effects which determine the diffusion mechanism in crystals, elastic and electronic interaction of the solutes with the solvent as mentioned above, do not rely on the periodic structure of crystals. Hence they are also conceivable mechanisms in the disordered structure of the metallic glasses. With this point of view as a working hypothesis, we can understand the size dependence observed in amorphous alloys as the result of a preferentially elastic interaction between the diffusing solute and the surrounding solvent atoms.

If elastic interaction dominates the transport mechanism in amorphous alloys, then we expect that the effective size dependence of the activation enthalpy should be dominated by the quantity $\mu \Omega \delta^{2}$. The solid line in Fig. 17 shows that such a relation describes reasonably well the trend of the size dependence. The data scatter indicates that additional effects that were not taken into account here, for instance, chemical interactions, are of importance and should not be underestimated. Taking into account the data scatter (area between lines in Fig. 17) we derive an average value of $\mu \Omega=(2.6 \pm 1) \mathrm{eV}$. This fitting parameter is in fair agreement with data on the amorphous alloys, for which, using estimated shear moduli $\mu$ (Geschneidner, 1964; Tanner and Ray, 1977; He et al., 1996) and average atomic volumes $\Omega$ (Table I), $\mu \Omega$ values between $3.7 \mathrm{eV}$ (V1 and V4 bulk glasses) and $6 \mathrm{eV}\left(\mathrm{Zr}_{61} \mathrm{Ni}_{39}\right.$ glass $)$ are derived. A similar fit to the size

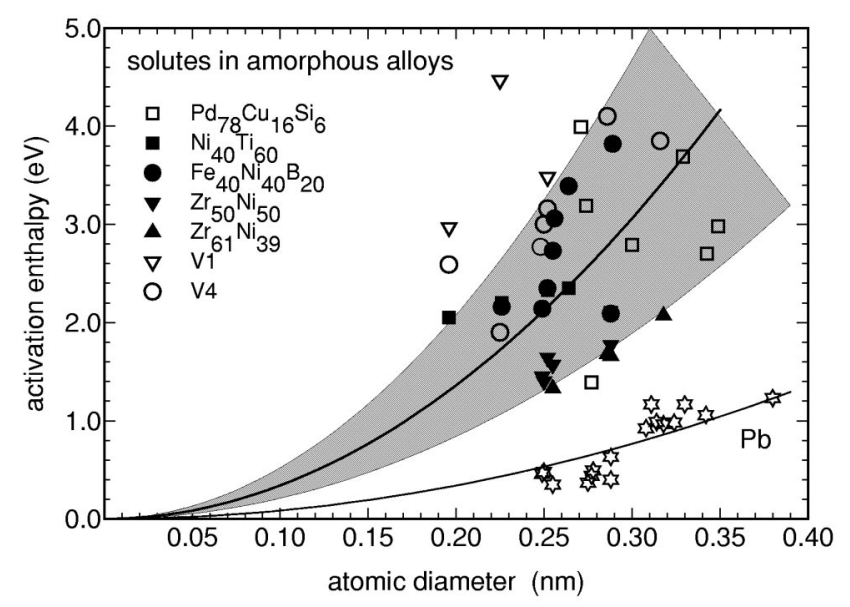

FIG. 17. Fit of the relation $H=\mu \Omega \delta^{2}$ to the size dependence of the solute activation enthalpy in amorphous alloys as taken from Table I. Here $\delta$ is the diameter of the solute normalized to an average diameter of $0.27 \mathrm{~nm}$. Limits of uncertainty for the fitting parameter $\mu \Omega$ are indicated by the hatched area. A fit to the solute diffusion data in $\mathrm{Pb}$ (stars) is also indicated.

dependence in $\mathrm{Pb}$ yields $\mu \Omega=1 \mathrm{eV}$ in agreement with the elastic data. These considerations confirm that the electrostatic interaction between a solute and a vacancytype defect is of minor importance for diffusion in relaxed metallic glasses.

While it appears difficult to identify (static) structural defects in metallic glasses, the dynamic properties of atoms in these disordered structures at diffusion-relevant temperatures can be investigated using moleculardynamics simulations (Sec. IX). These investigations indicate that an important transport mechanism is the migration of atoms by thermally activated displacement chains. Like the dumbbells (diplons) in crystals, these chains interact predominantly elastically with the surrounding matrix; such a mechanism may, at least qualitatively, explain the observed size dependence of the activation enthalpy in metallic glasses.

\section{PRESSURE DEPENDENCE}

\section{A. Introduction}

The question whether diffusion in metallic glasses and undercooled melts is mediated by thermally generated defects - either smeared out or localized — or takes place without thermally generated defects has been widely debated over the years (Cantor, 1986; Averback, 1991; Faupel, 1992; Limoge, 1992a, 1992b; Mehrer, 1996; Frank, 1997; Rätzke et al., 1999). Although the diffusion enthalpies and the preexponential factors of the diffusion coefficients give useful hints as to the diffusion mechanism (Sec. IV), more conclusive information on the role of diffusion vehicles is obtained from the pressure dependence of diffusion at constant temperatures, which may be expressed in terms of an activation volume. Pressure-dependence studies have played a key 
role in elucidating the diffusion mechanisms in crystalline metals (Mehrer, 1990; Philibert, 1991).

Under the assumption that diffusion is thermally activated, with the aid of the thermodynamic relations $G$ $=H-T S$ and $V=(\partial G / \partial p)_{T}$ one can derive the pressure dependence and the activation volume of diffusion. For single-jump diffusion in a crystalline solid, one obtains (Mehrer, 1990)

$$
V_{\text {act }}=-k_{B} T\left[\frac{\partial \ln D}{\partial p}\right]_{T}+k T\left[\frac{\partial \ln \left(f g a^{2} v_{0}\right)}{\partial p}\right]_{T},
$$

where $k_{B}$ is the Boltzmann constant, $T$ the absolute temperature, $D$ the diffusivity, $p$ the pressure, $f$ the correlation factor, $g$ a geometrical factor, $a$ the lattice parameter, and $v_{0}$ the attempt frequency, which is usually of the order of the Debye frequency (see also Secs. III and IV). For amorphous materials suitably averaged values have to be chosen (see below). The second term on the right-hand side of Eq. (6.1) may be simplified. The contribution $\left[\partial \ln v_{0} / \partial p\right]_{T}$ can be estimated using the isothermal compressibility and the Grüneisen constant (see, for example, Mehrer, 1990). It is found to be minor and therefore can be neglected in the case of crystals (Mehrer, 1990).

For a defect-mediated diffusion process in a crystal, the activation volume is composed of a formation term $V^{F}$ and a migration term $V^{M}$ according to

$$
V_{\text {act }}=V^{F}+V^{M} \text {. }
$$

Vacancy-mediated self-diffusion in densely packed metals is characterized by values of $V_{\text {act }}$ between about $0.6 \Omega$ and $1 \Omega$, where, for alloys, $\Omega$ is the average atomic volume (Rätzke and Faupel, 1991; for a data collection see Mehrer, 1990). The main contribution to the activation volume is from the formation volume of vacancies, since calculations and experiments indicate that the migration volume is of the order of $0.1 \Omega$ as far as has been determined (Mehrer, 1990, 1996). For direct interstitial diffusion, which does not involve any formation volume, e.g., $\mathrm{C}$ in $\mathrm{Fe}$, no significant pressure dependence has been observed (Mehrer, 1990, and references therein).

Equation (6.1) was derived for single-jump processes in crystals. In amorphous alloys the quantities $f, g, a$, and $v_{0}$ might be somewhat ambiguous averaged quantities. However, this is not important since only their product enters the second term in Eq. (6.1), which, at least in crystals, is negligible. In particular, the pressure dependence of these quantities should not be too different from that of crystalline metals. Hence there is no fundamental reason why pressure-dependence studies on amorphous alloys should not be evaluated in terms of Eq. (6.1). A vanishing pressure dependence and therefore vanishing activation volume points to a direct diffusion mechanism without thermally generated defects (although, in principle, $V^{F}$ and $V^{M}$ may also compensate each other). On the other hand, a considerable activation volume has been interpreted in terms of thermally generated defects acting as carriers of diffusion (Duine et al., 1994; Klugkist, Rätzke, Rehders, et al., 1998). However, according to recent molecular-dynamics simu-

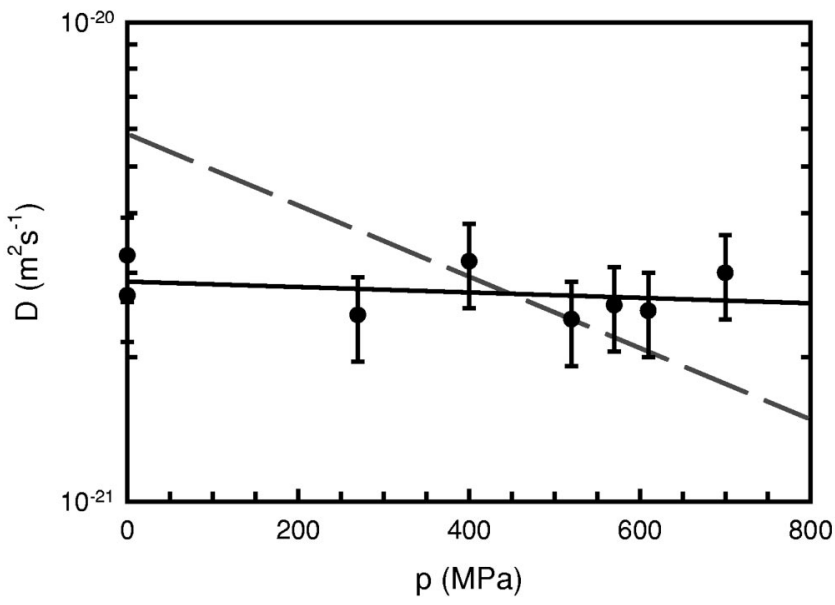

FIG. 18. Isothermal pressure dependence of ${ }^{57} \mathrm{Co}$ diffusion in structurally relaxed amorphous $\mathrm{Co}_{81} \mathrm{Zr}_{19}$ at $563 \mathrm{~K}$. The dashed line corresponds to an activation volume of $1 \Omega$ (Klugkist, Rätzke, Rehders, et al., 1998).

lations (Teichler, 2001; see also Sec. IX), in the case of collective diffusion a significant contribution by the migration volume also has to be considered. Moleculardynamics simulations in amorphous $\mathrm{Ni}_{50} \mathrm{Zr}_{50}$ show that in this alloy the migration volume is approximately $0.43 \Omega$, where $\Omega$ is the average atomic volume.

Diffusivities in samples under various pressures are usually determined by the radiotracer technique described in Sec. II. To avoid mechanical deformation of the sample during diffusion annealing under pressure, hydrostatic pressure is applied with argon as the pressure-transmitting medium. In order to prevent contamination, in particular with oxygen, the gas is highly purified, and, frequently, additional gettering is used, e.g., by wrapping the sample into a $\mathrm{Zr}$ foil or adding a $\mathrm{Zr}$ sponge. Sometimes samples are specially designed with capping layers (Klugkist et al., 1999; Loirat et al., 2000). To avoid changes in the diffusivity due to a systematic temperature drift with increasing pressure, which might be larger than the expected true change of diffusivity caused by the applied pressure, accurate temperature measurement and control within a few $\mathrm{K}$ are necessary. A typical set of data is shown in Fig. 18, where the diffusivity on a logarithmic scale is plotted versus pressure (Klugkist, Rätzke, Rehders, et al., 1998). The activation volume can be calculated from the slope of the line according to Eq. (6.1). In the $\mathrm{Co}_{81} \mathrm{Zr}_{19}$ alloy considered here, the activation volume for Co diffusion is vanishingly small, viz., $V_{\text {act }}=(0.08 \pm 0.1) \Omega$, where $\Omega$ is the average atomic volume of the alloy calculated from the density. An interpretation of this result will be given in Sec. VI.B.1.

\section{B. Data overview}

Despite the experimental difficulties concomitant with the short diffusion length and the strong oxidation tendency of some amorphous alloys, many investigations on the pressure dependence of diffusion in these materials 
TABLE II. Activation volumes of diffusion in metallic glasses (data have been converted to average atomic volumes, where necessary).

\begin{tabular}{|c|c|c|c|c|c|}
\hline No. & Tracer & Matrix & $V_{\text {act }}[\Omega]$ & Remark & Reference \\
\hline 1 & & $\mathrm{Fe}_{40} \mathrm{Ni}_{40} \mathrm{P}_{14} \mathrm{~B}_{6}$ & 1 & $\begin{array}{l}\text { crystallization } \\
\text { kinetics }\end{array}$ & Limoge and Brebec (1988) \\
\hline 2 & ${ }^{60} \mathrm{Co}$ & $\mathrm{Co}_{77} \mathrm{Fe}_{2} \mathrm{Nb}_{14} \mathrm{~B}_{7}$ & $-0.07 \pm 0.1$ & radiotracer & Faupel et al. (1990) \\
\hline 3 & ${ }^{59} \mathrm{Co}$ & $\mathrm{Ni}_{x} \mathrm{Zr}_{1-x}$ & $0.5-1.2$ & $\begin{array}{l}\text { SIMS, } 42<x<62 \text {, } \\
\text { concentration- } \\
\text { dependent }\end{array}$ & Höfler et al. (1992) \\
\hline 4 & $\mathrm{Au}$ & $\mathrm{Pd}_{40} \mathrm{Ni}_{40} \mathrm{~B}_{20}$ & $1 \pm 0.3$ & RBS & Duine et al. (1994) \\
\hline 5 & ${ }^{60} \mathrm{Co}$ & $\mathrm{Fe}_{40} \mathrm{Ni}_{40} \mathrm{~B}_{20}$ & $-0.09 \pm 0.1$ & radiotracer & Rummel and Mehrer (1994) \\
\hline 6 & ${ }^{60} \mathrm{Co}$ & $\mathrm{Fe}_{40} \mathrm{Ni}_{40} \mathrm{~B}_{20}$ & $-0.07 \pm 0.1$ & radiotracer & Rätzke and Faupel (1995) \\
\hline 7 & Hf & $\mathrm{Ni}_{54} \mathrm{Zr}_{46}$ & 0.5 & SIMS & Grandjean et al. (1997) \\
\hline 8 & ${ }^{57} \mathrm{Co}$ & $\mathrm{Co}_{81} \mathrm{Zr}_{19}$ & $0.08 \pm 0.1$ & radiotracer & $\begin{array}{l}\text { Klugkist, Rätzke, Rehders, et al. } \\
\text { (1998) }\end{array}$ \\
\hline 9 & ${ }^{95} \mathrm{Zr}$ & $\mathrm{Co}_{92} \mathrm{Zr}_{8}$ & $0.9 \pm 0.1$ & radiotracer & Klugkist, Rätzke, and Faupel (1998) \\
\hline 10 & $\mathrm{Ni}$ & $\mathrm{Co}_{42} \mathrm{Zr}_{58}$ & $0.66 \pm 0.15$ & SIMS & Klugkist et al. (1999) \\
\hline 11 & ${ }^{63} \mathrm{Ni}$ & $\mathrm{Zr}-\mathrm{Cu}-\mathrm{Ni}-\mathrm{Ti}-\mathrm{Be}$ & $0.35-0.64$ & $\begin{array}{l}\text { radiotracer, } \\
603<T<720 \mathrm{~K}\end{array}$ & Knorr, Macht, and Mehrer $(1999,2000)$ \\
\hline 12 & $\mathrm{Hf}, \mathrm{Au}$ & $\mathrm{Ni}_{54} \mathrm{Zr}_{46}$ & 0.5 & SIMS & Loirat et al. (2000) \\
\hline 13 & $\mathrm{Ni}$ & $\mathrm{Ni}_{50} \mathrm{Zr}_{50}$ & 0.43 & MD simulations & Teichler (2001) \\
\hline
\end{tabular}

have successfully been performed (Table II). At first glance, the results are rather inconsistent and depend on the specific alloy/tracer combination, and therefore are still under discussion. In this section we collect the published data and give a consistent classification of systems as well as some criteria that allow us to differentiate between systems in which the pressure dependence is almost vanishing or considerable.

At first glance, there is no obvious criterion that permits us to interpret the different values obtained in the various systems. The frequently proposed classification with the aid of the activation volumes in diffusion mechanisms operating in metal-metal and metalmetalloid glasses, respectively, obviously fails. It appears to be more useful to differentiate between diffusion mechanisms with respect to the sizes of the tracers and the compositions of the alloys, which is done in the following.

\section{Discussion}

\section{Systems with almost vanishing pressure dependence}

The first direct pressure-dependence measurements were performed on $\mathrm{Co}_{76.7} \mathrm{Fe}_{2} \mathrm{Nb}_{14.3} \mathrm{~B}_{7}$, an alloy that is not prone to oxidation, has a low tendency to crystallization, and thus allows large penetration depths. No pressure dependence of Co diffusion was observed within the error bars, i.e., $V_{\text {act }}=(-0.07 \pm 0.1) \Omega$ (Faupel et al., 1990; Rätzke and Faupel, 1992). This finding rules out diffusion similar to a single-jump vacancy mechanism in crystalline alloys. Under the obvious assumption that in densely packed structures both formation and migration volume are positive quantities, Faupel et al. ruled out that Co diffusion is mediated by thermal defects. Because of the concurrent very small isotope ef- fect in this alloy, it was concluded that diffusion takes place rather by collective rearrangements involving about ten atomic masses (Faupel et al., 1990).

Similar experiments were performed on $\mathrm{Fe}_{40} \mathrm{Ni}_{40} \mathrm{~B}_{20}$, a well-known and well-characterized system. Again, no pressure dependence was found for Co diffusion (Rätzke and Faupel, 1995), and the isotope effect was found to be vanishingly small (Rätzke et al., 1995). This shows that collective diffusion is a quite general feature of metal-metalloid glasses. At the same time, in the Münster group (Rummel and Mehrer, 1994), measurements of ${ }^{60} \mathrm{Co}$ diffusion in $\mathrm{Fe}_{40} \mathrm{Ni}_{40} \mathrm{~B}_{20}$ were performed, and, within the error margins, a pressure dependence was not found either. The excellent agreement of these experiments carried out in different laboratories shows the reliability of the experimental technique.

Finally, the above-mentioned results on the metalmetal glass $\mathrm{Co}_{81} \mathrm{Zr}_{19}$ (Fig. 18), when taken together with the absence of an isotope effect in this system (Heesemann et al., 1995; see also below and Sec. VII), may be interpreted in terms of a direct, collective diffusion mechanism, as were the results on metal-metalloid glasses discussed above.

\section{Systems with considerable pressure dependence}

To compare the self-diffusion behavior of both components in a binary amorphous alloy, $\mathrm{Zr}$ diffusion in $\mathrm{Co}_{92} \mathrm{Zr}_{8}$ was measured (Klugkist, Rätzke, and Faupel, 1998). It was possible to overcome difficulties related to the low specific activity of the ${ }^{95} \mathrm{Zr}$ tracer and the strong oxidation tendency of the alloy. From the pressure dependence of the diffusivities, a large activation volume, $V_{\text {act }}=(0.9 \pm 0.1) \Omega$, was obtained, which was interpreted as being indicative of a defect-mediated diffusion mechanism, in contrast to the direct, collective Co diffu- 
sion mechanism in the $\mathrm{Co}_{81} \mathrm{Zr}_{19}$ alloy (see above). However, the large activation volume of $\mathrm{Zr}$ diffusion does not rule out an indirect, collective diffusion mechanism. For instance, mechanisms via smeared-out defects or chainlike processes are also conceivable. Unfortunately, isotope-effect measurements, which would reveal more details of the diffusion mechanism, appear not to be feasible for $\mathrm{Zr}$ diffusion.

A comparison of the $\mathrm{Zr}$ and Co diffusion coefficients of these two alloys showed that, taking into account the composition dependence, the diffusivity of Co (Heesemann, Rätzke, et al., 2000), exceeds that of $\mathrm{Zr}$ by three to five orders of magnitude in the common temperature regime covered in the experiments. The large activation volume of the $\mathrm{Zr}$ diffusion, as opposed to the vanishing activation volume of Co diffusion, together with the strongly differing diffusivities, suggests that the two constituents migrate via different diffusion mechanisms. This conclusion was also drawn from interdiffusion studies on thin amorphous Ni-Zr-layer samples by Tu and Chou (1988). Amorphous Ni-Zr alloys are known to behave very similarly to $\mathrm{Co}-\mathrm{Zr}$ alloys (Klugkist et al., 1999). These authors observed void formation (opposite to void formation in the classical Kirkendall effect) when both species, the slowly diffusing $\mathrm{Zr}$ and the rapidly diffusing $\mathrm{Ni}$, were mobile. These observations may be considered to support the view that $\mathrm{Zr}$ diffusionunlike Co diffusion-involves thermal defects.

There is evidence from various investigations, including x-ray-scattering experiments, e.g., those of Steyer et al. (1987), electrochemical measurements by Gärtner et al. (1997), and molecular-dynamics simulations (Sec. IX), that the structure and properties of amorphous ( $\mathrm{Ni}$, $\mathrm{Co}, \mathrm{Fe})-\mathrm{Zr}$ alloys change with increasing $\mathrm{Zr}$ concentration. At low $\mathrm{Zr}$ concentrations the structure is relatively loosely packed, with the $\mathrm{Zr}$ atoms filling the low-density regions. At equiatomic concentrations a more densely packed structure with an essentially rigid $\mathrm{Zr}$ backbone is obtained (Rößler and Teichler, 2000). Therefore one would expect that the diffusivity also changes with increasing $\mathrm{Zr}$ content. This has in fact been observed in a systematic study by Heesemann shown in Fig. 19 (Heesemann, Rätzke, et al., 2000; Heesemann, Zöflmer, et al., 2000). These authors observed at a given temperature that the Co diffusivity decreases by several orders of magnitude from $\mathrm{Co}_{90} \mathrm{Zr}_{10}$ to $\mathrm{Co}_{50} \mathrm{Zr}_{50}$. This is in accordance with the molecular-dynamic simulations of Rößler and Teichler (2000). However, the isotope effect of the Co diffusion remained negligibly small over the whole concentration range (Sec. VII). This shows that the diffusion of Co is collective in nature over the whole concentration range.

The interesting question whether there may be a pressure dependence in the case of collective diffusion mechanisms was investigated on nearly equiatomic amorphous Co- $\mathrm{Zr}$ alloys. In order to avoid oxidation of the Zr-rich Co-Zr alloys, a special sample design involving two buried Ni layers was developed, which had the additional advantage that diffusion in both as-quenched and relaxed $\mathrm{Co}_{42} \mathrm{Zr}_{58}$ was simultaneously measurable. $\mathrm{Ni}$

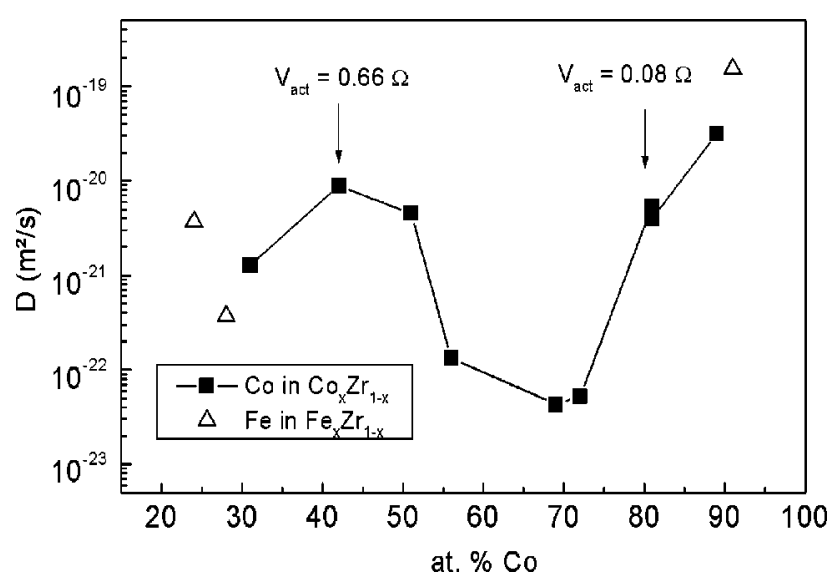

FIG. 19. Isothermal ${ }^{57} \mathrm{Co}$ diffusion coefficient vs Co concentration in structurally relaxed amorphous $\mathrm{Co}-\mathrm{Zr}$ alloys at $573 \mathrm{~K}$ (ם, Heesemann, Zöllmer, et al., 2000; Heesemann, Rätzke, et al., 2000). Only the sample at 69 at. \% Co was in the asquenched state. Data at 89 at. \% and 81 at. \% Co are from Dörner and Mehrer (1991) and Klugkist, Rätzke, Rehders, et al. (1998), respectively. Results for ${ }^{59} \mathrm{Fe}$ diffusion in $\mathrm{Fe}-\mathrm{Zr}$ glasses ( $\triangle$, Horváth et al., 1988) are shown for comparison. Here the concentration refers to Fe. Activation volumes, taken from Klugkist, Rätzke, Rehders, et al., (1998) and Klugkist, Rätzke, Faupel, et al. (1999) are indicated by arrows.

was used as a well-established substitute for probing Co diffusion. Experimental details and the comparison of diffusivities of as-quenched and relaxed glass have been published elsewhere (Klugkist et al., 1999). An important result of this investigation is that $\mathrm{Ni}$ diffusion in relaxed $\mathrm{Co}_{42} \mathrm{Zr}_{58}$ is markedly pressure dependent, i.e., $V_{\text {act }}=(0.66 \pm 0.15) \Omega$, in contrast to the vanishing pressure dependence in Co-rich alloys. However, the absence of an isotope effect suggests that, in spite of this, the diffusion is collective. The relatively small activation enthalpy, about $1.5 \mathrm{eV}$, is in good agreement with activation enthalpies from molecular-dynamics simulation results that were attributed to long-range transport processes (Teichler, 2001).

Since Co and Ni should behave similarly in Co-Zr and $\mathrm{Ni}-\mathrm{Zr}$, these findings may be compared with results of Höfler et al. (1992) on Co diffusion in different amorphous Ni-Zr alloys. These authors found a large, strongly concentration-dependent activation volume for alloys of intermediate $\mathrm{Zr}$ concentration. They interpreted their results in terms of a substitutionalinterstitial-like mechanism similar to that of Co diffusion in crystalline $\alpha$-Zr. Limoge and co-workers determined an activation volume of $V_{\text {act }}=0.5 \Omega$ for $\mathrm{Hf}$ diffusion in amorphous $\mathrm{Ni}_{54} \mathrm{Zr}_{46}$ and also interpreted this result in terms of an interstitial-substitutional mechanism similar to the fast diffusion mechanism in $\alpha-\mathrm{Zr}$ (Grandjean et al., 1997). A classical interstitial-substitutional mechanism, however, is not generally accepted, not even for fast diffusion in $\alpha$ - Zr (Frank, 1989); if this diffusion occurred via a single-jump mechanism into localized defects, it should have an isotope effect of the order of unity, which is in contrast to the vanishing isotope effect observed experimentally (Heesemann, Zöllmer, et al., 
2000). Therefore the diffusion mechanism of $\mathrm{Ni}$ and $\mathrm{Co}$ in equiatomic $\mathrm{Ni}-\mathrm{Zr}$ and $\mathrm{Co}-\mathrm{Zr}$ alloys is expected to be collective in nature.

The preceding discussion was restricted to conventional metallic glasses. Recently, measurements of the pressure dependence of ${ }^{63} \mathrm{Ni}$ diffusion in $\mathrm{Zr}_{47} \mathrm{Ti}_{8} \mathrm{Cu}_{7.5} \mathrm{Ni}_{10} \mathrm{Be}_{27.5}$, a typical representative of the bulk metallic glasses, were performed (Knorr, Macht, and Mehrer, 1999, 2000). The activation volume was found to be in the range of $0.35-0.64 \Omega$ in the supercooled state. As in the case of diffusion in conventional equiatomic $\mathrm{Co}-\mathrm{Zr}$ glasses, the large activation volume was accompanied by a very small Co isotope effect (Ehmler et al., 1998; see also Sec. VII). This supports the view that diffusion in the deeply supercooled state resembles the diffusion in equiatomic $\mathrm{Co}-\mathrm{Zr}$ alloys discussed above, although the interpretation of activation volumes in the supercooled liquid state is complicated by the pressure dependence of the critical temperature $T_{c}$ of the mode-coupling theory. According to molecular-dynamics simulations this effect leads to a considerable and component-dependent increase of the activation volume (Schober, 2002).

In summary, it appears to be useful to classify the activation volumes obtained so far with respect to the size of the tracers and the compositions of the alloys. No pressure dependence is observed if the alloy contains mainly late transition metals such as $\mathrm{Co}$ and $\mathrm{Ni}$ and if the tracer is of the size of the major constituent. On the other hand, a significant pressure dependence is expected if the tracer is not larger than the largest component of the alloy. Diffusion should also be markedly pressure dependent if the alloy has a high concentration of about $50 \%$ of early transition metals like Zr. The experiments of Duine et al. (1994), who found a significant activation volume for $\mathrm{Au}$ diffusion in $\mathrm{Pd}_{40} \mathrm{Ni}_{40} \mathrm{P}_{20}$, and, in particular, the data of Knorr, Macht, and Mehrer, (1999) for $\mathrm{Ni}$ diffusion in $\mathrm{Zr}_{47} \mathrm{Ti}_{8} \mathrm{Cu}_{7.5} \mathrm{Ni}_{10} \mathrm{Be}_{27.5}$ nicely fit in this picture. While the vanishing activation volumes clearly rule out single-jump-type defect-mediated diffusion similar to a vacancy mechanism in crystals, a considerable activation volume must not necessarily be interpreted in terms of thermal defects. The aforementioned molecular-dynamics simulations by Teichler et al. have shown that in the case of thermally activated direct collective mechanisms the migration volume may also be a considerable fraction of an atomic volume. Therefore direct collective diffusion is also consistent with, at least, the smaller (but not negligibly small) measured activation volumes.

\section{ISOTOPE EFFECT}

\section{A. Motivation and definition}

Various features discussed in this article suggest that the diffusion mechanisms in metallic glasses are completely different from the diffusion processes observed in crystalline metals. Rather, they point to collective hopping processes involving a large number of atoms.
This notion is also supported by predictions of the mode-coupling theory (Götze and Sjögren, 1991), which is generally regarded as the most advanced theory of the glass transition at present. According to the modecoupling theory, the cage formed by the neighboring atoms of a given atom freezes in at a critical temperature $T_{c}$ due to the concomitant increase in density. The microscopic glass transition temperature $T_{c}$, which does not depend on the time scale of the experiment, is located well above the caloric glass transition temperature $T_{g}$. As a consequence of this cage effect, liquidlike viscous flow (via atomic collisions) cannot take place below $T_{c}$, and atomic transport (diffusion as well as creep) proceeds exclusively via local hopping processes. These hopping processes, which also occur above $T_{c}$ (Donati et al., 1998), are generally envisaged as being highly collective, i.e., involving many atoms (Sjögren, 1991, see also Sec. IX). The predictions of the mode-coupling theory have been tested in numerous experiments and molecular-dynamics simulations (see, e.g., Sec. IX) on a variety of glass-forming systems, resulting in a qualitative and, in many cases, even quantitative agreement of microscopic dynamics with the theory (Götze, 1999).

In the present section we discuss results from isotopeeffect experiments providing strong evidence that diffusion in the glassy state of metallic glasses takes place by highly collective hopping processes. Isotope-effect measurements in the deeply supercooled liquid state of bulk metallic glasses below $T_{c}$ indicate a similar diffusion behavior.

The isotope effect $E$ for the diffusion of two isotopes with the diffusivities $D_{i}$ and the masses $m_{i}$ is defined as (Schoen, 1958)

$$
E=\left(D_{\alpha} / D_{\beta}-1\right) /\left(\sqrt{m_{\beta} / m_{\alpha}}-1\right) .
$$

For diffusion via single jumps in densely packed lattices, $E$ is generally of the order of unity (Mehrer, 1990) because of the $m^{-1 / 2}$ dependence of the attempt frequency [although strong correlation effects coming into play in special cases, particularly in the diffusion of very diluted impurities that are attracted by vacancies (Philibert, 1991), may give rise to a substantial reduction of $E]$.

In contrast, a highly collective hopping process involving a large number of atoms is expected to cause a very small isotope effect due to the "dilution" of the mass effect by the participation of other atoms in the jump process. A very rough quantitative description of this dilution effect in terms of an effective mass $(m+M)$ of all atoms participating in the collective hopping process is provided by the approach (Mullen, 1961; Le Claire, 1966; Heesemann et al., 1995)

$$
D \propto 1 / \sqrt{m+M} .
$$

The above considerations show that isotope-effect measurements are key experiments to demonstrate the collective nature of diffusion in metallic glasses. Moreover, the isotope effect can be regarded as a "fingerprint" of the diffusion mechanism (Philibert, 1991). Therefore it 


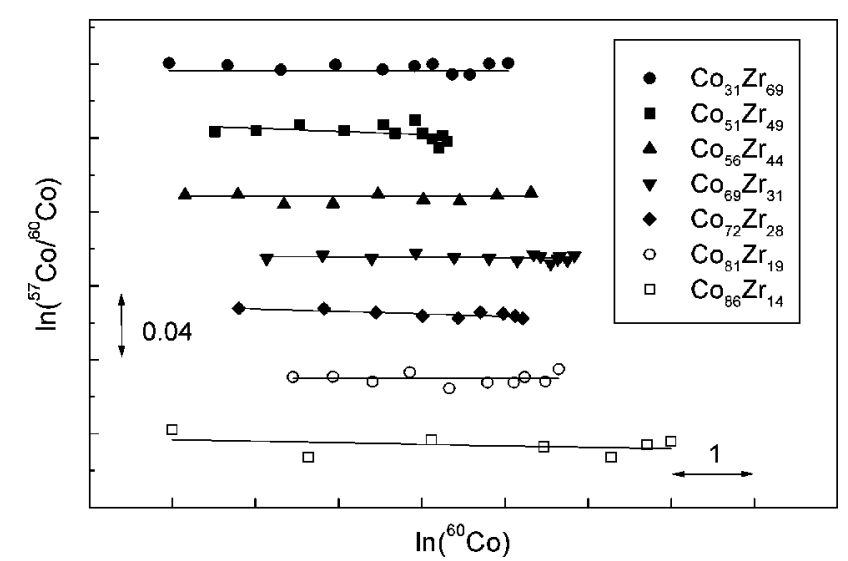

FIG. 20. Isotope-effect profiles of Co diffusion in structurally relaxed amorphous $\mathrm{Co}_{x} \mathrm{Zr}_{1-x}$ alloys at different values of $x$. Only the sample with $x=0.69$ was in the as-quenched state. The radioactivity ratio of ${ }^{57} \mathrm{Co}$ and ${ }^{60} \mathrm{Co}$ is plotted vs the ${ }^{60} \mathrm{Co}$ activity on a logarithmic scale. After Heesemann, Zöllmer, et al., 2000.

can also be used to probe changes in the diffusion mechanism, particularly in connection with the liquid-toglass transition.

\section{B. Diffusion in conventional metallic glasses}

Almost vanishing isotope effects have been observed for Co diffusion in various metallic glasses including metal-metalloid systems (Faupel et al., 1990; Hüppe and Faupel, 1992; Heesemann et al., 1995; Rätzke et al., 1995; Heesemann, Zöllmer, et al., 2000). E was determined by measuring the simultaneous diffusion of the radiotracers ${ }^{57} \mathrm{Co}$ and ${ }^{60} \mathrm{Co}$, employing the serial sectioning technique in conjunction with ion-beam sputtering. Resulting isotope-effect profiles such as those depicted in Fig. 20 were evaluated by means of Eq. (7.3), which was obtained by a simple rearrangement of the thin-film solution of Fick's second law [Eq. (2.2)],

$$
\ln \frac{C_{\alpha}}{C_{\beta}}=\text { const }^{\prime}-\left(\frac{D_{\beta}}{D_{\alpha}}-1\right) \ln C_{\beta} .
$$

Here, $C_{i}$ is the concentration of isotope $i$. Isotope effects were calculated from the slopes of linear fits to the isotope-effect profiles based on Eqs. (7.1) and (7.3). Figure 21 summarizes the isotope-effect results in conventional metallic glasses.

The essentially vanishing isotope effects measured in metallic glasses were taken as strong evidence for a highly collective hopping mechanism, as suggested by the above-mentioned theoretical considerations and molecular-dynamics simulations (Sec. IX; Oligschleger and Schober, 1999; Teichler, 2001). In these terms, the extremely small isotope effects were attributed to a strong dilution of the mass dependence of diffusion due to the participation of a large number of atoms in collective hopping processes. In molecular-dynamics simulations mainly chainlike displacements have been observed. A typical example is displayed in Fig. 28 (Sec.

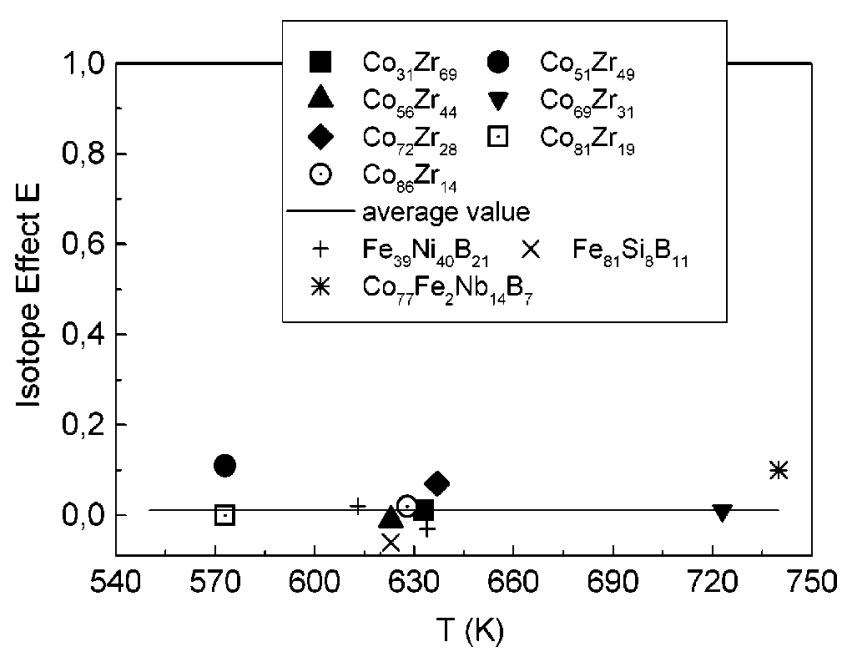

FIG. 21. Summary of results from isotope-effect measurements of Co diffusion in conventional metallic glasses. One notes that all isotope effects are very small and generally very close to zero. The data were taken from Hüppe and Faupel (1992; $\mathrm{Co}_{76.7} \mathrm{Fe}_{2} \mathrm{Nb}_{14.3} \mathrm{~B}_{7}$ ), Heesemann et al. (1995; Co-Zr), Heesemann, Zöllmer, et al. (2000; Co-Zr), and Rätzke et al. (1995; Fe-Ni-B, Fe-Si-B).

IX). The computer simulations suggest that the number of atoms participating in a collective hopping process is very high, typically well above ten (Schober et al., 1997; Oligschleger and Schober, 1999; Teichler, 2001). Similarly high numbers result from isotope-effect measurements via Eqs. (7.1) and (7.2) (Heesemann et al., 1995; Heesemann, Zöllmer, et al., 2000). As discussed in Sec. IX, computer simulations also indicate a connection between the low-frequency excitations in glasses and highly collective long-range diffusion (Schober et al., 1997; Oligschleger and Schober, 1999). We emphasize, however, that the isotope-effect measurements do not provide an atomistic picture of the jump process and hence do not rule out other collective mechanisms, e.g., those involving smeared-out defects. In this context we refer the reader to the discussion in Sec. VI of large activation volumes in conjunction with very low isotope effects (see Figs. 19 and 21 as well as Fig. 1 in Heesemann, Zöllmer, et al., 2000).

Mechanisms based on delocalized thermal defects were proposed previously (Spaepen, 1981; Stelter and Lazarus, 1987; Averback, 1991; van den Beukel, 1993). A well-known example of the notion of delocalized defects is the spread-out "free" volume within the freevolume approach, which was originally applied to metallic glasses by Spaepen (1981) and has recently been revived based on results from a novel nuclear magnetic resonance technique (Tang et al., 1998).

So far, we have discussed isotope effects in structurally relaxed metallic glasses. Only in these systems is the structure stable during a diffusion experiment and can reproducible diffusion measurements be done. On the other hand, unrelaxed conventional metallic glasses as well as bulk glasses (Nagel et al., 1998, 1999) contain excess free volume quenched-in from the liquid state (Sec. III). This excess volume affects nearly all proper- 


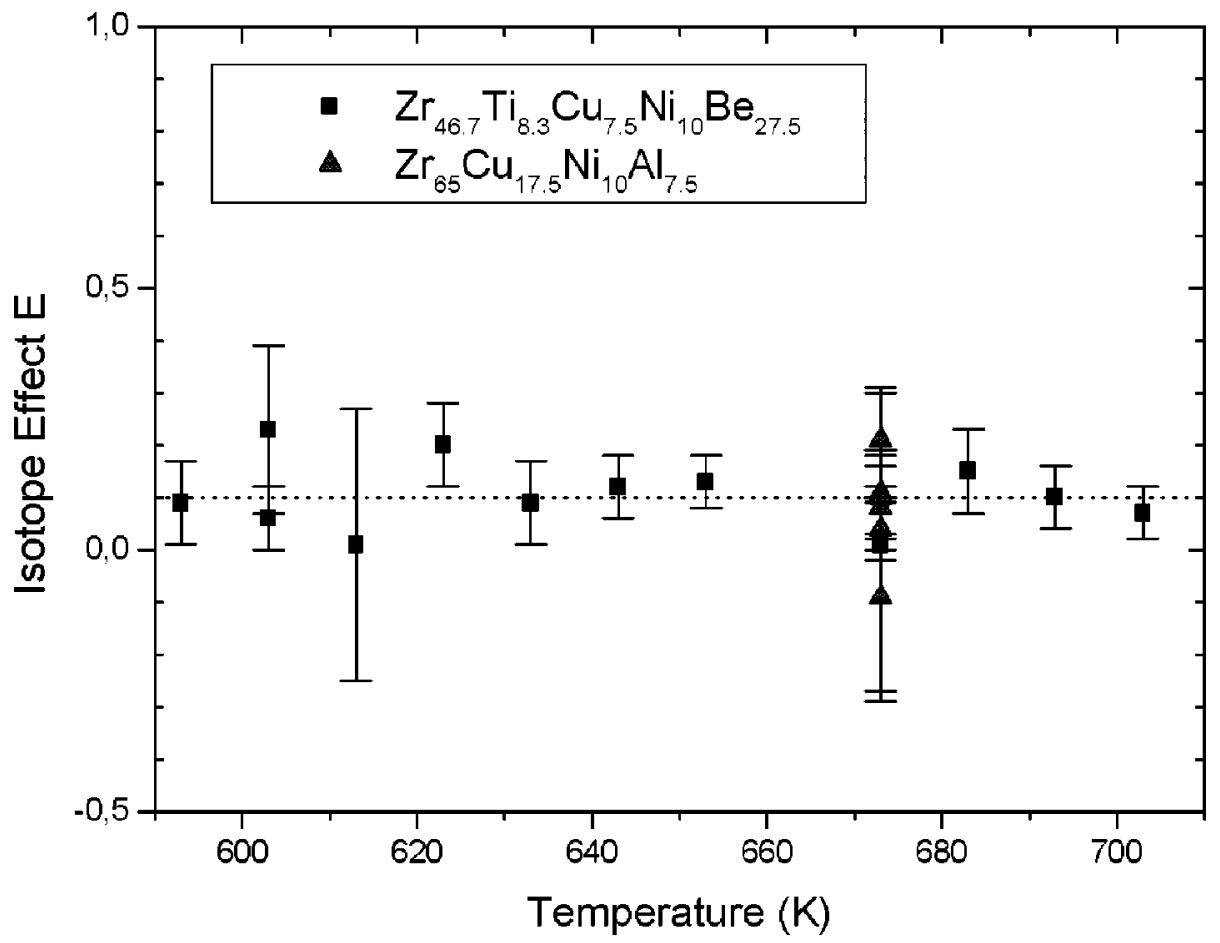

FIG. 22. Isotope effect as a function of temperature for $\mathrm{Co}$ diffusion in bulk metallic glasses. The data were taken from Ehmler et al. (1998, 1999).

ties, and, for instance, annealing of excess volume during irreversible structural relaxation may lead to severe embrittlement (Egami, 1981).

It is obvious that excess free volume also enhances diffusion (see Sec. III and Horváth et al., 1987; Zöllmer et al., 2000). Therefore we have investigated the influence of excess volume on the diffusion mechanism. Significant isotope effects in the as-quenched metalmetalloid glass $\mathrm{Co}_{76.7} \mathrm{Fe}_{2} \mathrm{Nb}_{14.3} \mathrm{~B}_{7}$ (Rätzke et al., 1992) lend support to the notion of quenched-in quasivacancies, which migrate to the sample surface during structural relaxation (Rätzke et al., 1992). Alternatively, our recent isotope-effect measurements in thin amorphous $\mathrm{Co}_{51} \mathrm{Zr}_{49}$ films do not show any change in the diffusion mechanism during structural relaxation and point to a collective hopping mechanism in the relaxed as well as in the as-quenched state (Zöllmer et al., 2000; Heesemann et al., 2001). As mentioned in Sec. III, in these $\mathrm{Co}-\mathrm{Zr}$ films excess volume appears to annihilate intrinsically, e.g., by recombination of regions of higher and lower density on the nanoscopic scale, as first suggested by Egami (1981). A more detailed explanation is given in Sec. VIII (see, in particular, footnote 5).

\section{Diffusion in the deeply supercooled liquid state of bulk metallic glasses}

Recently, isotope-effect measurements involving the isotopes ${ }^{57} \mathrm{Co}$ and ${ }^{60} \mathrm{Co}$ have also been carried out by means of the radiotracer technique in the deeply supercooled liquid state of bulk metallic glasses. The results are displayed in Fig. 22. For experimental details, the reader is referred to Ehmler et al. (1998, 1999).

One notes that the isotope effect is very small over the whole temperature range and exhibits no significant temperature dependence. The magnitude of the isotope effect is similar to that of the isotope effects found in the glassy state of conventional metallic glasses (Fig. 21). This lends strong support to the view that the collective nature of the diffusion mechanisms does not change at the caloric glass transition temperature and that highly collective hopping processes occurring in the glassy state still determine long-range diffusion in a deeply supercooled melt. Collective hopping of many atoms has also been observed in deeply supercooled melts in moleculardynamics simulations (see Sec. IX and Schober et al., 1997). The order of magnitude of ten atoms participating in the hopping processes is in agreement with the measured $E$ values (Ehmler et al., 1998, 1999). Very recent measurements of the isotope effect in $\mathrm{Pd}_{40} \mathrm{Cu}_{30} \mathrm{Ni}_{10} \mathrm{P}_{20}$ glasses confirm the view that the diffusion mechanism does not change near $T_{g}$ (Zöllmer et al., 2001): $E$ was measured at the same temperature near $T_{g}$ in the glassy state and, after long-time relaxation, in the supercooled liquid state. While the diffusivity dropped by about an order of magnitude, no change in $E$ was observed. $E$ turned out to be very low $(\approx 0.1)$ up to temperatures near $T_{c}$, supporting the notion of collective hopping.

We point out that the isotope effects measured in the deeply supercooled melt state of bulk metallic glasses are far too low to be compatible with ordinary viscous flow in the hydrodynamic regime. Here kinetic theories predict (Nachtrieb, 1976; Kirkaldy and Young, 1987)

$$
D \propto \frac{1}{\sqrt{m}} T^{n},
$$

where $n$ is close to 2 according to molecular-dynamics simulations (Shimoji and Itami, 1986). Equations (7.1) and (7.4) yield an isotope effect of unity. Isotope-effect 
measurements of the self-diffusion in liquid Sn far above the melting point $T_{m}$ have indeed revealed an $E$ value of the order of unity, confirming the likelihood of essentially uncorrelated single-particle motion (Frohberg et al., 1987). Upon lowering the temperature of the melt, the isotope effect was found to decrease from $E=0.7$ at $568 \mathrm{~K}$ to about 0.5 near $T_{m}=505 \mathrm{~K}$, indicating deviations from uncorrelated single-particle motion already in the ordinary liquid near the melting point. Recent molecular-dynamics simulations on diffusion in a simple liquid are in accord with these experiments and the predictions from kinetic theories (see Sec. IX and Schober, 2001). Based on the low values of the isotope effect we can hence definitely rule out ordinary viscous flow in the deeply supercooled liquid state.

Apparently, the diffusion behavior in a deeply supercooled melt is very similar to that of diffusion in the glassy state, as expected from the theoretical concepts discussed above, which rule out a change in the nature of the diffusion mechanisms at the caloric glass transition temperature. The present picture of thermally activated collective hopping processes governing diffusion in the glassy as well as in the deeply supercooled liquid state is in sharp conflict with the aforementioned previous interpretations of the kinks in Arrhenius plots in terms of a basic change in the diffusion mechanism (see Secs. I, III, and IV; Greer, 1999; Tang et al., 1999).

\section{DIFFUSION UNDER IRRADIATION}

For crystalline solids, studies of diffusion under particle irradiation have proven to be a powerful tool in elucidating diffusion mechanisms. Therefore it is not surprising that, after the advent of metallic glasses, several diffusion-under-irradiation experiments were done on these materials, too. Before presenting these experiments, we provide a theoretical basis for their interpretation.

\section{A. Theoretical considerations}

There is experimental evidence that in metallic glasses there are regions resembling defects in crystalline solids. An example is the excess free volume in unrelaxed metallic glasses (Sec. III), which exhibits the features of a supersaturation of "vacancies." This picture will be extended by postulating that, in addition to such "quasivacancies" as defined in Sec. III, metallic glasses may contain other "quasidefects" as well. (For details, see Sec. VIII.C.1.)

Diffusion in solids can be influenced by particle irradiation via a variety of mechanisms (Frank et al., 1980). We shall not list all of these here. Rather we restrict ourselves to the discussion of two mechanisms, in terms of which all experimental findings on diffusion in metallic glasses under particle irradiation can be rationalized.

Mechanism (i) (Sec. VIII.A.1) was originally invoked for crystalline solids (Sizmann, 1978). It is based on the assumption that irradiation may introduce mobile selfdefects that act as diffusion vehicles and thus may give rise to diffusion enhancement. This mechanism leads to a natural explanation of enhanced diffusion in metallic glasses under high-transferred-energy irradiations (Sec. VIII.B.1).

Mechanism (ii) (Sec. VIII.A.2) evolved from previous proposals (Schuler et al., 1997, 1999; Schuler, 2001) that aimed at accounting for a novel type of irradiationenhanced diffusion (enhanced diffusivity at unchanged diffusion enthalpy), which was observed in metallic glasses under low-transferred-energy irradiations (Sec. VIII.B.2). The basic assumption underlying this mechanism is that radiation may alter the structure of a solid and thus the inherent diffusion mechanism. In general, this gives rise to a change in diffusivity.

1. Diffusion enhancement by radiation-produced mobile selfdefects [mechanism (i)]

The extra diffusivity $\Delta D^{\text {irr }}$ arising from radiationproduced vacancies and interstitials has been calculated within the framework of a rate-equation approach by Sizmann (1978). Allowing for vacancy-interstitial recombination as well as for annihilation of both defect species at unsaturable sinks, Sizmann's theory predicts that constant-flux irradiation finally results in a steady state in which $\Delta D^{\text {irr }}$ is controlled by an activation enthalpy $H^{\text {irr }}$ ranging from $H_{s}^{M} / 2$ in the recombination-controlled case to zero in the sink-controlled case. Here $H_{s}^{M}$ is the migration enthalpy of the slower defect species. Whereas in the case controlled by recombination $\Delta D^{\text {irr }}$ depends on the irradiation-particle flux $\dot{\Phi}$ according to $\Delta D^{\text {irr }} \sim \dot{\Phi}^{1 / 2}$, in the sink-controlled case $\Delta D^{\text {irr }} \sim \dot{\Phi}$. Moreover, $H^{\mathrm{irr}} \geqslant H$ (diffusion enthalpy in the absence of irradiation) precludes the mediation of diffusion by the slower defects. That is, in this case, diffusion without irradiation takes place either via the faster defect species or by a direct diffusion mechanism. Finally it is emphasized that according to Sizmann's theory $H^{\text {irr }}=H$ may occur by chance, but not due to a special physical situation.

\section{Diffusivity alterations via radiation-induced structural changes [mechanism (ii)]}

The mechanisms controlling diffusion in a solid may be altered if the structure is changed by irradiation. This is of particular interest if, even in the absence of irradiation, diffusion is confined to a network-shaped phase (henceforth called a $\delta$ phase) that is embedded in the matrix. Such a mechanism may operate if the diffusion in the $\delta$ phase is distinctly faster than in the matrix and the percolation limit of the network is exceeded. Then, in simple cases, the diffusion coefficient $D_{\mathrm{R}}$ obeys an Arrhenius law,

$$
D_{\mathrm{R}}=D_{0} \exp (-H / k T)
$$

whose preexponential factor $D_{0}$ is proportional to the volume fraction of the $\delta$ phase. The subscript " $\mathrm{R}$ " indi- 
cates that relaxed metallic glasses are considered, which do not contain vacancies in supersaturation (Secs. III, VIII.C.1, and VIII.C.2).

Provided that irradiation transforms matrix phase into $\delta$ phase, irradiation leads to an increase in the preexponential factor, but leaves the activation enthalpy unchanged. By this mechanism a steady state of radiationenhanced diffusion can be achieved if, in contrast to the frozen-in network-shaped $\delta$ phase, the extra $\delta$ phase produced by irradiation consists of small spots which comprise only a few atoms and retransform spontaneously into the matrix phase with a half-life $\tau$ being at least comparable with the mean dwelling time of the diffusing atoms. As shown by Scharwaechter et al. (1996b), under these circumstances

$$
\Delta D^{\mathrm{irr}}=\left(\sigma \tau \dot{\Phi} / F_{0}\right) D_{\mathrm{R}}=\left(\sigma \tau \dot{\Phi} / F_{0}\right) D_{0} \exp (-H / k T),
$$

where $\sigma$ is the cross section for the production of $\delta$ spots and $F_{0}$ the volume fraction of the $\delta$ phase prior to irradiation.

A suggested atomic structure of the $\delta$ phase will be given in Sec. VIII.C.1.

\section{B. Experiments and their interpretations}

\section{Diffusion under high-transferred-energy irradiation}

In 1987 B $\varnothing$ ttiger, Pampus, and Torp reported on Rutherford backscattering studies that showed enhanced diffusion of implanted $\mathrm{Au}$ in amorphous $\mathrm{Pd}_{78} \mathrm{Cu}_{6} \mathrm{Si}_{16}$ under $500 \mathrm{keV}-\mathrm{Xe}^{+}$irradiation and $200 \mathrm{keV}-\mathrm{Ne}^{+}$irradiations. Since the data were taken only at a few temperatures, they do not allow us to draw reliable conclusions.

As early as 1986, Averback, Hahn, and collaborators (Hahn et al., 1986, 1987; Averback and Hahn, 1988, 1989; Averback et al., 1988) published several studies of diffusion under $1 \mathrm{MeV}-\mathrm{Kr}^{+}$irradiation in amorphous $\mathrm{Ni}-\mathrm{Zr}$, Ni-Ti, and $\mathrm{Cu}-\mathrm{Er}$ alloys. The measuring techniques they used were mainly Rutherford backscattering spectroscopy and secondary-ion mass spectroscopy. Their most extensive studies concerned the diffusion of $\mathrm{Cu}$ and $\mathrm{Au}$ in amorphous $\mathrm{Ni}_{50} \mathrm{Zr}_{50}$ or $\mathrm{Ni}_{45} \mathrm{Zr}_{55}$. These cover the temperature range $80-620 \mathrm{~K}$. In the absence of irradiation, $\mathrm{Cu}$ diffuses in amorphous $\mathrm{Ni}_{50} \mathrm{Zr}_{50}$ much faster than $\mathrm{Au} \quad\left(\mathrm{Cu}: \quad H=1.57 \mathrm{eV}, \quad D_{0}=1.8\right.$ $\times 10^{-7} \mathrm{~m}^{2} \mathrm{~s}^{-1}$; Au: $H=1.7 \mathrm{eV}, D_{0}=1.5 \times 10^{-8} \mathrm{~m}^{2} \mathrm{~s}^{-1}$ ). Under $\mathrm{Kr}^{+}$irradiation this remains true, though the diffusivities are enhanced considerably. Below about $400 \mathrm{~K}$ for $\mathrm{Cu}$ and $500 \mathrm{~K}$ for $\mathrm{Au}$, the radiation enhancement is temperature independent and therefore, with great likelihood, due to ion-beam mixing, i.e., a redistribution of the matrix atoms in the displacement cascades as a result of momentum transfers from the irradiation particles (Müller et al., 1988). At higher temperatures, $\Delta D^{\text {irr }}$ becomes temperature dependent. In this regime of true radiation-enhanced diffusion, Averback and Hahn found for $\mathrm{Cu}$ in $\mathrm{Ni}_{45} \mathrm{Zr}_{55}$ a $\dot{\Phi}^{1 / 2}$ dependence of $\Delta D^{\text {irr }}(523 \mathrm{~K})$ and an activation enthalpy $H^{\mathrm{irr}} \approx 0.55 \mathrm{eV}$. This rules out mechanism (ii) [Eq. (8.2)], but is in accordance with the recombination-controlled case of mechanism (i). Because of $H^{\mathrm{irr}}(=0.55 \mathrm{eV})<H(=1.57 \mathrm{eV})$, one cannot extract any information on the diffusion mechanism in the absence of irradiation. It may be concluded that the activation enthalpy of the slower defect species is $H_{s}^{M}$ $\equiv 2 H^{\mathrm{irr}} \approx 1.1 \mathrm{eV}$, but it cannot be decided whether this defect species is of vacancy or of interstitial type.

In 1991 Tyagi, Macht, and Naundorf (1991a) reported on secondary-ion-mass-spectroscopy measurements of the self-diffusion of ${ }^{63} \mathrm{Ni}$ in amorphous $\mathrm{Fe}_{40} \mathrm{Ni}_{40} \mathrm{~B}_{20}$ under $300 \mathrm{keV}-\mathrm{Ni}^{+}$irradiation at temperatures between 294 and $582 \mathrm{~K}$ and $\mathrm{Ni}^{+}$fluxes ranging from 1 to $10 \mathrm{~mA} / \mathrm{m}^{2}$. Below $500 \mathrm{~K}$ they observed a radiationenhanced mass transport, which, as indicated by its temperature independence and its proportionality to the $\mathrm{Ni}^{+}$flux, arose from ion-beam mixing. Above about $500 \mathrm{~K}$, thermally activated radiation-enhanced diffusion occurred with an activation enthalpy $H^{\mathrm{irr}}=0.65 \mathrm{eV}$ and $\Delta D^{\text {irr }} \sim \Phi^{0.7}$. Since in the absence of irradiation ${ }^{63} \mathrm{Ni}$ selfdiffusion in amorphous $\mathrm{Fe}_{40} \mathrm{Ni}_{40} \mathrm{~B}_{20}$ is controlled by the activation enthalpy $H=(2.14 \pm 0.16) \mathrm{eV}$ (Tyagi et al., 1991b), as in the Averback-Hahn experiments $H^{\text {irr }}$ is smaller than $H$. While these data exclude mechanism (ii), they show the features of diffusion enhancement by radiation-produced mobile self-defects [mechanism (i)]. However, again these data neither reveal the nature of the diffusion mechanism in the absence of irradiation nor allow us to decide whether the vacancy or the interstitial is the slower radiation-produced defect species. The $\Delta D^{\text {irr }} \sim \dot{\Phi}^{0.7}$ dependence signals an intermediate situation between the recombination-controlled $\left(\Delta D^{\text {irr }}\right.$ $\sim \Phi^{1 / 2}$ ) and the sink-controlled $\left(\Delta D^{\mathrm{irr}} \sim \Phi\right)$ cases (Sizmann, 1978). Therefore one may conclude that $H_{s}^{M}$ is larger than $2 H^{\text {irr }}=1.3 \mathrm{eV}$.

The above-described diffusion-under-irradiation experiments have in common that the energies transferred to the matrix atoms lie well above the threshold energies for atomic displacements. This is confirmed by the occurrence of ion-beam mixing. Under these conditions, part of the radiation damage presumably consists of nonclustered vacancies and self-interstitials that at elevated temperatures become mobile and serve as vehicles for enhanced diffusion. In this respect, metallic glasses behave very similarly to crystalline metals. Thus the transfer of the defect concept from crystalline solids to metallic glasses is justified posteriorly.

\section{Diffusion under low-transferred-energy irradiation}

Since 1993 Frank and co-workers (Scharwaechter, 1993; Frank et al., 1996; Scharwaechter et al., 1996b; Schuler et al., 1997, 1999; Schuler, 2001) have published a series of papers on self-diffusion in relaxed melt-spun metallic glasses under irradiation with $0.4 \mathrm{MeV}$ protons, $0.5 \mathrm{MeV}$ protons, $1 \mathrm{MeV}-\mathrm{He}^{+}$ions, or $1.4 \mathrm{MeV}-{ }^{15} \mathrm{~N}^{+}$ ions. They used the radiotracer technique in combination with ion-beam sputtering for serial sectioning of the specimens. During diffusion under irradiation a partial area of the specimens was shielded from irradiation, thus permitting a simultaneous investigation of diffusion 


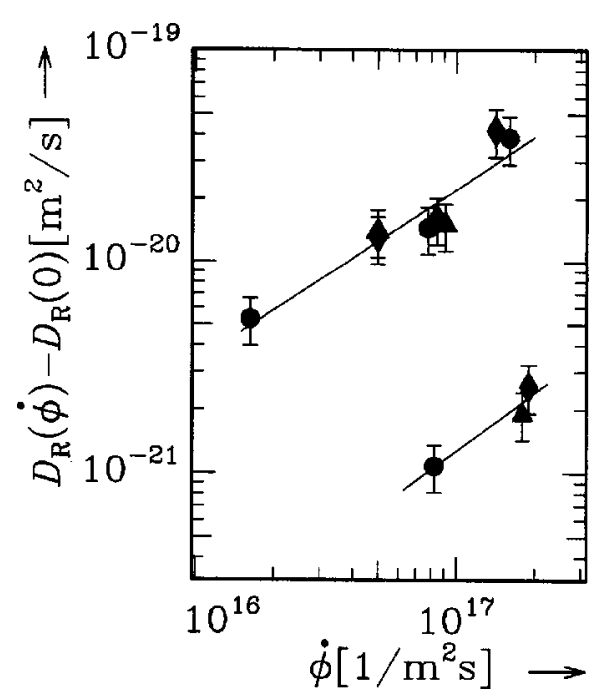

FIG. 23. Enhancement of the ${ }^{59} \mathrm{Fe}$ diffusion coefficient in relaxed amorphous $\mathrm{Co}_{58} \mathrm{Fe}_{5} \mathrm{Ni}_{10} \mathrm{Si}_{11} \mathrm{~B}_{16}$ as a function of the proton flux at $380{ }^{\circ} \mathrm{C}$ (lower data group) and $420{ }^{\circ} \mathrm{C}$ (upper data group), respectively. After Scharwaechter et al., 1996b.

under and in the absence of irradiation, on the same specimen, under otherwise identical conditions.

The first investigations of this kind dealt with selfdiffusion of ${ }^{59} \mathrm{Fe}$ in $\mathrm{Co}_{58} \mathrm{Fe}_{5} \mathrm{Ni}_{10} \mathrm{Si}_{11} \mathrm{~B}_{16}$ under $0.4 \mathrm{MeV}$ proton irradiation. The experiments made sure that $\Delta D^{\text {irr }}$ was measured under steady-state conditions. For instance, during diffusion annealing at $420^{\circ} \mathrm{C}$ under a flux of $8 \times 10^{16}$ protons $/ \mathrm{m}^{2} \mathrm{~s}$, a steady state evolved after about $1 \mathrm{~h}$. Figure 23 shows that, within the error bars, $\Delta D^{\text {irr }}$ is proportional to $\dot{\Phi}$, which is in contrast to $\Delta D^{\mathrm{irr}} \sim \dot{\Phi}^{1 / 2}$ and $\Delta D^{\mathrm{irr}} \sim \dot{\Phi}^{0.7}$ reported for diffusion under high-transferred-energy irradiations with $\mathrm{Kr}^{+}$and $\mathrm{Ni}^{+}$ions, respectively (Sec. VIII.B.1).

The most surprising result of the studies of ${ }^{59} \mathrm{Fe}$ diffusion in $\mathrm{Co}_{58} \mathrm{Fe}_{5} \mathrm{Ni}_{10} \mathrm{Si}_{11} \mathrm{~B}_{16}$ under $0.4 \mathrm{MeV}-\mathrm{H}^{+}$irradiation concerns the temperature dependence (Fig. 24). There is a distinct radiation enhancement $\Delta D^{\mathrm{irr}}$ which is exclusively due to an increase in the preexponential factor in the Arrhenius law of the diffusion coefficient, whereas the diffusion enthalpies under irradiation and in the absence of irradiation are the same, viz., $H^{\text {irr }}=H$ $(=2.25 \mathrm{eV})$. This finding is not compatible with mechanism (i), i.e., radiation enhancement owing to diffusionmediating mobile self-defects produced by irradiation may be excluded. It is true that in the case of mechanism (i) $H^{\text {irr }}$ may be equal to $H$ by chance (Sec. VIII.A.1); however, this cannot happen for $\Delta D^{\mathrm{irr}} \sim \dot{\Phi}$ since, according to Sizmann's theory (1978), this flux dependence is only compatible with $H^{\mathrm{irr}}=0$. On the other hand, within the framework of mechanism (ii), $H^{\text {irr }}=H$ and $\Delta D^{\text {irr }}$ $\sim \dot{\Phi}$ find a natural reconciliation if, in the absence of irradiation, diffusion is confined to a frozen-in network of a $\delta$ phase that is embedded in the matrix and, under irradiation, small $\delta$ spots of finite lifetime are introduced [Eq. (8.2)].

The reason for the enhancement of ${ }^{59} \mathrm{Fe}$ diffusion in irradiated $\mathrm{Co}_{58} \mathrm{Fe}_{5} \mathrm{Ni}_{10} \mathrm{Si}_{11} \mathrm{~B}_{16}$ by the type-(ii) mecha-

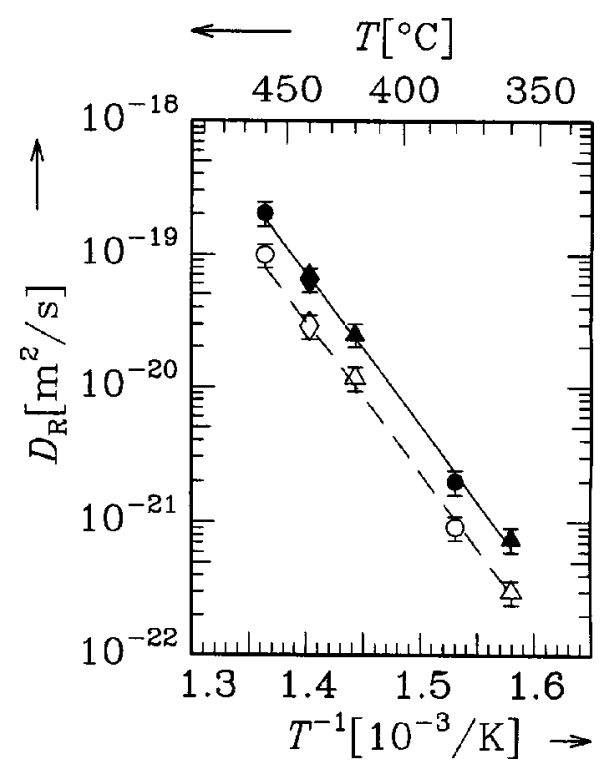

FIG. 24. Temperature dependence of the diffusion coefficient of ${ }^{59} \mathrm{Fe}$ in relaxed amorphous $\mathrm{Co}_{58} \mathrm{Fe}_{5} \mathrm{Ni}_{10} \mathrm{Si}_{11} \mathrm{~B}_{16}$ : filled symbols, specimen sections exposed to irradiation with about 1.3 $\times 10^{17}$ protons $/ \mathrm{m}^{2} \mathrm{~s}$; open symbols, specimen sections shielded from irradiation. After Scharwaechter et al., $1996 \mathrm{~b}$.

nism just described may be the different energies transferred by proton irradiation and heavy-ion irradiation. [By contrast, in the experiments of Averback, Hahn, and co-workers and of Tyagi, Macht, and Naundorf, type-(i) mechanisms operate.] If this is the case the threshold energy for the production of distant Frenkel-type pairs, whose vacancy and self-interstitial analog can undergo long-range migration, must be higher than that for $\delta$-spot production. Then a decrease of the transferred energy in diffusion-under-irradiation experiments with a given diffuser in a given matrix must lead to a transition in the radiation-enhanced diffusion mechanism from type (i) to type (ii) and should be reflected by an increase in the activation enthalpy from $H^{\text {irr }}<H$ to $H^{\text {irr }}$ $=H$. This prediction has been checked in diffusionunder-irradiation studies of ${ }^{59} \mathrm{Fe}$ in relaxed glassy $\mathrm{Fe}_{91} \mathrm{Zr}_{9}$ (Fig. 25). Indeed, with decreasing irradiation energy $\left(1.4 \mathrm{MeV}-{ }^{15} \mathrm{~N}^{+}\right.$irradiation, $1 \mathrm{MeV}-\mathrm{He}^{+}$irradiation, $0.5 \mathrm{MeV}-\mathrm{H}^{+}$irradiation), $H^{\text {irr }}$ increases and becomes equal to $H$ under irradiation with $0.5 \mathrm{MeV}$ protons. For the subsequent discussion it is important to note that, in contrast to what is true for ${ }^{59} \mathrm{Fe}$ in $\mathrm{Fe}_{91} \mathrm{Zr}_{9}$, the diffusion of ${ }^{95} \mathrm{Zr}$ in relaxed amorphous $\mathrm{Fe}_{24} \mathrm{Zr}_{76}$ is not enhanced by $0.5 \mathrm{MeV}-\mathrm{H}^{+}$irradiation (Schuler et al., 1999; Schuler, 2001).

\section{Facts and speculations}

In this section, an admittedly speculative view of defects and diffusion mechanisms in conventional and bulk metallic glasses is presented which accounts for a large body of experimental findings in a natural way (Frank et al., 2003). 


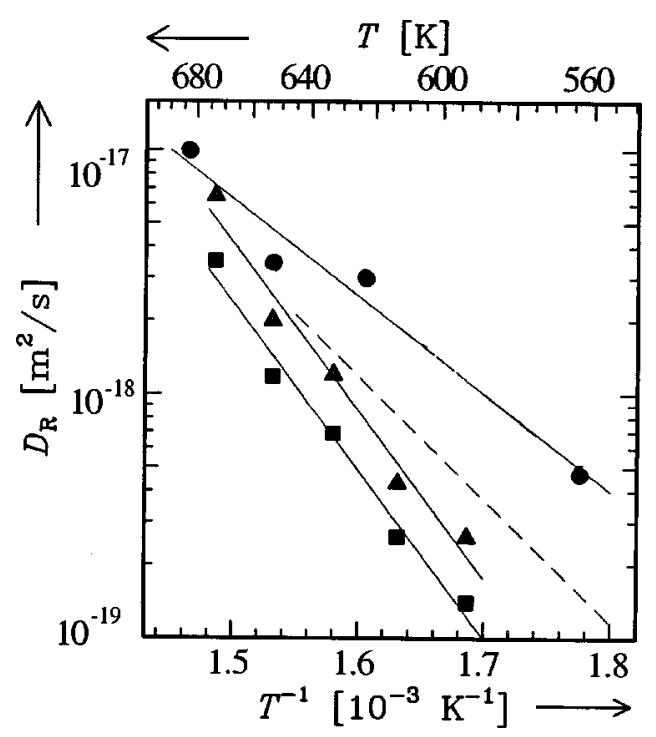

FIG. 25. Arrhenius plots of the self-diffusion coefficients of ${ }^{59} \mathrm{Fe}$ in relaxed amorphous $\mathrm{Fe}_{91} \mathrm{Zr}_{9}: \mathbf{\square}$, in the absence of irradiation; $\boldsymbol{\Delta}$, under irradiation with protons; dashed line, $\mathrm{He}^{+}$; - ${ }^{15} \mathrm{~N}^{+}$. After Schuler et al., 1997, 1999.

\section{Defects in metallic glasses and the nature of the $\delta$ phase}

In (nondecomposing) amorphous metallic alloys the number of potential types of defects is quite limited. Following Egami et al. (1980), defects in such materials may be defined as static local fluctuations of the mass density.

The simplest kinds of defects are dilution and compression centers of atomic size. These "point defects" represent elastic monopoles, which, in analogy to crystals, are referred to as "vacancies" and "(self-)interstitials," respectively. These terms are used for simplicity here, although defects in glasses are certainly different in structure and lifetime from their welldefined analogs in crystals. There is strong evidence from diffusion-under-irradiation experiments that hightransferred-energy irradiations produce distant vacancyinterstitial pairs (Frenkel pairs) whose partners separate, undergo free migration as individual entities, and thus give rise to radiation-enhanced diffusion (Sec. VIII.B.1).

When the energy transferred by irradiation drops to the Frenkel-pair production threshold, close vacancyinterstitial pairs are generated. In amorphous alloys, the minimum separation of the partners of such pairs may be smaller than in crystals-say, about one nearestneighbor-atom distance only. The reason for this is as follows. In a disordered structure, a vacant site produced by the displacement of an atom can be partly "closed" by a rearrangement of the surrounding atoms. In this way a barrier is produced that prevents the displaced atom from returning to "its" vacancy and thus keeps the close pair from complete spontaneous recombination. On the other hand, the elastic attraction between its dilution center and its compression center inhibits the pair from dissociating into a vacancy plus an interstitial. Such close pairs obviously constitute elastic dipoles, a second kind of elementary defect in amorphous alloys. They are immobile, since the atomic rearrangements required for their motion lead to their recombination. For the dipoles and small dipole clusters formed in relaxed amorphous $\mathrm{Co}_{58} \mathrm{Fe}_{5} \mathrm{Ni}_{10} \mathrm{Si}_{11} \mathrm{~B}_{16}$ by $0.4 \mathrm{MeV}-\mathrm{H}^{+}$irradiation, an average lifetime of $1500 \mathrm{~s}$ at $420^{\circ} \mathrm{C}$ has been estimated (Scharwaechter, 1993). These defects were identified with the radiation-induced, small spots of $\delta$ phase invoked to explain radiation-enhanced diffusion without a change in the diffusion enthalpy (Secs. VIII.A.2 and VIII.B.2).

A working hypothesis, which will turn out to be extraordinarily fruitful in elucidating the diffusion mechanisms in amorphous metallic alloys, concerns the equilibrium defects and their freezing in upon cooling. This hypothesis is that, under thermal equilibrium conditions, metallic solids contain vacancies and close Frenkel pairs, ${ }^{3}$ but no isolated self-interstitials (i.e., interstitials not involved in close Frenkel pairs). ${ }^{4}$ Rapid cooling leads to different nonequilibrium states for crystalline metals and amorphous metallic alloys. In crystals, only vacancies can be quenched in, whereas close Frenkel pairs cannot, since they recombine spontaneously. In metallic glasses, in addition to vacancies, close Frenkel pairs can be frozen in, since, as described above, a barrier impedes their recombination. It is proposed that the freezing in of extended clusters of close Frenkel pairs defines the caloric glass transition temperature $T_{g}$, which separates the deeply undercooled melt $\left(T_{c}>T\right.$ $\left.>T_{g}\right)$ from the glassy state $\left(T<T_{g}\right)$. These frozen-in close-Frenkel-pair agglomerates are believed to form a network, which is tentatively identified with the $\delta$-phase network introduced in Sec. VIII.A.2. At sufficiently high cooling rates, vacancies may also be frozen in below $T_{g}$.

It is well known that mild thermal annealing of asproduced metallic glasses leads to their relaxation into an amorphous metastable equilibrium state (Sec. III). This relaxation has been attributed to the annealing out of frozen-in vacancies, which is accompanied by a decrease in the diffusivity contribution mediated by these excess vacancies. In analogy to this "classical relaxation," in bulk metallic glasses long-time isothermal "super-relaxation" starting just below $T_{g}$ is expected to give rise to a dissolution of the close-Frenkel-pair network (which, via a decrease in $T_{g}$, leads to the deeply cooled melt state) and thus results in an annealing out of the diffusivity contributed by the $\delta$ phase. In fact, Zumkley, Naundorf, Macht, and Frohberg (2001) have recently demonstrated that by super-relaxation at $553 \mathrm{~K}$ the diffusivities of $\mathrm{B}$ and $\mathrm{Fe}$ in $\mathrm{Zr}_{46.8} \mathrm{Ti}_{8.2} \mathrm{Cu}_{7.5} \mathrm{Ni}_{10} \mathrm{Be}_{27.5}$ bulk glass at and below $553 \mathrm{~K}$ can be decreased significantly. The fact that the required super-relaxation times

\footnotetext{
${ }^{3}$ In thermal equilibrium, close Frenkel pairs represent local dynamic density fluctuations.

${ }^{4}$ Isolated self-interstitials do not occur in thermal equilibrium since, as a result of the large elastic strains they produce, their formation enthalpy is high. Whereas this is a matter of fact for metallic crystals, it is only tentatively transferable to metallic glasses.
} 
lay between $1.12 \times 10^{6}$ and $2.37 \times 10^{7} \mathrm{~s}$ is in accordance with the basic prerequisite for $\delta$-phase-mediated radiation-enhanced diffusion via mechanism (ii) that the lifetime of the frozen-in $\delta$ network considerably exceeds that of $\delta$ spots introduced by low-transferred-energy irradiation (Sec. VIII.A.2).

\section{Diffusion mechanisms in metallic glasses-a proposal stimulated by radiation-enhanced-diffusion results}

In super-relaxed metallic glasses, which contain neither quenched-in $\delta$ networks nor quenched-in vacancies, diffusion occurs exclusively via thermally activated displacement chains, i.e., a direct mechanism not involving point defects as diffusion vehicles (Sec. IX). This is true for all temperatures below $T_{c}$. In a given glass and at a given temperature there is a length distribution of the displacement chains. The diffusion of large atoms in a densely packed glass requires long chains (giving rise to large values of $H$ and $D_{0}$ ), whereas small atoms in an open glass can diffuse via short chains (resulting in small values of $H$ and $D_{0}$-compare Sec. V). The collective nature of displacement-chain diffusion smears out isotope effects, but leads to activation volumes $\Delta V$ that are unexpectedly large for a direct diffusion mechanism $(\Delta V / \Omega \approx 0.5 ; \Omega$ is the average atomic volume; see Secs. VI and IX).

In practice, metallic glasses can be produced by cooling from the melt at finite rates. The undercooled melts drop out of their metastable equilibrium when, at the (cooling-rate-dependent) glass transition temperature $T_{g}$, the $\delta$-phase network (consisting of close Frenkel pairs) is frozen in. At high enough cooling rates, vacancies are quenched in, too.

During diffusion annealing of unrelaxed metallic glasses, the quenched-in excess vacancies serve as diffusion vehicles and dominate diffusion until they have annealed out (Sec. III). Since hopping of atoms onto statically (i.e., not only during fluctuations) vacant neighboring sites is not a collective process, vacancymediated diffusion in unrelaxed metallic glasses shows a clear isotope effect (Sec. VII). ${ }^{5}$

In relaxed (but not super-relaxed) metallic glasses, the $\delta$ network provides fast diffusion paths for small diffusers. In this phase, the dilution centers of the close Frenkel pairs constitute sites which, assisted by thermal fluc-

\footnotetext{
${ }^{5}$ Thin films of amorphous $\mathrm{Co}_{51} \mathrm{Zr}_{49}$ and $\mathrm{Co}_{81} \mathrm{Zr}_{19}$ seem to be an exception. In these, Co diffusion does not show an isotope effect, either in the as-produced or in the relaxed state (Sec. VII). Within the framework of the ideas put forward in Sec. VIII.C, this may be interpreted as follows. In addition to the $\delta$-phase network, as-produced films contain $\delta$ spots similar to those introduced into nonfilm metallic glasses by lowtransferred-energy irradiation (Sec. VIII.B.2). In as-produced and in relaxed films as well, Co diffusion is controlled by the $\delta$ phase and therefore not sensitive to the isotope mass of the diffusing Co atoms (see next paragraph). In thin films the diffusivity decrease during relaxation reflects the annealing out of the $\delta$ spots rather than of excess vacancies.
}

tuations, may provide enough vacant space to enable neighboring small diffusers to jump onto them. By such an event a collective (e.g., ring-type) diffusion event comprising a small number of atoms is initiated. This mechanism combines in a unique way a small activation enthalpy and a negative activation entropy (i.e., a small $D_{0}$ value) with collective features that entail small or even vanishing isotope effects. Most interestingly, the mechanism is insensitive to hydrostatic pressure (Sec. VI) since, owing to the dipolar structure of the $\delta$ phase, the volume fraction of this phase cannot be changed by pressure.

Large diffusers do not fit in the diluted halves of closed Frenkel pairs and therefore cannot use the $\delta$ phase for fast diffusion. Hence, in relaxed metallic glasses, large atoms can only undergo displacementchain-type diffusion. This view is supported by the observation that the production of $\delta$ spots under proton irradiation (Sec. VIII.B.2) gives rise to an activationenthalpy-conserving diffusion enhancement of the small ${ }^{59} \mathrm{Fe}$ atoms in the open $\mathrm{Fe}_{91} \mathrm{Zr}_{9}$ (Fig. 25) but, under otherwise identical conditions, leaves unchanged the diffusivity of the large ${ }^{95} \mathrm{Zr}$ atoms in the densely packed $\mathrm{Fe}_{24} \mathrm{Zr}_{76}$ glass.

\section{Molecular-dynamics simulations of radiation-damage- induced diffusion}

Hamlescher (1994) (see also Frank et al., 1996; Scharwaechter et al., 1996b; Schuler et al., 1997, 1999) performed molecular-dynamics computer simulations of the production and annealing of radiation damage in relaxed amorphous $\mathrm{Fe}-\mathrm{Zr}$ alloys using cube-shaped "supercells" with 8000 atoms. By transferring kinetic energy to either an $\mathrm{Fe}$ or a $\mathrm{Zr}$ atom along a space diagonal of the supercell at $0 \mathrm{~K}$ damaging by irradiation was simulated. For instance, an energy transfer of $100 \mathrm{eV}$ to a $\mathrm{Zr}$ atom in $\mathrm{Fe}_{90} \mathrm{Zr}_{10}$ results in an agglomerate of close Frenkel pairs which undergoes only minor changes during subsequent annealing for $100 \mathrm{ps}$ at $600 \mathrm{~K}$. This confirms the great thermal stability of close Frenkel pairs in metallic glasses, particularly when these are present in the form of ( $\delta$-network-like) extended clusters. During the $600 \mathrm{~K}$ anneal, chainlike atomic displacements take place which are exclusively confined to the damaged region and resemble the thermally activated displacement chains found in Fe-Zr alloys by Hörner (1990, 1993; see also Frank et al., 1996; Scharwaechter et al., 1996b; Schuler et al., 1997, 1999) in simulations of diffusion (in the absence of irradiation) at higher temperatures (e.g., at $1655 \mathrm{~K}$ in $\mathrm{Fe}_{90} \mathrm{Zr}_{10}$ ). From this we conclude that, at least at intermediate temperatures (as represented by the simulated $600 \mathrm{~K}$ annealing), thermally activated displacement chains are located in defect regions of the amorphous structure, which is in accordance with previous findings in computer simulations, namely, onedimensional relaxations centered at structural irregularities (Schober, 1993; Schober et al., 1993; Schober and Oligschleger, 1996; Oligschleger and Schober, 1999) and mutual triggering of displacement chains (Teichler, 
2001). Simulated radiation-damage annealing at lower temperatures (e.g., in $\mathrm{Fe}_{90} \mathrm{Zr}_{10}$ at $200 \mathrm{~K}$ ) also gives rise to diffusive atomic displacements in the damaged region; however, under these conditions the displacements are not chainlike. This observation we interpret in terms of $\delta$-network-type facile diffusion that is undergone by small diffusers in relaxed, but not super-relaxed, bulk metallic glasses.

\section{COMPUTER SIMULATIONS}

\section{A. Introductory remarks}

On the atomic level, insight into details of the diffusion processes in metallic glasses and their melts has been gained from molecular-dynamics computer simulations. Actual simulations cover a time window of up to $0.7 \mu$ s (Teichler, 2001) and thereby are able to deal with diffusion coefficients down to $10^{-16} \mathrm{~m}^{2} / \mathrm{s}$. Under these conditions, the accessible simulation time is seven orders of magnitude larger than the scale of atomic vibrations and permits analyses of complex processes far beyond single-atom motions. Nevertheless, the limitation to some $10^{-16} \mathrm{~m}^{2} / \mathrm{s}$ restricts the analysis of diffusion to the range near and above the experimental glass transition temperature. This high-temperature regime, however, covers the important region where matter transport changes from viscous flow, characteristic of liquids, to the thermally activated diffusive transport relevant in the solid state.

In metallic glasses, this transition takes place in two steps. First, in the liquid, around a critical temperature $T_{c}$, there is a transition from the dynamics of a hightemperature state of homogeneous viscous flow to the dynamics of a low-temperature pseudoarrested state as predicted by the mode-coupling theory (Götze and Sjölander, 1992). Even well above $T_{c}$, the dynamics is heterogeneous in the form of coherent motions of chains or rings of atoms, while single-atom translational motion is suppressed by the rigidity of the cages of surrounding particles. Second, in the low-temperature state, when cooling below $T_{c}$, at the glass temperature $T_{g}$ the caloric glass transition occurs, as indicated by a jump in the specific heat or a change in the volume expansion coefficient. $T_{g}$ shows the well-known cooling-rate dependence, which reflects the nonequilibrium, kinetic effects determining the entrance into the glassy state.

Molecular-dynamics simulation of atomic motion in metallic glasses and related model systems has been carried out for a long time (e.g., Kimura and Yonezawa, 1983; Miyagawa et al., 1988; Brandt, 1989; Lewis, 1991). Recent studies even consider complicated systems like a ternary, bulk-glass-forming alloy (Guerdane, 2000). The molecular-dynamics results on matter transport in metallic melts and glasses rely on isothermal simulations for a finite piece of material, containing $N$ atoms, which is periodically repeated in space in order to model bulk samples. In the treatments, $N$ varies from some hundred atoms to several tens of thousands, depending upon whether long-time or large-system effects are investigated. In the simulations, the Newtonian equations of

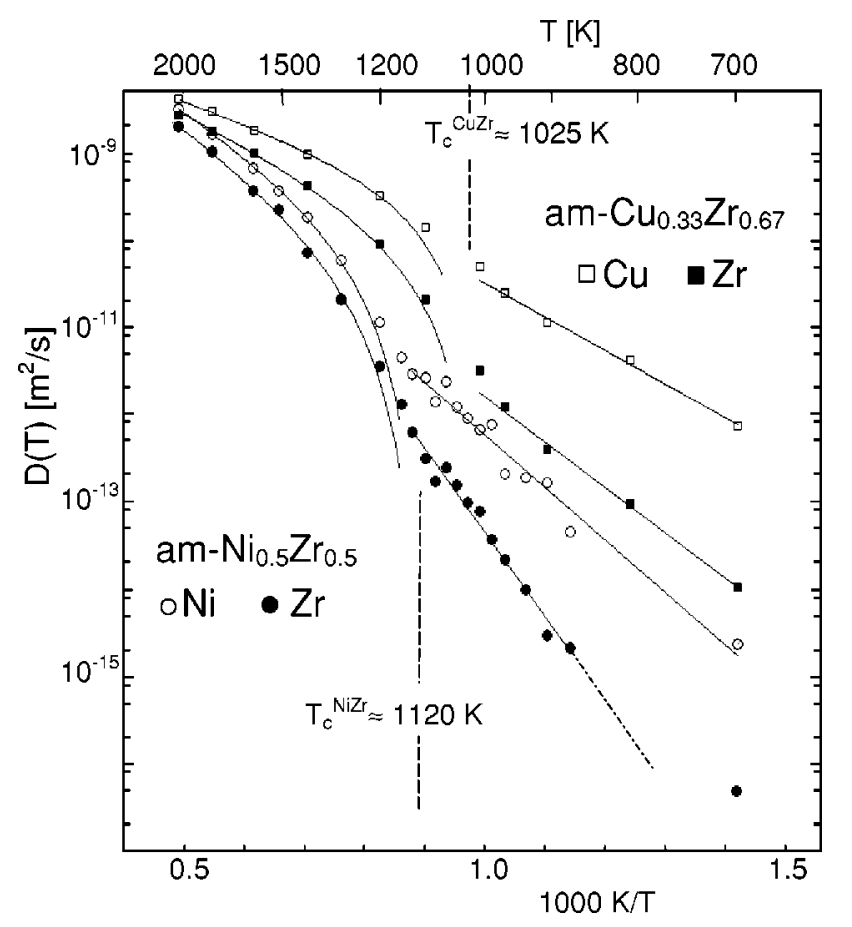

FIG. 26. Arrhenius plot of molecular-dynamics-simulated diffusion coefficients for molten and glassy $\mathrm{Ni}_{0.5} \mathrm{Zr}_{0.5}$ (Teichler, 1997, 2001) and $\mathrm{Cu}_{0.33} \mathrm{Zr}_{0.67}$ (Kluge, 2001).

motion of the atoms are numerically integrated, either in the isobaric $(N, T, p)$ mode under constant pressure $p$ or in the isochoric $(N, T, V)$ mode under constant volume $V$. Temperature is usually measured by the mean kinetic energy of the atoms. (Details may be found in textbooks, e.g., Allen and Tildesley, 1987.) An essential point in the simulations is the modeling of the interatomic couplings. Here the approaches range from schematic soft sphere and Lennard-Jones models over heuristic pair-potential descriptions (e.g., van Ee et al., 1998) to microscopically well-founded treatments of atomic metallic interactions, like the embedded-atom method (Baskes and Johnson, 1994) with its empirical input parameters or the Hausleitner-Hafner (1992) approach based on theory. In the more recent simulations, due to increased computer capacity, much better relaxed structures could be generated than in earlier studies, which means more reliable microscopic models of the relaxed amorphous state could be analyzed.

In the following, we discuss the overall temperature dependence of matter transport in undercooled metallic melts and glasses, as obtained from molecular-dynamics simulations, the modes of atomic motion in these glasses and melts, as revealed by the numerical studies, the isotope effect of diffusion, the activation volume of diffusion, dynamical heterogeneity in the melts, and the consequences of reduced dimensionality. Moleculardynamics simulations of diffusion in glasses under particle irradiation are reported in Sec. VIII.

\section{B. Molecular-dynamics results concerning diffusion coefficients in melts and glasses}

As a typical example, Fig. 26 shows the diffusion coefficients of $\mathrm{Ni}$ and $\mathrm{Zr}$ in $\mathrm{Ni}_{0.5} \mathrm{Zr}_{0.5}$ (Teichler, 1997, 2001) 
and of $\mathrm{Cu}$ and $\mathrm{Zr}$ in $\mathrm{Cu}_{0.33} \mathrm{Zr}_{0.67}$ (Kluge and Schober, 2001), calculated from the long-time evolution of the mean square displacements,

$$
\begin{aligned}
& D^{\ell}(T)=\lim _{t \rightarrow \infty} 1 / 6 \partial_{t}\left\langle u^{2}(t)\right\rangle^{\ell}, \\
& \left\langle u^{2}(t)\right\rangle^{\ell}=\left\langle\left|\boldsymbol{R}^{n}\left(t+t_{0}\right)-\boldsymbol{R}^{n}\left(t_{0}\right)\right|^{2}\right\rangle_{n \in \ell},
\end{aligned}
$$

where \langle\rangle$_{n \in \ell}$ denotes an averaging over the positions $\boldsymbol{R}^{n}$ of all atoms $n$ of the species $\ell$ and over the starting times $t_{0}$.

Three temperature regimes can be distinguished. In both materials in the hot melt $(T>1500 \mathrm{~K})$ the diffusion coefficients of both components are similar. The small differences between the $D$ of the two components were expected because of the atomic radii and masses. Upon cooling, diffusion drops faster than described by the Arrhenius law [Eq. (4.1)] and is often described by a Vogel-Fulcher law. This slowing down is in good agreement with the prediction of the mode-coupling theory (Götze and Sjölander, 1992):

$$
D^{\ell}(T) \propto\left|T-T_{c}\right|^{\gamma} .
$$

The molecular-dynamics data can be fitted with modecoupling critical temperatures $T_{c}=1120$ and $1025 \mathrm{~K}$ for the $\mathrm{Ni}_{0.5} \mathrm{Zr}_{0.5}$ and $\mathrm{Cu}_{0.33} \mathrm{Zr}_{0.67}$ melts, respectively. In the case of $\mathrm{Ni}_{0.5} \mathrm{Zr}_{0.5}, T_{c}$ was determined from the decay of the density-density correlation function (Teichler, 1996) rather than from the diffusion data. These temperatures are a few percent higher than the corresponding glass transition temperatures obtained under the computationally accessible cooling rates, $T_{g}=1050 \mathrm{~K}\left(\mathrm{Ni}_{0.5} \mathrm{Zr}_{0.5}\right.$ : Teichler, 1996) and $970 \mathrm{~K}\left(\mathrm{Cu}_{0.33} \mathrm{Zr}_{0.67}\right.$ : Kluge and Schober, 2001). Considering the high effective quench rates, of the order of $10^{9} \mathrm{~K} / \mathrm{s}$ in the simulations, these values are reasonable (Teichler, 1999). The $\gamma$ values are different for the two components: NiZr, $\gamma_{\mathrm{Ni}}=2.65, \gamma_{\mathrm{Zr}}$ $=3.0 ; \mathrm{CuZr}, \gamma_{\mathrm{Cu}}=1.49, \gamma_{\mathrm{Zr}}=2.13$.

Below $T_{c}$ the diffusion follows the classical Arrhenius law (4.1) with $D_{0}=5 \times 10^{-7}$ and $1 \times 10^{-4} \mathrm{~m}^{2} / \mathrm{s}$ and $H$ $=1.2$ and $2.0 \mathrm{eV}$ in $\mathrm{Ni}_{0.5} \mathrm{Zr}_{0.5}$ for $\mathrm{Ni}$ and $\mathrm{Zr}$, respectively (Teichler, 1997), and $D_{0}=1.7 \times 10^{-7}$ and 11.3 $\times 10^{-7} \mathrm{~m}^{2} / \mathrm{s}$ and $H=0.75$ and $0.99 \mathrm{eV}$ in $\mathrm{Cu}_{0.33} \mathrm{Zr}_{0.67}$ for $\mathrm{Cu}$ and $\mathrm{Zr}$, respectively (Kluge and Schober, 2001). The values for $\mathrm{Ni}$ diffusion in $\mathrm{Ni}_{0.5} \mathrm{Zr}_{0.5}$ are in good agreement with the experimental ones $\left(D_{0}=1.7 \times 10^{-7} \mathrm{~m}^{2} / \mathrm{s}\right.$, $H=1.3 \mathrm{eV}$; Averback, 1991).

This behavior is general and is observed in different metallic and nonmetallic glasses and glass-forming liquids. It does not depend on details of the description of the interatomic interaction. Two completely different approaches were used to model the two substances of Fig. 26, viz., the Hausleitner-Hafner (1992) approach for $\mathrm{Ni}-\mathrm{Zr}$ and an embedded-atom method (Baskes and Johnson, 1994) for $\mathrm{Cu}-\mathrm{Zr}$. There are, however, quite strong quantitative dependencies on the atomic species involved and on their relative concentrations. In Fig. 26, for instance, it is apparent that upon cooling to $T_{c}$ the splitting between the $\mathrm{Cu}$ and $\mathrm{Zr}$ diffusivities in $\mathrm{Cu}_{0.33} \mathrm{Zr}_{0.67}$ increases more strongly than the corre-

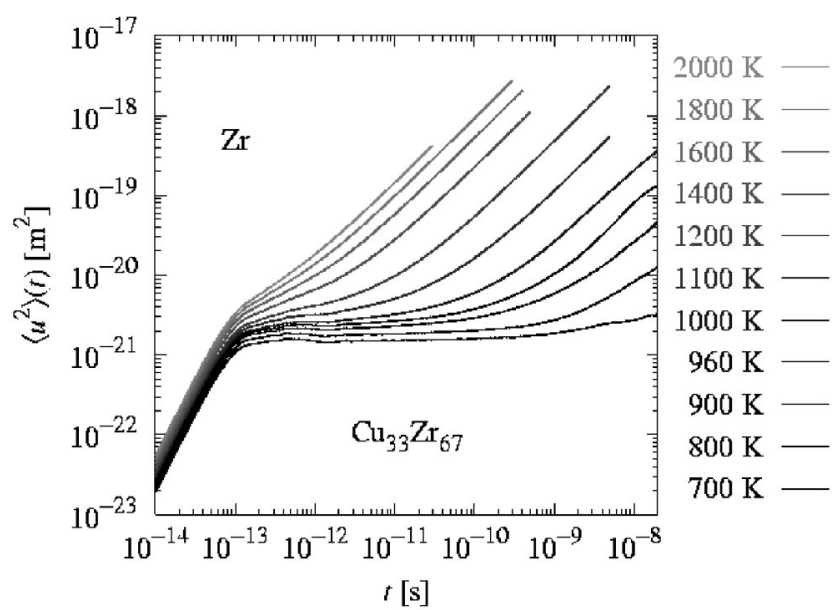

FIG. 27. Average mean square displacement of $\mathrm{Zr}$ in $\mathrm{Cu}_{0.33} \mathrm{Zr}_{0.67}$ as a function of time on a double logarithmic scale. Temperatures vary from $2000 \mathrm{~K}$ (top) to $700 \mathrm{~K}$ (bottom). After Kluge, 2001.

sponding splitting in $\mathrm{Ni}_{0.5} \mathrm{Zr}_{0.5}$. This is plausible since the structure is much less influenced by $\mathrm{Cu}$ in the first case than by $\mathrm{Ni}$ in the second case. A splitting similar to the behavior in $\mathrm{Cu}_{0.33} \mathrm{Zr}_{0.67}$ is found in $\mathrm{Ni}_{0.8} \mathrm{Zr}_{0.2}$ (Teichler, 1997). In a simulation of the related $\mathrm{Co}_{1-x} \mathrm{Zr}_{x}$ system, Rößler and Teichler (2000) have shown that, with varying concentration, the temperature $T_{c}$ varies between 650 and $1150 \mathrm{~K}$, grossly scaling with the liquidus temperature in this system. Lines of constant averaged diffusivity in the undercooled melt scale with this temperature, too. The concentration and temperature dependencies of the diffusivities of the single components are more intricate.

The different temperature regimes are reflected in the time evolution of the atomic mean square displacements, as shown in Fig. 27 for $\mathrm{Zr}$ in $\mathrm{Cu}_{0.33} \mathrm{Zr}_{0.67}$ (Kluge, 2001) on a logarithmic time scale. For times below $0.1 \mathrm{ps}$, the mean square displacement increases for all temperatures proportional to $t^{2}$, as expected for both ballistic motion and vibrations. The absolute value increases only slightly with temperature. For long times the mean square displacement increases linearly with time, which is the signature of long-range diffusion. This leads to a complete decay of correlation, frequently called $\alpha$ relaxation. In the hot melt this relaxation is on time scales of picoseconds. Upon cooling, diffusion slows down, which shows up as a shift to the right, and a shoulder appears. This indicates a faster process (fast $\beta$ relaxation), which does not markedly contribute to longrange diffusion. Upon cooling into the amorphous state, the shoulder turns into a plateau. The $\beta$ relaxations are local processes. These time and temperature regimes are observed in many different quantities. See, for example, Kob and Andersen (1995).

In contrast to most experiments and statistical theories, one can follow the motion of single atoms in molecular-dynamics simulations. Therefore simulations are ideally suited for answering questions as to the atomic origin of the observed effects. At low tempera- 
tures, in the amorphous state isolated jump processes can be identified. With increasing temperature, the resolution of single processes is no longer possible and, eventually, these merge into a flow motion. In the following, we shall review results concerned with dynamics on the atomic scale. We shall start with the more straightforward case of an amorphous solid.

\section{Motion in an amorphous solid}

At the lowest temperatures, the dynamics in amorphous materials are characterized by three types of local excitations (tunneling systems, excess vibrations, and local relaxations), which coexist and interact with sound waves. A common description of these excitations is provided by the soft-potential model. Fitting the model to the available experimental data of three different glasses, Buchenau et al. (1991) found that all three excitations involve collective motions of 20 or more atoms. From a molecular-dynamics simulation of a soft-sphere glass, Laird and Schober (1991) found that the excess in the vibrational spectrum at the lowest frequencies is indeed due to quasilocalized (resonant) vibrations of groups of ten and more atoms. (At higher frequencies the interaction between these modes and with the sound waves becomes important, which leads to the "boson peak.") Similar results were subsequently obtained for numerous other materials.

Localized soft vibrations provide a mechanism for reaching large atomic amplitudes, a prerequisite for hopping over barriers at low temperatures. Indeed it was found that the displacements in an atomic jump are closely related to resonant vibrations involving the jumping atoms (Schober, 1993; Oligschleger and Schober, 1999). Figure 28 shows an example of the initial and final positions of the most active atoms in such a jump. The total jump length is about 1.5 nearestneighbor distances, but a single atom moves only a fraction of this distance. Typical is the chain-type process, which is the easiest way in which atoms can propagate collectively. It involves only slight changes of the density at the ends of the chain, which in part are accommodated by long-range elastic displacements. The chain structure of the jumps causes their structure factor to closely reflect the static structure factor of the glass (Gaukel et al., 1999).

As the temperature is increased the total jump lengths and the jump lengths of the individual atoms as well as the number of atoms involved in a jump increase (Schober et al., 1997; Oligschleger and Schober, 1999). Eventually some atoms jump over nearest-neighbor distances. This is observed particularly for smaller minority species in binary and multicomponent systems. The number of collectively jumping atoms may depend on the chemical composition of the glass, as demonstrated by comparing $\mathrm{Ni}_{0.8} \mathrm{Zr}_{0.2}$ and $\mathrm{Ni}_{0.5} \mathrm{Zr}_{0.5}$ (Teichler, 1997).

At low temperatures, computer power does not allow discrimination between local relaxations which are even-

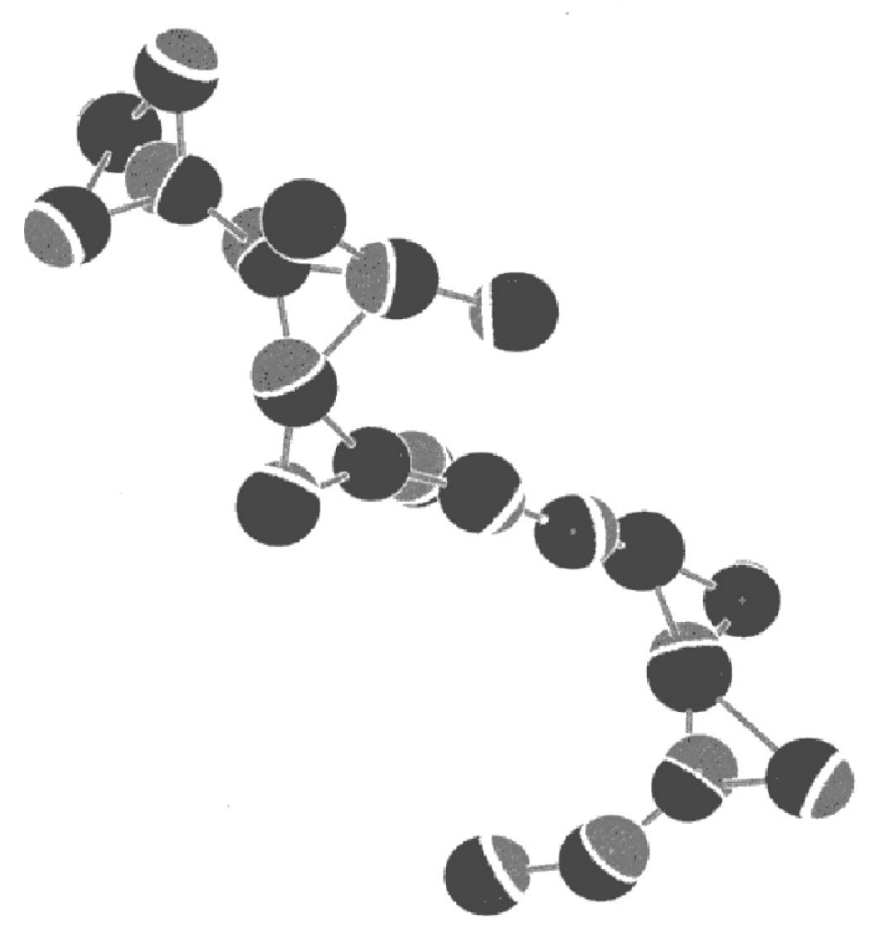

FIG. 28. Group of atoms moving collectively in a single-jump process in a soft-sphere glass at $T=0.125 T_{g}$. Shown are all atoms with amplitudes of more than $40 \%$ of the maximal one (dark circles, initial position; light circles, final position). After Schober et al., 1993.

tually reversed and those leading to long-range diffusion. However, there is no indication of two different mechanisms.

An important observation is the correlation between jumps (Teichler, 1997, 2001; Oligschleger and Schober, 1999). First, subsequent jump processes in the same part of the sample largely involve the same atoms. Second, jumps trigger each other. This can be seen in Fig. 29, which shows the mean square displacement, not averaged over the starting times, in a sample of $\mathrm{Ni}_{0.5} \mathrm{Zr}_{0.5}$ at

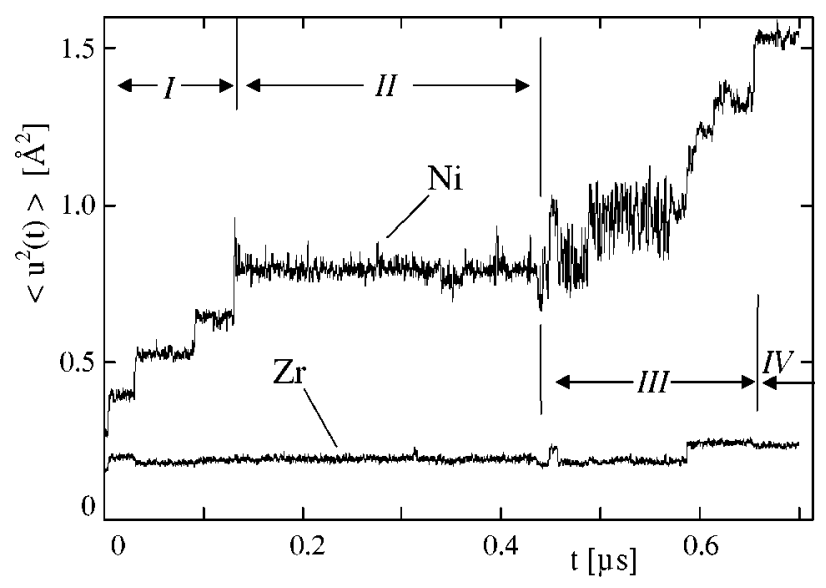

FIG. 29. Mean square displacements of $\mathrm{Ni}$ and $\mathrm{Zr}$ in ultralong molecular-dynamics-simulated $\mathrm{Ni}_{0.5} \mathrm{Zr}_{0.5}$ at $700 \mathrm{~K}$, showing bursts of dynamical activity after a period of $300 \mathrm{~ns}$ of arrest. After Teichler, 2001. 
$700 \mathrm{~K}$, i.e., well below the glass transition. As expected from Fig. 26, the activity at this temperature is mainly concentrated on $\mathrm{Ni}$ atoms. Four time regimes can be distinguished: the region of initial increase of the mean square displacement by isolated irreversible chain transitions, which is followed by a period of relative calm, then a burst of activity with a number of successive chain transitions, and then again a period of rest. Interdispersed are single spikes indicating jumps that are rapidly reversed. The curves for $\mathrm{Ni}$ and $\mathrm{Zr}$ are correlated; this shows that $\mathrm{Zr}$ participates weakly in the motion of the $\mathrm{Ni}$ atoms. These results were taken as indications (Teichler, 2001) that in the well-relaxed glassy state at experimentally relevant temperatures diffusion takes place by means of such bursts of mutually triggering chain transitions rather than by statistically independent events.

\section{Motion in a liquid}

The situation for molecular-dynamics simulations in liquids is opposite to that in the solid state. On the one hand, one can no longer easily study elementary processes (jumps) directly. On the other hand, statistical quantities such as diffusion coefficients can readily be computed, and aging is no problem. To gain some insight, the velocity correlation function has often been studied in liquids, e.g., by Balucani and Zoppi (1994). Near $T_{c}$ it develops long oscillating tails, which make an exact evaluation difficult.

The change of dynamics upon cooling towards the glass transition can clearly be seen in the self-part of the van Hove function $G^{s}(r, t)$, which is related to the probability that an atom has moved by a distance $r$ during a time $t$ :

$$
P(r, t)=4 \pi r^{2} G^{s}(r, t)=\left\langle\delta\left(r-\left|\boldsymbol{R}^{n}(t)-\boldsymbol{R}^{n}(0)\right|\right)\right\rangle .
$$

At high temperatures $P(r, t)$ is, apart from the geometrical factor $4 \pi r^{2}$, nearly perfectly Gaussian and broadens $\propto \sqrt{t}$. Upon cooling towards $T_{c}$ and below, the selfcorrelation function becomes markedly non-Gaussian, and a tail to larger distances grows with time. Finally, on approaching $T_{c}$, additional structure evolves, particularly for the more mobile components (Roux et al., 1989; Wahnström, 1991; Kob and Andersen, 1995; Teichler, 1997; Gaukel and Schober, 1998). As an example, Fig. 30 shows the distribution of the atomic displacements in $\mathrm{Zr}_{0.67} \mathrm{Cu}_{0.33}$ at $1000 \mathrm{~K}$ after $210 \mathrm{ps}$, i.e., during the early time of the so-called $\alpha$ relaxation. The second peak grows at a fixed, time-independent position roughly equal to the mean nearest-neighbor distance.

From this structure of $P(r, t)$, one can conclude that there are preferred positions on the relevant time scales. This, however, does not necessarily mean that these positions are reached in a single jump, as frequently assumed. From an analysis of the evolution with time it has been concluded that the evolution of this structure in $P(r, t)$ is accompanied by a strong increase of backcorrelation (Gaukel and Schober, 1998).

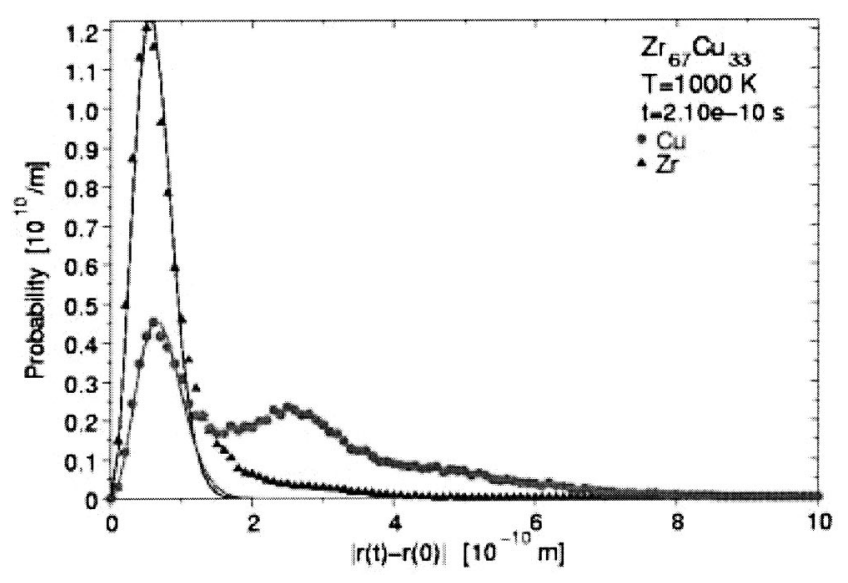

FIG. 30. Distribution of the atomic displacements $P(r, t)$ in $\mathrm{Cu}_{0.33} \mathrm{Zr}_{0.67}$ after 210 ps at $1000 \mathrm{~K}$ : symbols, moleculardynamics simulation; lines, Gaussian fit to the short-range part. After Gaukel et al., 1999.

Either tracking single jumps or taking snapshots of the system at different times, one finds that groups of atoms, mainly forming chains, move collectively in both model systems (Miyagawa et al., 1988; Wahnström, 1991; Donati et al., 1998; Schrøder and Dyre, 1998) and in NiZr (Teichler, 1997) and CuZr (Schober et al., 1997). Upon cooling, the chain lengths grow (Donati et al., 1998). Depending on the method adopted by the authors, sometimes only chains of atoms moving to a nearest-neighbor position are taken into account. These constitute replacement chains, which may in part be closed loops. The chains will predominantly comprise atoms of the more mobile components for which one finds the more pronounced secondary peaks in $P(r, t)$. As for the diffusion coefficients, one expects a complicated dependence of $P$ on the relative concentrations. A small component of low concentration will need less collectivity to move than a large majority component. The picture of moving chains implies a small isotope effect and is closely connected to the dynamic heterogeneity at intermediate times.

\section{E. Isotope effect}

Direct calculations of the isotope-effect parameter, $E$ [Eq. (7.1)], by molecular-dynamics simulations require good statistics. To avoid clustering effects or changes of $T_{c}$ with mass, high concentrations of heavy or light isotopes and of large mass differences should be avoided. Therefore no direct calculation of the isotope effect in the glassy state is available so far. However, we have seen above that motion in the glassy state takes place by collective jumps. This implies an isotope effect of the order $1 / N_{a}$, where $N_{a}$ is the number of atoms participating in the jump (Fig. 28). From the high collectivity observed in a soft-sphere system, in $a$-Se, and in $\mathrm{Cu}_{0.33} \mathrm{Zr}_{0.67}$, one estimates $E<0.1$.

Recently the isotope effect in monatomic and binary Lennard-Jones systems was calculated as a function of 
temperature and density (Kluge and Schober, 2000; Schober, 2001). Cooling from high temperatures to $T_{c}$, the isotope effect drops from about 0.3 to 0.05 . In a monatomic system, this drop can be fully accounted for by the increase in density. In a binary system, a different scaling for the two components is found. These results are in full agreement with the experimental findings discussed in Sec. VII.

\section{F. Activation volume}

A particular signature of a diffusion process is its activation volume, i.e., the pressure dependence of the activation enthalpy. For a number of glasses, experimental values of this quantity are available (Sec. VI). Molecular-dynamics-simulation data on the activation volume are scarce. One estimate has been given recently (Teichler, 2001) for $\mathrm{Ni}-\mathrm{Zr}$ at about $700 \mathrm{~K}$ within the framework of the above-sketched microscopic picture of matter transport in metallic glasses. For this temperature it was shown that irreversible atomic displacements in the Ni subsystem are due to the motion of chains of atoms over barriers of 1-1.6 eV in the energy landscape of the system. This fits reasonably well to the simulated activation enthalpy for $\mathrm{Ni}$ diffusion of $1.2 \mathrm{eV}$ (experimental value $1.3 \mathrm{eV}$ ). For an arbitrarily selected barrier of $1.2 \mathrm{eV}$, it was further deduced that the corresponding barrier height $\Delta U$ increases under hydrostatic pressure $p$ according to $\Delta U(p)=\Delta U(0)+p V_{a c t}$ with an activation volume $V_{a c t}=7 \times 10^{-30} \mathrm{~m}^{3}=0.43 V_{a c t}$, where $V_{a c t}$ is the experimental, averaged atomic volume of the system (Teichler, 2001). For Co (as a substitute for Ni tracers) in $\mathrm{Ni}_{x} \mathrm{Zr}_{1-x}$ experiments yielded activation volumes of $V_{a c t}(x=0.52)=2 \times 10^{-29} \mathrm{~m}^{3} \quad$ (Höfler et al., 1992), $V_{a c t}(x=0.42)=8.9 \times 10^{-30} \mathrm{~m}^{3}$, and $V_{a c t}(x=0.62)=7.9$ $\times 10^{-30} \mathrm{~m}^{3}$ (Sec. VI). Hence the experimentally observed values for the activation volume are in agreement with the microscopic picture that matter transport in metallic glasses occurs via a thermally activated, coherent motion of chains of atoms, where a significant part of the observed $\Delta \Omega$ reflects the migrational part of the activation volume of the chains.

\section{G. Heterogeneity}

As mentioned before, diffusion in both the glassy state and the undercooled liquid is heterogeneous on not-too-long time scales. This can be seen either directly by checking which atoms have moved over a given distance in some time interval or by checking the deviation of $P(r, t)$ [Eq. (9.4)] from a Gaussian distribution. An often used quantitative measure of this deviation is the non-Gaussianity parameter (Rahman, 1964),

$$
\alpha_{2}=3 / 5\left\langle u^{4}(t)\right\rangle /\left\langle u^{2}(t)\right\rangle^{2}-1,
$$

where $\left\langle u^{2}(t)\right\rangle$ and $\left\langle u^{4}(t)\right\rangle$ are the mean quadratic and quartic displacements [Eq. (9.1)]. A Gaussian distribution of atomic displacements, as found for a random walk or for homogeneous harmonic vibrations, gives $\alpha_{2}=0$. The limiting value for times $t=0$ and $t=\infty$ is $\alpha_{2}$
$=0$. For $t=\infty$, this limit reflects the ergodicity of the system for long times. Starting from $t=0, \alpha_{2}(t)$ rises monotonically to a maximum and then drops monotonically. The maximum value is about 0.2 in the hot liquid and increases strongly in the undercooled liquid, where it reaches values close to 3.0. Cooling to temperatures below the glass transition raises the maximum nonGaussianity dramatically. The time position of the maximum is at high temperatures in the ps range and correlates in the supercooled liquid with the onset of the $\alpha$ relaxation, which is attributed to long-range motion.

We have seen that, on an atomic level, the dynamics in glasses and in undercooled liquids are very similar. This is also seen in $\alpha_{2}(t)$, which increases in both states proportional to $\sqrt{t}$. At about $0.7 T_{g}$, this behavior stretches over several nanoseconds. It can be shown that this dependence is a signature of collectivity and dynamic heterogeneity (Caprion et al., 2000). For long times when homogeneity is gradually restored one finds $\alpha_{2}(t) \propto 1 / t$. In the rigid glassy state the decay of $\alpha_{2}(t)$ shifts to macroscopic times. This can be envisaged from the ultralong process shown in Fig. 29. During this process, in the whole time window from 0.135 to $0.700 \mu \mathrm{s} \alpha_{2}(t)$ remains close to 18.7 , except for fluctuations.

\section{H. Reduced dimensionality}

So far we have considered simulations of atomic dynamics in bulk melts and glasses. Recent years have seen a growing interest in the dynamics under reduced dimensionality, e.g., in thin films with free surfaces or rigid boundaries, or in cylindrical tubes. These computer studies and their experimental counterparts (see, for example, Park and McKenna, 2000) aim at investigating whether there is a critical length-scale dependence associated with the dynamics during the glass transition. In an early molecular-dynamics approach, Fehr and Löwen (1995) studied a liquid, monatomic Lennard-Jones system confined between parallel plates. Besides a shift of the glass transition to higher temperatures, they reported the qualitative observation that "the layers near the wall exhibit a much slower diffusion."

More quantitative results were obtained by moleculardynamics investigations of thin films with free surfaces for the above-described Ni-Zr model (Böddeker, 1999; Böddeker and Teichler, 1999) and films bounded on one side by rigid walls (Böddeker, 1999). From the simulations, depth $(z)$-dependent chemically averaged diffusion coefficients, $D(z)=c_{\mathrm{Zr}} D_{\mathrm{Zr}}+c_{\mathrm{Ni}} D_{\mathrm{Ni}}$, were determined, as shown in Fig. 31 for a $\mathrm{Ni}_{0.5} \mathrm{Zr}_{0.5}$ film of $4.2 \mathrm{~nm}$ thickness with two free surfaces in the temperature regime around $T_{c}$ and $T_{g}$. In the interior, $D(z)$ takes the bulk value, $D(\infty)$ (Fig. 26), whereas the surface value $D(0)$ is enhanced by about two orders of magnitude within a transition layer of about $1 \mathrm{~nm}$. A detailed analysis indicates that on this length scale neither significant density fluctuations nor chemical decompositions take place.

A similar reduction of diffusion is seen at rigid boundaries (Böddeker, 1999). The effects of solid boundaries on atomic motion in undercooled melts can also be in- 


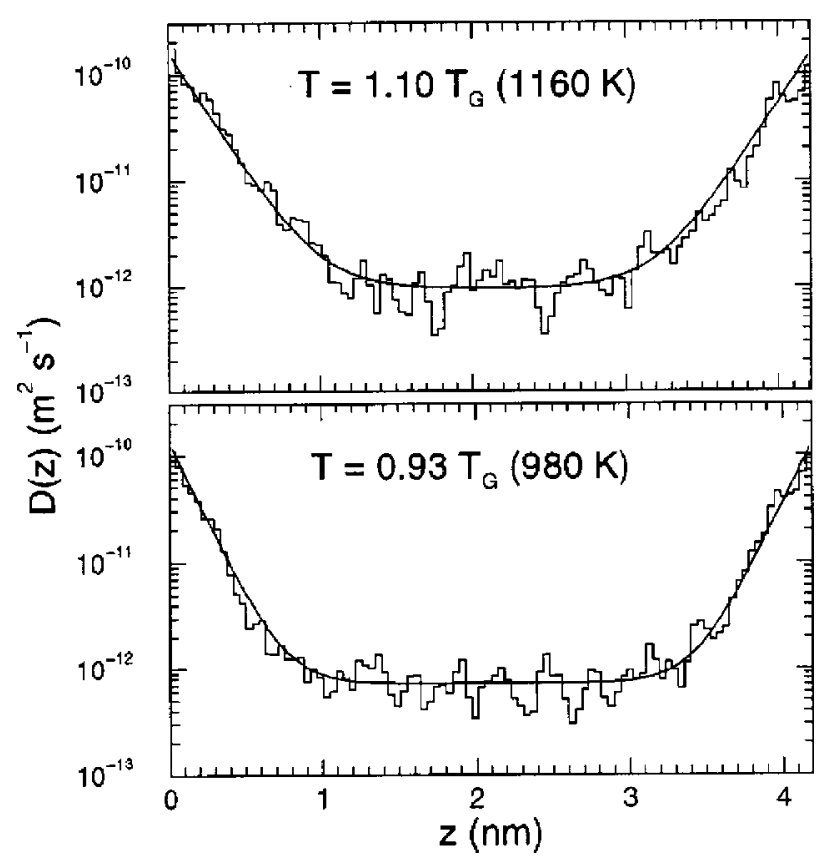

FIG. 31. Molecular-dynamics-simulated profiles of effective diffusion coefficients across a free-standing $\mathrm{Ni}_{0.5} \mathrm{Zr}_{0.5}$ film of 4.2 $\mathrm{nm}$ thickness for temperatures around the dynamic and caloric glass transition regime. After Böddeker and Teichler, 1999.

ferred from a molecular-dynamics study of Scheidler, Kob, and Binder (2000) of the relaxation dynamics of a binary Lennard-Jones liquid that is confined to a narrow cylindrical tube. Measuring the simulated dynamics of the particles as a function of their distance from the wall, it is found that the relaxation times of the density correlation functions increase strongly for short distances from the wall.

\section{SUMMARY AND CONCLUDING REMARKS}

Recent progress in the area of diffusion in conventional and bulk metallic glasses and their supercooled melts has been reviewed in the present paper. The major difference between conventional and bulk metallic glasses lies in the fact that the latter exhibit a glass transition at temperatures below the onset of crystallization. Therefore bulk metallic glasses can be transformed by heating into their supercooled liquid state. By contrast, conventional metallic glasses crystallize before they reach the glass transition. Particular attention has been devoted to the understanding of the atomic mechanisms of diffusion in metallic glasses.

Most of the reliable diffusion studies on metallic glasses were performed by the radiotracer technique (Sec. II). Usually, sputter profiling, which has a high depth resolution, was used because of the low diffusivities in metallic glasses. An important advantage of the radiotracer technique is that it can study self-diffusionthe most basic diffusion process. Methods that can compete with the radiotracer technique are secondary ion mass spectroscopy (SIMS) and Auger electron spectroscopy (AES) in combination with sputter profiling. Indi- rect estimates of self-diffusion coefficients, e.g., from crystal growth studies, should be considered with caution. The mechanism and kinetics of such a process are usually established from known diffusion data rather than vice versa.

Structural relaxation of metallic glasses leads from a less stable to a more stable-but still metastableamorphous or supercooled liquid state (Sec. III). It is accompanied by changes in many physical properties. In particular, the diffusivities decrease during thermal annealing. Structural relaxation is caused by the annealing out of quenched-in excess volume. In conventional metallic glasses, the excess volume has been found to exhibit vacancylike features ("quasivacancies"). Long-time annealing of bulk metallic glasses leads to the supercooled liquid state. Conventional metallic glasses cannot undergo this type of relaxation ("super-relaxation" or "long-time relaxation") since this is prevented by the onset of crystallization.

As in crystalline solids, diffusion in metallic glasses is thermally activated (Sec. IV). In relaxed conventional metallic glasses the diffusion coefficients show Arrhenius-type temperature dependencies over the entire temperature ranges covered by the experiments. Comparing the logarithm of the preexponential factors and the activation enthalpies for various diffusers in relaxed metallic glasses, a linear relationship-like that for solute diffusion in crystalline metals and/or alloys-is obtained. However, for metallic glasses the variation of the preexponential factors is orders of magnitude larger than for crystalline metals. In the latter, both selfdiffusion and diffusion of substitutional solutes occur by a vacancy mechanism; only solutes that are considerably undersized diffuse via an interstitial mechanism. This distinct difference between diffusion in crystalline metals and metallic glasses makes diffusion by means of single-atom jumps in metallic glasses unlikely.

For several diffusers in bulk metallic glasses a break in the Arrhenius plots of the diffusivities has been observed. The preexponential factors and activation enthalpies are higher above than below this break. It has been demonstrated that the break occurs at the transition from the supercooled liquid to the glassy state. The break in the Arrhenius plot separating the supercooled liquid and the glassy state can be shifted to lower temperatures by long-time annealing. In the supercooled state the slope of the Arrhenius plot has to be regarded as an effective quantity. It does not reflect an activation enthalpy due to the structural changes occurring above the caloric glass transition temperature, Moreover, molecular-dynamics simulations (Sec. IX) show that the linear Arrhenius behavior observed in diffusion studies is only due to the limited temperature range of the measurements. A downward curvature is expected at higher temperatures.

The atomic-size dependence of the activation enthalpy of self- and solute diffusion in metallic glasses is distinctly different from that of solute diffusion in crystalline solvents (e.g., noble metals), in which electrostatic interaction between solute and vacancy-type de- 
fects has a prominent influence on the transport mechanism (Sec. V). Rather, diffusion in metallic glasses is similar to solute diffusion in crystalline solvents such as lead, where the elastic interaction dominates solute diffusion. Molecular-dynamics simulations of diffusion processes strongly support the view that an important diffusion mechanism in metallic glasses is the migration of atoms by thermally activated displacement chains or displacement rings. This is corroborated by the dependence of the activation enthalpies on the size of the diffusing atoms.

Studies of the pressure dependence of diffusion and the activation volumes deduced from these have been critical in elucidating diffusion mechanisms in crystalline solids. For vacancy-mediated diffusion in crystalline metals, the activation volume consists of the formation volume and the migration volume of the vacancy. The formation volume lies typically between 0.5 and 1 atomic volumes, whereas the migration volume plays only a minor role, at least for pure metals. For direct interstitial diffusion of small solutes, no defect formation volume is involved, i.e., the activation volume equals the migration volume of the interstitial.

Measurements of the pressure dependence showed that diffusion in relaxed metallic glasses (Sec. VI) can be grouped into two categories:

Systems with almost-vanishing pressure dependence: Activation volumes close to zero are observed for metallic glasses which mainly contain late transition elements and for tracers of the size of the majority component. These very small activation volumes allow diffusion analogous to the vacancy mechanism to be ruled out and have been taken as evidence for a direct, collective diffusion mechanism, which does not involve the formation of thermal defects.

(ii) Systems with nonvanishing pressure dependence: Activation volumes comparable to those of vacancy-mediated diffusion in crystalline solids have mainly been observed for diffusion in $\mathrm{Zr}$ rich $\mathrm{Co}-\mathrm{Zr}$ and $\mathrm{Ni}-\mathrm{Zr}$ metallic glasses. They have tentatively been attributed to the formation of diffusion-mediating thermal defects which are delocalized. On the other hand, very recent molecular-dynamics simulations for $\mathrm{Ni}-\mathrm{Zr}$ alloys (Sec. IX) are in reasonable agreement with the microscopic picture that transport in metallic glasses takes place by the thermally activated motion of chains of atoms, where a large fraction of the observed activation volume is the migration volume of the chains.

Isotope-effect experiments play a prominent role in demonstrating the collective nature of diffusion processes in metallic glasses (Sec. VII). The almost vanishing isotope effects measured in relaxed conventional metallic glasses provide strong evidence for highly collective hopping mechanisms, as confirmed by moleculardynamics simulations. Isotope-effect measurements on bulk metallic glasses in the supercooled melt and in the glassy state yielded very small and temperatureindependent values similar to the isotope effects for conventional metallic glasses. These observations strongly support the view that highly collective, thermally activated hopping processes, as occurring in the glassy state, also determine long-range diffusion in the deeply supercooled melt above the caloric glass transition.

Nonvanishing isotope effects for an as-quenched metal-metalloid glass similar to those observed for vacancy-mediated diffusion in metal crystals lend support to the view that during diffusion annealing of unrelaxed glasses, quenched-in quasivacancies serve as diffusion vehicles until they have annealed out. No change in the isotope effect has been observed, however, during structural relaxation of thin films of amorphous transition-metal alloys.

Diffusion studies under particle irradiation proved to be a useful tool in elucidating diffusion mechanisms in crystalline solids and have been performed on metallic glasses as well (Sec. VIII). Of particular interest are diffusion experiments under low-transferred-energy irradiation, which lead to an increase in the preexponential factors of the diffusion coefficients, whereas the activation enthalpies under irradiation and in the absence of irradiation are the same. As demonstrated in moleculardynamics simulations of radiation-induced diffusion, diffusional atomic displacements are favored in damaged regions. Molecular-dynamics simulations of diffusion processes in undercooled melts and metallic glasses (Sec. IX) have contributed significantly to the atomic understanding of diffusion processes. With decreasing temperature, transport of matter changes from liquidlike viscous flow (via atomic collisions) to thermally activated transport characteristic of solids. In glass-forming alloys this transition takes place in two steps. According to the mode-coupling theory, at a critical temperature $T_{c}$ the dynamics of the supercooled melt changes from a high-temperature homogeneous viscous flow to a pseudoarrested low-temperature flow. This is supported by the observation that the Stokes-Einstein relation between diffusivity and viscosity is fulfilled neither for conventional nor for bulk metallic glasses and their supercooled melts. Already well above $T_{c}$ the dynamics starts to become heterogeneous in the form of correlated motion of chains or rings of atoms. Upon further cooling well below $T_{c}$ the caloric glass transition occurs at $T_{g}$. Starting below $T_{c}$, diffusion follows the classical Arrhenius relationship. As mentioned before, however, the activation energy is an effective quantity, affected by structural changes above $T_{g}$. The pertinent thermally activated processes are generally envisaged as being highly collective and involving many atoms.

Computer simulations indicate a connection between low-frequency excitations in glasses and long-range diffusion. In the amorphous state, isolated jump events can be identified in molecular-dynamics simulations. At the lowest temperatures accessible to simulations, the dynamics in amorphous solids is characterized by local excitations. Local soft vibrations provide a way to attain large atomic amplitudes, which is a prerequisite for at- 
oms to overcome barriers. Collective atomic motion occurs in a chainlike manner that leads to a total displacement of the order of the nearest-neighbor distance. Such chains typically involve between 10 and 20 atoms, where each atom moves only a small fraction of the nearestneighbor distance. With increasing temperature, the total jump length, the jump length of single atoms, and the number of atoms involved in such displacement chains increase. With further increasing temperature these collective events become more and more frequent and finally merge into flow. Beyond this, the recent long-time simulations for temperatures close to the experimental $T_{g}$ indicate that correlated motions of local groups of chains in the form of chain avalanches may introduce an additional type of process into the dynamics of the glassy state.

The experiments and molecular-dynamics simulations discussed in this review clearly show that diffusion in metallic glasses contrasts sharply with diffusion in crystalline metals. It requires completely different concepts based on thermally activated highly collective hopping processes. The same solidlike processes have also been demonstrated to govern diffusion in the supercooled liquid state. This strongly supports the central prediction of the mode-coupling theory that liquidlike flow processes freeze in well above the caloric glass transition temperature.

\section{ACKNOWLEDGMENTS}

The authors are indebted to many co-workers who have made valuable contributions to the present subject, in particular to B. Boeddeker, D. Caprion, H. Ehmler, P. Fielitz, C. Gaukel, M. Guerdane, A. Heesemann, M. Kluge, P. Klugkist, K. Knorr, C. Oligschleger, A. Rehmet, U. Rößler, P. Schaaff, P. Scharwaechter, T. Schuler, F. Wenwer, V. Zöllmer, and Th. Zumkley. Financial support by the Deutsche Forschungsgemeinschaft within the framework of the program "Unterkühlte Metallschmelzen-Phasenselektion und Glasbildung" is gratefully acknowledged. Special thanks are given to Dieter Herlach, the speaker of the program. The program has benefited greatly from his competence and efforts.

\section{LIST OF SYMBOLS}

Quantity Explanation

\begin{tabular}{ll}
\hline$a$ & Lattice parameter \\
$C(x, t)$ & Concentration at position $x$ and time $t$ \\
$c_{D}$ & Diffusion-defect concentration \\
$D$ & Diffusion coefficient \\
$D_{0}$ & Preexponential factor in the Arrhenius law \\
$E$ & Isotope-effect parameter \\
$f$ & Correlation factor \\
$F_{0}$ & Volume fraction of the $\delta$ phase prior to irra- \\
$g$ & diation \\
$G$ & Geometrical factor \\
$H$ & Self-part of the van Hove function \\
& Activation enthalpy of diffusion
\end{tabular}

$\begin{array}{ll}H^{\text {irr }} & \text { Activation enthalpy under irradiation } \\ k_{B} & \text { Boltzmann's constant } \\ m_{a} & \text { Mass of isotope } \alpha \\ m+M & \text { Effective mass } \\ p & \text { (Hydrostatic) pressure } \\ P(r, t) & \begin{array}{l}\text { Probability that an atom has moved over a } \\ \text { distance } r \text { during a time } t\end{array} \\ \boldsymbol{R}^{n} & \text { Position of atom } n \\ S_{0} & \text { Tracer atoms per unit area } \\ T & \text { Absolute temperature } \\ T_{c} & \text { Critical temperature of mode-coupling } \\ & \text { theory } \\ T_{g} & \text { Glass transition temperature or glass trans- } \\ & \text { formation temperature } \\ T_{f} & \text { Fictive temperature } \\ T_{x} & \text { Crystallization temperature } \\ V_{\text {act }} & \text { Activation volume } \\ V^{F} & \text { Formation volume } \\ V^{M} & \text { Migration volume } \\ \Delta S & \text { Entropy of diffusion } \\ \Delta Z & \text { Valence difference } \\ \Omega & \text { Atomic volume } \\ \sqrt{D t} & \text { Typical diffusion length } \\ \Delta D & \text { Diffusivity enhancement } \\ v_{0} & \text { Attempt frequency } \\ \Phi & \text { Irradiation-particle flux } \\ \sigma & \text { Cross section for the production of } \delta \text { spots } \\ \eta & \text { Viscosity } \\ \Delta U & \text { Barrier height increase under hydrostatic } \\ \left\langle u^{2}(t)\right\rangle & \text { pressure } p \\ \left\langle u^{4}(t)\right\rangle & \text { Mean quadratic displacement } \\ \beta_{\mu} & \text { Mean quartic displacement } \\ \mu & \text { Temperature dependence of shear modulus } \\ \tau_{D} & \text { Shear modulus } \\ \tau_{\eta} & \text { Characteristic diffusion time } \\ & \text { Matrix relaxation time } \\ & \end{array}$

\section{REFERENCES}

Akhtar, D., B. Cantor, and R. W. Cahn, 1982, Acta Metall. 30, 1571.

Allen, M. P., and D. J. Tildesley, 1987, Computer Simulation of Liquids (Oxford University, Oxford).

Almazouzi, A., M.-P. Macht, V. Naundorf, and G. Neumann, 1996, Phys. Rev. B 54, 857.

Almazouzi, A., M.-P. Macht, V. Naundorf, and G. Neumann, 1998, Phys. Status Solidi A 167, 15.

Angell, C. A., 1995, Science 267, 1924.

Averback, R. S., 1991, Mater. Res. Bull. 16, 47.

Averback, R. S., and H. Hahn, 1988, Phys. Rev. B 37, 10383.

Averback, R. S., and H. Hahn, 1989, Mater. Sci. Forum 37, 245.

Averback, R. S., H. Hahn, and Fu-Rong Ding, 1988, J. LessCommon Met. 140, 267.

Balucani, U., and M. Zoppi, 1994, Dynamics of the Liquid State (Clarendon, Oxford).

Barrett, C. S., and T. B. Massalski, 1966, Eds., Materials Science and Engineering Series (McGraw-Hill, New York).

Baskes, M. I., and R. A. Johnson, 1994, Modell. Simul. Mater. Sci. Eng. 2, 147.

Berry, B. S., and W. G. Pritchet, 1981, Phys. Rev. B 24, 2299.

Birac, C., and D. Lesueur, 1976, Phys. Status Solidi A 36, 247. Böddeker, B., 1999, Ph.D. thesis (University of Göttingen). 
Böddeker, B., and H. Teichler, 1999, Phys. Rev. E 59, 1948.

Borg, R. J., and G. J. Dienes, 1988, Eds., An Introduction to Solid State Diffusion (Academic, Boston).

Bøttiger, J., K. Dyrbye, K. Pampus, B. Torp, and P. H. Wiene, 1988, Phys. Rev. B 37, 9951.

Bøttiger, J., K. Pampus, and B. Torp, 1987, Europhys. Lett. 4, 915.

Brandt, E. H., 1989, J. Phys.: Condens. Matter 1, 9985.

Buchenau, U., Y. M. Galperin, V. L. Gurevich, and H. R. Schober, 1991, Phys. Rev. B 43, 5039.

Buchenau, U., M. Prager, N. Nücker, A. J. Dianoux, N. Ahmad, and W. A. Phillips, 1986, Phys. Rev. B 34, 5665.

Buchenau, U., H. M. Zhou, N. Nücker, K. S. Gilroy, and W. A. Philips, 1988, Phys. Rev. Lett. 60, 1318.

Budke, E., P. Fielitz, M.-P. Macht, V. Naundorf, and G. Frohberg, 1997, Defect Diffus. Forum 143-147, 825.

Busch, R., and W. L. Johnson, 1998, Mater. Sci. Forum 269272, 577.

Busch, R., A. Masuhr, E. Bakke, T. A. Waniuk, and W. L. Johnson, 1999, in Bulk Metallic Glasses, edited by W. L. Johnson, C. T. Liu, and A. Inoue, MRS Symposia Proceedings No. 554 (Materials Research Society, Pittsburgh), p. 223. Cahn, R. W., 1983, in Physical Metallurgy, edited by R. W. Cahn and P. Haasen (Elsevier Science, Amsterdam), p. 385. Cahn, R. W., 1991, in Materials Science and Technology, edited by R. W. Cahn, P. Hassen, and E. J. Kramer (VCH, Weinheim), p. 403.

Cahn, R. W., J. E. Evetts, J. Patterson, R. E. Somekh, and C. K. Jackson, 1980, J. Mater. Sci. 15, 702.

Cantor, B., 1985, in Proceedings of the 5th International Conference on Rapidly Quenched Metals, edited by S. Steeb and H. Warlimont (Elsevier Science, Amsterdam), p. 595.

Cantor, B., 1986, in Amorphous Metals and Semiconductors, edited by P. Haasen and R. I. Jaffee (Pergamon, Oxford), p. 108.

Cantor, B., and R. W. Cahn, 1983, in Amorphous Metallic Alloys, edited by F. E. Luborsky (Butterworth, London), p. 487. Caprion, D., J. Matsui, and H. R. Schober, 2000, Phys. Rev. Lett. 85, 4293.

Chen, H. S., 1974, Acta Metall. 22, 1505.

Chen, H. S., 1978, J. Appl. Phys. 49, 3289.

Chen, H. S., 1983, in Amorphous Metallic Alloys, edited by F. E. Luborsky (Butterworth, London), p. 169.

Chen, H. S., L. C. Kimerling, J. M. Poate, and W. L. Brown, 1978, Appl. Phys. Lett. 32, 461.

Clement, W., R. H. Willens, and P. Duwez, 1960, Nature (London) 187, 869 .

Cohen, M. H., and G. S. Grest, 1981, Ann. N.Y. Acad. Sci. 271, 199.

Cohen, M. H., and D. J. Turnbull, 1959, Chem. Phys. 31, 1164. Crank, J., 1975, The Mathematics of Diffusion (Oxford University, Oxford).

Dariel, M. P., 1974, Scr. Metall. 8, 869.

Davies, H. A., 1983, in Amorphous Metallic Alloys, edited by F. E. Luborsky (Butterworth, London), Chap. 2.

Dederichs, P. H., C. Lehmann, H. R. Schober, A. Scholz, and R. Zeller, 1978, J. Nucl. Mater. 69-70, 176.

Donati, C., J. F. Douglas, W. Kob, S. J. Plimpton, P. H. Poole, and S. C. Glotzer, 1998, Phys. Rev. Lett. 80, 2338.

Dörner, W., and H. Mehrer, 1991, Phys. Rev. B 44, 101.

Drehmann, A. L., L. A. Greer, and D. Turnbull, 1982, Appl. Phys. Lett. 41, 716.
Duine, P. A., J. Sietsma, and A. van den Beukel, 1992, Acta Metall. Mater. 40, 743.

Duine, P. A., J. Sietsma, and A. van den Beukel, 1993, Phys. Rev. B 48, 6957.

Duine, P. A., J. Sietsma, and A. van den Beukel, 1995, Defect Diffus. Forum 117-118, 91.

Duine, P. A., S. K. Wonnell, and J. Sietsma, 1994, Mater. Sci. Eng., A 179-180, 270.

Egami, T., 1981, Ann. N.Y. Acad. Sci. 371, 238.

Egami, T., K. Maeda, and V. Vitek, 1980, Philos. Mag. A 41, 883.

Ehmler, H., A. Heesemann, K. Rätzke, F. Faupel, and U. Geyer, 1998, Phys. Rev. Lett. 80, 4919.

Ehmler, H., K. Rätzke, and F. Faupel, 1999, J. Non-Cryst. Solids 250-252, 684.

Faupel, F., 1992, Phys. Status Solidi A 134, 9.

Faupel, F., P. W. Hüppe, and K. Rätzke, 1990, Phys. Rev. Lett. 65, 1219.

Faupel, F., P. W. Hüppe, K. Rätzke, R. Willecke, and T. Hehenkamp, 1992, J. Vac. Sci. Technol. A 10, 92.

Faupel, F., K. Rätzke, H. Ehmler, P. Klugkist, V. Zöllmer, C. Nagel, A. Rehmet, and A. Heesemann, 2001, in Supercooled Liquid, Bulk Glassy, and Nanocrystalline State of Alloys, edited by A. Inoue, A. Yavari, W. C. Johnson, and R. Dauskardt, MRS Symposia Proceedings No. 664 (Materials Research Society, Pittsburgh), p. L2.1.1.

Faupel, F., K. Rätzke, and P. W. Hüppe, 1993, Defect Diffus. Forum 95-98, 1175.

Fedorov, G. B., 1970, in Mobility of Atoms in Crystal Lattices, edited by V. N. Svechnikov (translated from Russian by Israel Programme for Scientific Translations, Jerusalem), p. 28.

Fehr, T., and H. Löwen, 1995, Phys. Rev. E 52, 4016.

Fielitz, P., M.-P. Macht, V. Naundorf, and G. Frohberg, 1999, J. Non-Cryst. Solids 250-252, 674.

Frank, W., 1989, Defect Diffus. Forum 66-69, 387.

Frank, W., 1997, Defect Diffus. Forum 143-147, 695.

Frank, W., U. Gösele, and A. Seeger, 1980, in Radiaton Physics of Semiconductors and Related Materials, 1979, edited by G. P. Kekelidze and V. I. Shakhovtsov (Tbilisi State University, Tbilisi), p. 110.

Frank, W., U. Hamlescher, H. Kronmüller, P. Scharwaechter, and T. Schuler, 1996, Phys. Scr. T66, 201.

Frank, W., A. Hörner, P. Scharwaechter, and H. Kronmüller, 1994, Mater. Sci. Eng. A 179-180, 36.

Frank, W., J. Horváth, and H. Kronmüller, 1988, Mater. Sci. Eng. 97, 415.

Frank, W., A. Strohm, and T. Voss, 2003, in Proceedings of the 2nd International Conference on Mass and Charge Transport in Inorganic Materials, edited by P. Vincenzini and V. Buscaglia (Techna, Faenza), (in press).

Frohberg, G., K.-H. Kraatz, and H. Wever, 1987, Mater. Sci. Forum 15-18, 529.

Gärtner, F., C. Michaelsen, and R. Bormann, 1997, Philos. Mag. B 76, 511.

Gaukel, C., M. Kluge, and H. R. Schober, 1999, Philos. Mag. 79, 1907.

Gaukel, C., and H. R. Schober, 1998, Solid State Commun. 107, 1 .

Gerling, R., F. P. Schimansky, and R. Wagner, 1985, in Proceedings of the 5th International Conference on Rapidly Quenched Metals, edited by S. Steeb and H. Warlimont (Elsevier Science, Amsterdam), p. 1377. 
Geschneidner, K. A., Jr., 1964, in Solid State Physics Vol. 16, edited by F. Seitz and D. Turnbull (Academic, New York), p. 276.

Geyer, U., W. L. Johnson, S. Schneider, Y. Qiu, T. A. Tombrello, and M.-P. Macht, 1996, Appl. Phys. Lett. 69, 2492.

Geyer, U., A. Rehmet, and S. Schneider, 1999, in Bulk Metallic Glasses, edited by W. L. Johnson, C. T. Liu, and A. Inoue, MRS Symposia Proceedings No. 554 (Materials Research Society, Pittsburgh), p. 275.

Geyer, U., S. Schneider, W. L. Johnson, Y. Qiu, T. A. Tombrello, and M.-P. Macht, 1995, Phys. Rev. Lett. 75, 2364.

Götze, W., 1999, J. Phys.: Condens. Matter 11, A1.

Götze, W., and L. Sjögren, 1991, J. Non-Cryst. Solids 131-133, 161.

Götze, W., and A. Sjölander, 1992, Rep. Prog. Phys. 55, 241. Grandjean, A., P. Blanchard, and Y. Limoge, 1997, Phys. Rev. Lett. 78, 697.

Greer, A. L., 1999, Nature (London) 402, 132.

Greer, A. L., C. J. Lin, and F. Spaepen, 1982, in Proceedings of the 4th International Conference on Rapidly Quenched Metals, edited by T. Masumoto and K. Suzuki (Japan Institute of Metals, Sendai), p. 567.

Guerdane, M., 2000, Ph.D. thesis (University of Göttingen). Gupta, D., K. N. Tu, and K. W. Asai, 1975, Phys. Rev. Lett. 35, 796.

Haasen, P., 1986, in Amorphous Metals and Semiconductors, edited by P. Haasen and R. I. Jaffee (Pergamon, Oxford), p. 108.

Hahn, H., R. S. Averback, T. Diaz de la Rubia, and P. R. Okamoto, 1986, in Beam-Solid Interactions and Phase Transformations, edited by H. Kurz, G. L. Olson, and J. M. Poate, MRS Symposia Proceedings No. 51 (Materials Research Society, Pittsburgh), p. 491.

Hahn, H., R. S. Averback, Fu-Rong Ding, C. Loxton, and J. Baker, 1987, Mater. Sci. Forum 15-18, 511.

Hahn, H., R. S. Averback, and H. M. Shyu, 1988, J. LessCommon Met. 140, 345.

Hamlescher, U., 1994, Diploma thesis (Stuttgart University).

Hansen, M., and K. Anderko, 1958, in Metallurgy and Metallurgical Engineering Series (McGraw-Hill, New York).

Hausleitner, Ch., and J. Hafner, 1992, Phys. Rev. B 45, 115.

He, Y., R. B. Schwarz, D. Mandrus, and L. Jacobson, 1996, J. Non-Cryst. Solids 205-207, 602.

Hearmon, R. F. S., 1979, in The Elastic Constants of Crystals and Other Anisotropic Materials, Landolt-Börnstein, New Series, Group III, Vol. 11, Part 1, edited by K.-H. Hellwege and A. M. Hellwege (Springer, Berlin), p. 9.

Heesemann, A., K. Rätzke, F. Faupel, J. Hoffmann, and K. Heinemann, 1995, Europhys. Lett. 29, 221.

Heesemann, A., K. Rätzke, V. Zöllmer, and F. Faupel, 2000, Z. Metallkd. 91, 909.

Heesemann, A., K. Rätzke, V. Zöllmer, and F. Faupel, 2001, New J. Phys. 3, 6.1.

Heesemann, A., V. Zöllmer, K. Rätzke, and F. Faupel, 2000, Phys. Rev. Lett. 84, 1467.

Hilzinger, R., and K. Reichert, 1999, in Proceedings of the International Conference on High Frequency Magnetic Materials 1999 (Gorham Intertech, Santa Clara, CA).

Höfler, H. J., R. S. Averback, G. Rummel, and H. Mehrer, 1992, Philos. Mag. Lett. 66, 301.

Hood, G. M., 1978, J. Phys. F: Met. Phys. 8, 1677.

Hörner, A., 1990, Diploma thesis (Stuttgart University).

Hörner, A., 1993, Ph.D. thesis (Stuttgart University).
Horváth, J., 1990, in Diffusion in Solid Metals and Alloys, Landolt-Börnstein, New Series, Group III, Vol. 26, edited by H. Mehrer (Springer, Berlin), p. 437.

Horváth, J., K. Freitag, and H. Mehrer, 1985, in Proceedings of the 5th International Conference on Rapidly Quenched Metals, edited by S. Steeb and H. Warlimont (Elsevier Science, Amsterdam), p. 751.

Horváth, J., and H. Mehrer, 1986, Cryst. Lattice Defects Amorphous Mater. 13, 1.

Horváth, J., J. Ott, K. Pfahler, and W. Ulfert, 1988, Mater. Sci. Eng. 97, 409.

Horváth, J., K. Pfahler, W. Ulfert, W. Frank, and H. Kronmüller, 1987, Mater. Sci. Forum 15-18, 523.

Horváth, J., K. Pfahler, W. Ulfert, W. Frank, and H. Mehrer, 1985, J. Phys. (Paris) 46, C8-645.

Hoshino, K., R. S. Averback, H. Hahn, and S. J. Rothman, 1988, Defect Diffus. Forum 59, 225.

Hüppe, P.-W., and F. Faupel, 1992, Phys. Rev. B 46, 120.

Inoue, A., 1995, Mater. Trans., JIM 36, 866.

Inoue, A., 1998, Bulk Amorphous Alloys (Trans Tech Publications, Zürich).

Inoue, K., T. Kanaya, S. Ikeda, D. Kaji, K. Shibata, M. Misawa, and Y. Kiyanagi, 1991, J. Chem. Phys. 95, 5332.

Inoue, A., T. Nakamura, N. Nishiyama, and T. Masumoto, 1992, Mater. Trans., JIM 33, 937.

Inoue, A., T. Zhang, and T. Masumoto, 1990a, Mater. Trans., JIM 30, 965.

Inoue, A., T. Zhang, and T. Masumoto, 1990b, Mater. Trans., JIM 31, 177.

Jäckle, J., 1986, Rep. Prog. Phys. 49, 171.

Johnson, W. L., 1999a, in Bulk Metallic Glasses, edited by W. L. Johnson, C. T. Liu, and A. Inoue, MRS Symposia Proceedings No. 554 (Materials Research Society, Pittsburgh), p. 311.

Johnson, W. L., 1999b, Mater. Res. Bull. 11, 104.

Kijek, M., D. W. Palmer, and B. Cantor, 1986, Acta Metall. 34, 1455.

Kimura, M., and F. Yonezawa, 1983, in Topological Disorder in Condensed Matter, edited by F. Yonezawa and T. Niniomiya (Springer, Berlin), p. 80.

Kirchheim, R., F. Sommer, and G. Schluckebier, 1982, Acta Metall. 30, 1059.

Kirkaldy, J. S., and D. J. Young, 1987, Diffusion in the Condensed State (Institute of Metals, London).

Kluge, M., 2001, Ph.D. thesis (University of Aachen).

Kluge, M., and H. R. Schober, 2000, Phys. Rev. E 62, 597.

Kluge, M., and H. R. Schober, 2001, Defect Diffus. Forum 194-199, 849.

Klugkist, P., K. Rätzke, and F. Faupel, 1998, Phys. Rev. Lett. 81, 614.

Klugkist, P., K. Rätzke, F. Faupel, P. Troche, P. Fielitz, and V. Naundorf, 1999, Philos. Mag. Lett. 79, 827.

Klugkist, P., K. Rätzke, S. Rehders, P. Troche, and F. Faupel, 1998, Phys. Rev. Lett. 80, 3288.

Knorr, K., M.-P. Macht, K. Freitag, and H. Mehrer, 1999, J. Non-Cryst. Solids 250, 669.

Knorr, K., M.-P. Macht, and H. Mehrer, 1999, in Bulk Metallic Glasses, edited by W. L. Johnson, C. T. Liu, and A. Inoue, MRS Symposia Proceedings No. 554 (Materials Research Society, Pittsburgh), p. 269.

Knorr K., M.-P. Macht, and H. Mehrer, 2000, in Materials Development and Processing-Bulk Amorphous Materials, Undercooling and Powder Metallurgy, edited by J. V. Wood, L. Schultz, and D. M. Herlach (VCH, Weinheim), p. 22. 
Kob, W., and H. Andersen, 1995, Phys. Rev. E 51, 4626.

Koiwa, M., H. Nakajima, and K.-L. Hirano, 1993, Eds., Proceedings of the International Conference on Diffusion in Materials-DIMAT-92, Kyoto 1992 (Trans Tech Publications, Brookfield), Defect Diffus. Forum 95-98, 1.

Köster, U., and U. Herold, 1980, J. Phys. (Paris) 41, C8-352.

Köster, U., U. Herold, and A. Becker, 1981, in Proceedings of the 4th International Conference on Rapidly Quenched Metals, edited by T. Masumoto and K. Suzuki (Japanese Institute of Metals, Sendai), p. 587.

Kronmüller, H., 1998, in Elements of Rapid Solidification: Fundamentals and Application, Springer Series in Materials Sciences Vol. 29, edited by M. A. Otooni (Springer, Berlin), p. 93.

Kronmüller, H., and W. Frank, 1989, Radiat. Eff. Defects Solids 108, 81 .

Kui, H. W., A. L. Greer, and D. Turnbull, 1984, Appl. Phys. Lett. 45, 615 .

Laird, B. B., and H. R. Schober, 1991, Phys. Rev. Lett. 66, 636. Lazarus, D., 1960, in Diffusion in Metals, Solid State Physics Vol. 10, edited by F. Seitz and D. Turnbull (Academic, New York), p. 71.

Le Claire, A. D., 1962, Philos. Mag. 7, 141.

Le Claire, A. D., 1966, Philos. Mag. 14, 1271.

Le Claire, A. D., 1978, J. Nucl. Mater. 69-70, 70.

Le Claire, A. D., and G. Neumann, 1990, in Diffusion in Solid Metals and Alloys, Landolt-Börnstein, New Series, Group III, Vol. 26, edited by H. Mehrer (Springer, Berlin), p. 85.

Lewis, L. J., 1991, Mater. Sci. Eng., A 133, 423.

Limoge, Y., 1992a, Scr. Metall. Mater. 26, 809.

Limoge, Y., 1992b, Defect Diffus. Forum 83, 145.

Limoge, Y., and J. L. Bocquet, 1990, Phys. Rev. Lett. 65, 60.

Limoge, Y., and G. Brebec, 1988, Acta Metall. 36, 665.

Limoge, Y., G. Brebec, and Y. Adda, 1983, in Diffusion in Metals and Alloys-DIMETA-82, edited by F. J. Kedves and D. L. Beke (Trans Tech Publications, Zürich), p. 285.

Loirat, Y., J. L. Bocquet, and Y. Limoge, 2000, J. Non-Cryst. Solids 265, 252.

Luborsky, F. E., 1983, Amorphous Metallic Alloys (Butterworth Monographs in Materials, London).

Masuhr, A., T. A. Waniuk, R. Busch, and W. L. Johnson, 1999, Phys. Rev. Lett. 82, 2290.

Mehrer, H., 1987, Phys. Status Solidi A 104, 247.

Mehrer, H., 1990, Ed., Diffusion in Solid Metals and Alloys, Landolt-Börnstein, New Series, Group III, Vol. 26 (Springer, Berlin), p. 1.

Mehrer, H., 1996, Defect Diffus. Forum 129-130, 57.

Mehrer, H., and W. Dörner, 1989, Defect Diffus. Forum 66-69, 189.

Mehrer, H., Ch. Herzig, N. A. Stolwijk, and H. Bracht, 1997, Eds., Proceedings of the International Conference on Diffusion in Materials-DIMAT-96 (Scitec Publications, Nordkirchen).

Mehrer, H., and F. Wenwer, 1998, in Diffusion in Condensed Matter, edited by J. Kärger, P. Heitjans, and R. Habenlandt (Vieweg, Wiesbaden), p. 1.

Meyer, A., J. Wuttke, W. Petry, A. Peker, R. Bormann, G. Coddens, L. Kranich, O. G. Randl, and H. Schober, 1996, Phys. Rev. B 53, 12107.

Meyer, A., J. Wuttke, W. Petry, O. G. Randl, and H. Schober, 1998, Phys. Rev. Lett. 80, 4454.

Mihara, T., S. Otake, H. Fukushima, and M. Doyama, 1981, J. Phys. F: Met. Phys. 11, 727.
Miyagawa, H., Y. Hiwatari, B. Bernu, and J. P. Hansen, 1988, J. Chem. Phys. 88, 3879.

Mullen, J. G., 1961, Phys. Rev. 121, 1649.

Müller, A., V. Naundorf, and M.-P. Macht, 1988, J. Appl. Phys. 64, 3445 .

Nachtrieb, N. H., 1976, Ber. Bunsenges. Phys. Chem. 80, 678.

Nagel, C., K. Rätzke, E. Schmidtke, F. Faupel, and W. Ulfert, 1999, Phys. Rev. B 60, 9212.

Nagel, C., K. Rätzke, E. Schmidtke, J. Wolff, U. Geyer, and F. Faupel, 1998, Phys. Rev. B 57, 10224.

Nakajima, H., T. Kojima, T. Zumkley, N. Nishiyama, and A. Inoue, 1999, in Proceedings of the International Conference on Solid-Solid Phase Transformation, edited by M. Koiwa, K. Otsuka, and T. Miyazaki (Japanese Institute of Metals, Sendai), p. 441.

Nakajima, H., W. Sprengel, and K. Nonaka, 1997, Defect Diffus. Forum 143-147, 803.

Naundorf, V., M.-P. Macht, A. S. Bakai, and N. Lazarev, 1998, J. Non-Cryst. Solids 224, 122.

Naundorf, V., M.-P. Macht, A. S. Bakai, and N. Lazarev, 1999, J. Non-Cryst. Solids 250-252, 679.

Neumann, G., 1983, Impurity Diffusion in Pure Metals, Diffusion and Defect Monograph Series No. 7 (Trans Tech, Zürich), p. 3.

Neumann, G., and V. Tölle, 1995, Philos. Mag. 71, 231.

Nonaka, H., Y. Kimura, K. Yamauchi, H. Nakijama, T. Zhang, A. Inoue, and T. Masumoto, 1997, Defect Diffus. Forum 143147, 837.

Oligschleger, C., and H. R. Schober, 1999, Phys. Rev. B 59, 811. Park, J.-Y., and G. B. McKenna, 2000, Phys. Rev. B 61, 6667.

Pearson, W. B., 1958, in International Series of Monographs on Metal Physics and Physical Metallurgy Vol. 4, edited by G. V. Reynor (Pergamon, New York).

Peker, A., and W. L. Johnson, 1993, Appl. Phys. Lett. 63, 2342. Pfahler, K., J. Horváth, W. Frank, and H. Mehrer, 1985, in Proceedings of the 5th International Conference on Rapidly Quenched Metals, edited by S. Steeb and H. Warlimont (Elsevier Science, Amsterdam), p. 755.

Philibert, J., 1991, Diffusion and Mass Transport in Solids (Les Editions de Physique, Les Ulis).

Rahman, A., 1964, Phys. Rev. A 136, 405.

Rank, C., J. Horváth, M. Werner, and W. Frank, 1990, Vak. Prax. 2, 286.

Rätzke, K., and F. Faupel, 1991, Scr. Metall. 25, 2233.

Rätzke, K., and F. Faupel, 1992, Phys. Rev. B 45, 7459.

Rätzke, K., and F. Faupel, 1995, J. Non-Cryst. Solids 181, 261.

Rätzke, K., A. Heesemann, and F. Faupel, 1995, J. Phys.: Condens. Matter 7, 7633.

Rätzke, K., P. W. Hüppe, and F. Faupel, 1992, Phys. Rev. Lett. 68, 2347.

Rätzke, K., P. Klugkist, and F. Faupel, 1999, Defect Diffus. Forum 165-166, 43.

Rätzke, K., P. Klugkist, A. Heesemann, and F. Faupel, 2001, Defect Diffus. Forum 194-199, 833.

Rehmet, A., K. Rätzke, F. Faupel, P. D. Eversheim, K. Freitag, U. Geyer, and S. Schneider, 2001, Appl. Phys. Lett. 79, 2892.

Richter, D., A. Dianoux, W. Petry, 1989, Dynamics of Disordered Materials (Springer, Heidelberg).

Rosenblum, M. P., F. Spaepen, and D. Turnbull, 1980, Appl. Phys. Lett. 37, 184.

Rößler, U. K., and H. Teichler, 2000, Phys. Rev. E 61, 394.

Rothman, S. J., 1984, in Diffusion in Crystalline Solids, edited by G. E. Murch and A. S. Nowick (Academic, New York). 
Roux, J. N., J. L. Barrat, and J. P. Hansen, 1989, Phys. Rev. A 38, 454.

Rummel, G., and H. Mehrer, 1994, Phys. Status Solidi A 185, 327.

Schaaff, P., 2001, Ph.D. thesis (Stuttgart University).

Scharwaechter, P., 1993, Ph.D. thesis (Stuttgart University).

Scharwaechter, P., W. Frank, and H. Kronmueller, 1996a, Z. Metallkd. 87, 885.

Scharwaechter, P., W. Frank, and H. Kronmueller, 1996b, Z. Metallkd. 87, 892.

Scheidler, P., W. Kob, and K. Binder, 2000, Europhys. Lett. 52, 277.

Schilling, W., 1978, J. Nucl. Mater. 69-70, 465.

Schneider, S., P. Thiagarajan, U. Geyer, and W. L. Johnson, 1997, in Structure and Dynamics of Glasses and Glass Formers, edited by C. A. Angell, K. L. Ngai, J. Kieffer, T. Egami, and G. U. Nienhaus, MRS Symposia Proceedings No. 455 (Materials Research Society, Pittsburgh), p. 295.

Schober, H. R., 1993, Physica A 201, 14.

Schober, H. R., 2001, Solid State Commun. 119, 73.

Schober, H. R., 2002, Phys. Rev. Lett. 88, 145901.

Schober, H. R., C. Gaukel, and C. Oligschleger, 1997, Prog. Theor. Phys. Suppl. 126, 67.

Schober, H. R., and C. Oligschleger, 1996, Phys. Rev. B 53, 11469.

Schober, H. R., C. Oligschleger, and B. B. Laird, 1993, J. NonCryst. Solids 156-158, 965.

Schoen, A., 1958, Phys. Rev. Lett. 1, 138.

Schoo, U., C. Cramer, E. Ratai, and H. Mehrer, 2001, Defect Diffus. Forum 194-199, 925.

Schoo, U., and H. Mehrer, 2000, Solid State Ionics 130, 243.

Schrøder, B. T., and J. C. Dyre, 1998, J. Non-Cryst. Solids 235237, 331.

Schuehmacher, J. J., and P. Guiraldenq, 1983, Acta Metall. 31, 2043.

Schuler, T., 2001, Ph.D. thesis (Stuttgart University).

Schuler, T., U. Hamlescher, P. Scharwaechter, and W. Frank, 1997, Defect Diffus. Forum 143-147, 753.

Schuler, T., P. Scharwaechter, and W. Frank, 1999, in Microstructural Processes in Infrared Materials, edited by S. J. Zinkle, G. Lucas, R. Ewing, and J. Williams, MRS Symposia Proceedings No. 540 (Materials Research Society, Pittsburgh), p. 195.

Seeger, A., and K. P. Chik, 1968, Phys. Status Solidi 29, 455.

Sharma, S. K., S. Banerjee, Kuldeep, and A. K. Jain, 1988, Acta Metall. Mater. 36, 1683.

Sharma, S. K., S. Banerjee, Kuldeep, and A. K. Jain, 1989, J. Mater. Res. 4, 603.

Sharma, S. K., and F. Faupel, 1999, J. Mater. Res. 14, 3200.

Sharma, S. K., Kuldeep, and A. K. Jain, 1988, Mater. Sci. Eng. 100, 145.

Sharma, S. K., M.-P. Macht, and V. Naundorf, 1992, Acta Metall. Mater. 40, 2439.

Sharma, S. K., M.-P. Macht, and V. Naundorf, 1993, J. NonCryst. Solids 156-158, 437.

Sharma, S. K., M.-P. Macht, and V. Naundorf, 1994, Phys. Rev. B 49, 6655.

Sharma, S. K., and P. Mukhopadhyay, 1990, Acta Metall. Mater. 38, 129.

Shelby, J. E., 1997, Introduction to Glass Science and Technology (Royal Society of Chemistry, Cambridge).

Shewmon, P. G., 1963, Diffusion in Solids (McGraw-Hill, New York).
Shewmon, P. G., 1989, Diffusion in Solids (Minerals, Metals, and Materials Society, Warrendale).

Shimoji, M., and T. Itami, 1986, Atomic Transport in Liquids and Metals (Trans Tech Publications, Aedermannsdorf).

Sizmann, R., 1978, J. Nucl. Mater. 69-70, 386.

Sjögren, L., 1991, J. Phys. B 79, 5.

Spaepen, F., 1981, in Physics of Defects, edited by R. Balian, M. Kléman, and J. P. Poirer (North-Holland., Amsterdam), p. 135.

Spaepen, F., 1988, Mater. Sci. Eng. 97, 403.

Stelter, E. C., and D. Lazarus, 1987, Phys. Rev. B 36, 9545.

Steyer, M., H. U. Krebs, and H. C. Freyhardt, 1987, Z. Phys. B: Condens. Matter 66, 317.

Suck, J.-B., and H. Rudin, 1983, in Glassy Metals II, edited by H. Beck and H.-J. Güntherodt, Topics in Applied Physics No. 53 (Springer, Berlin), p. 217.

Tang, X. P., R. Busch, W. L. Johnson, and Y. Wu, 1998, Phys. Rev. Lett. 81, 5358.

Tang, X. P., U. Geyer, R. Busch, W. L. Johnson, and Y. Wu, 1999, Nature (London) 402, 160.

Tanner, L. E., and R. Ray, 1977, Scr. Metall. 11, 783.

Teichler, H., 1996, Phys. Rev. Lett. 76, 62.

Teichler, H., 1997, Defect Diffus. Forum 143-147, 717.

Teichler, H., 1999, Phys. Rev. B 59, 8473.

Teichler, H., 2001, J. Non-Cryst. Solids 293, 339.

Tiwari, R. S., S. Ranganathan, and M. von Heimendahl, 1981, Z. Metallkd. 72, 563.

Tu, K. N., and T. C. Chou, 1988, Phys. Rev. Lett. 61, 1863.

Tyagi, A. K., M.-P. Macht, and V. Naundorf, 1990, Scr. Metall. Mater. 24, 2369.

Tyagi, A. K., M.-P. Macht, and V. Naundorf, 1991a, Acta Metall. Mater. 39, 609.

Tyagi, A. K., M.-P. Macht, and V. Naundorf, 1991b, J. Nucl. Mater. 179-181, 1026.

van den Beukel, A., 1991, Phys. Status Solidi A 128, 285.

van den Beukel, A., 1993, Key Eng. Mater. 81-83, 3.

van den Beukel, A., 1994, Acta Metall. Mater. 42, 1273.

van Ee, L., B. J. Thijsse, and J. Sietsma, 1998, J. Non-Cryst.

Solids 232-234, 212.

Wahnstöm, G., 1991, Phys. Rev. A 44, 3752.

Warburton, W. K., and D. Turnbull, 1975, in Diffusion in Solids, Recent Developments, edited by A. S. Nowick and J. J. Burton (Academic, New York), p. 171.

Wenwer, F., A. Gude, G. Rummel, M. Eggersmann, Th. Zumkley, N. A. Stolwijk, and H. Mehrer, 1996, Meas. Sci. Technol. 7, 632 .

Wiedenmann, A., U. Keiderling, M.-P. Macht, and H. Wollenberger, 1996, Mater. Sci. Forum 2250-227, 71.

Zener, C., 1950, Imperfections in Nearly Perfect Crystals (Wiley, New York).

Zener, C., 1951, J. Appl. Phys. 22, 372.

Zhang, T., A. Inoue, and T. Masumoto, 1991, Mater. Trans., JIM 32, 1005.

Zöllmer, V., H. Ehmler, K. Rätzke, P. Troche, and F. Faupel, 2000, Europhys. Lett. 51, 75.

Zöllmer, V., K. Rätzke, F. Faupel, A. Rehmet, and U. Geyer, 2002, Phys. Rev. B 65, 220201(R).

Zumkley, Th., M.-P. Macht, V. Naundorf, J. Rüsing, and G. Frohberg, 2000, J. Metastable Nanocryst. Mater. 8, 135.

Zumkley, Th., V. Naundorf, and M. P. Macht, 2000, Z. Metallkd. 91, 901.

Zumkley, Th., V. Naundorf, M.-P. Macht, and G. Frohberg, 2001, Scr. Mater. 45, 471. 